$$
\begin{aligned}
& \text { GANIL P g4-13 } \\
& \text { sw942 }
\end{aligned}
$$
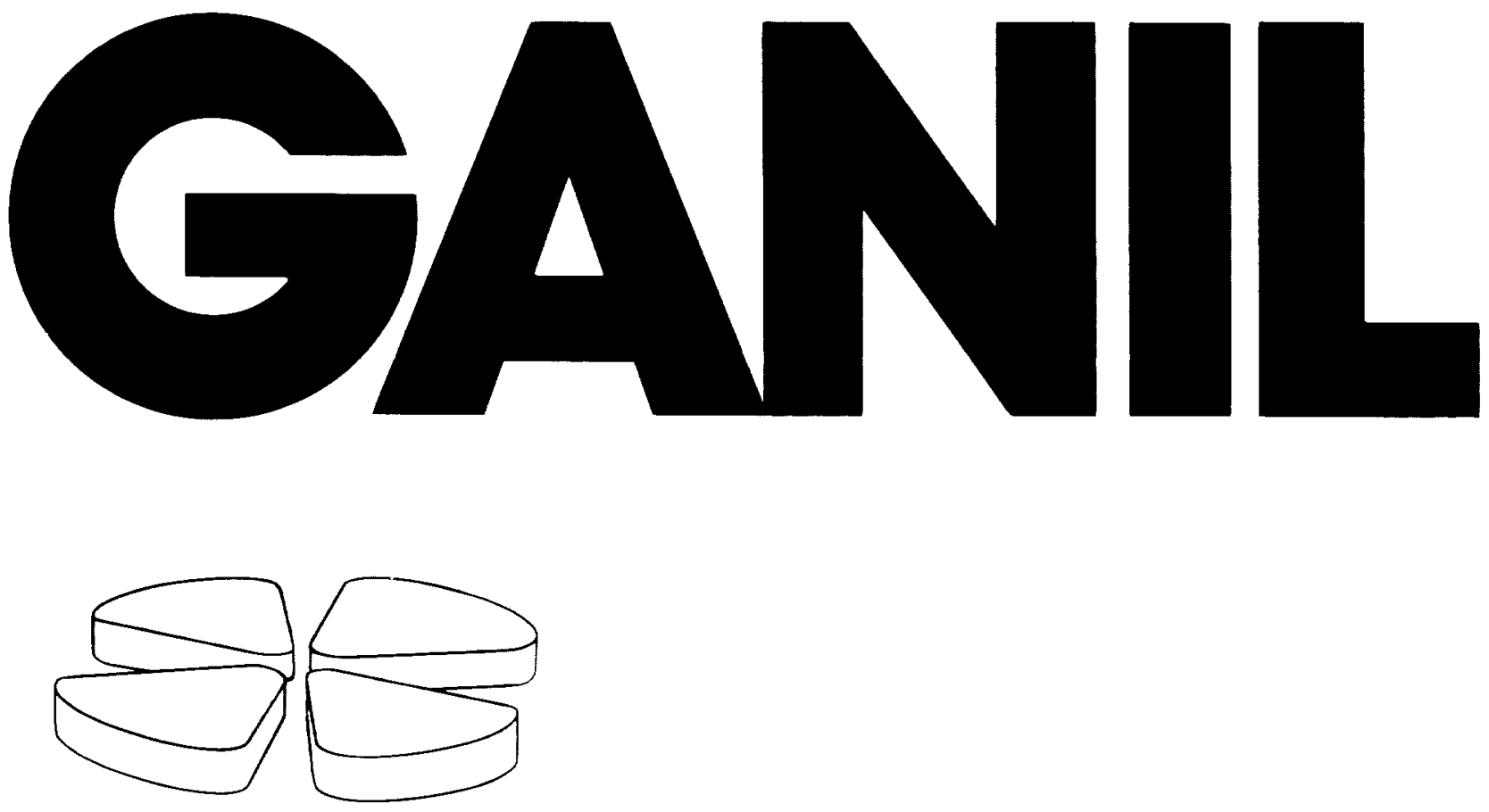

Two and many particle correlations in nnclear and high energy physics

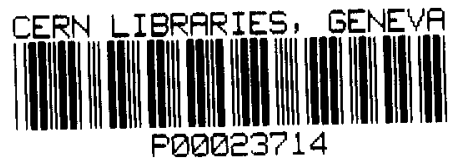

P. Bożek ${ }^{1}$, M. Płoszajczak ${ }^{2}$ and R. Botet ${ }^{3}$ 


\section{Two and many particle correlations in nuclear and high energy physics}

P. Bożek ${ }^{1}$, M. Płoszajczak ${ }^{2}$ and R. Botet ${ }^{3}$

1. H. Niewodniczański Institute of Nuclear Physics, PL-31-342 Kraków, Poland

2. Grand Accélerateur National d'Ions Lourds (GANIL), F-14021 Caen, France

3. Laboratoire de Physique des Solides, Bâtiment 510, Université Paris-Sud, Centre d'Orsay, F-91405 Orsay, France 


\section{Abstract}

The relevance of the scale-invariance and intermittency ideas for both the multiparticle production process and the nuclear multifragmentation process is disscussed. The studies of the multiparticle distributions using the correlation functions, the scaled factorial moments and the correlation integral are described. Several theoretical models of high energy multiparticle collisions and multifragmentation having the scale-invariance properties, are presented and possible theoretical ideas leading to the scaling features in these processes are explained. We present also a short résumé of the experimental status in this domain of research. 


\section{Contents}

1 Abstract 2

2 Correlation functions and factorial moments 5

2.1 Multiparticle distribution and correlation functions . . . . . . . . 5

2.1.1 Generalities and variables ............... 5

2.1.2 Distribution and correlation functions . . . . . . . . . . 6

2.1.3 Bose-Einstein correlations .............. 7

2.1.4 The cumulant distributions ............. 8

2.1.5 Moments of the multiplicity distribution . . . . . . . . . 9

2.2 Scaled factorial moments and cumulants . . . . . . . . . . . 10

2.2.1 Scaled factorial moments . . . . . . . . . . . . . 10

2.2.2 Correcting for the shape of the one-particle distribution and the

lack of the translational invariance . . . . . . . . . . . 12

2.2.3 Scaled factorial correlators, bin-split moments . . . . . . . . 14

2.2.4 Scaled factorial cumulants and correlators .......... 15

2.2 .5 Correlation integral . . . . . . . . . . . . . 16

3 Scaling correlations in nuclear and high energy physics 19

3.1 General phenomenology and experimental results ........... 20

3.2 Cascade models . . . . . . . . . . . . . . . . . . . . . 23

3.3 Phase transition . . . . . . . . . . . . . . . . . . 24

3.4 Problems occurring when analyzing the scaling behaviour . . . . . . 26

3.4.1 Nonsingular parts in the correlations . . . . . . . . . . 26

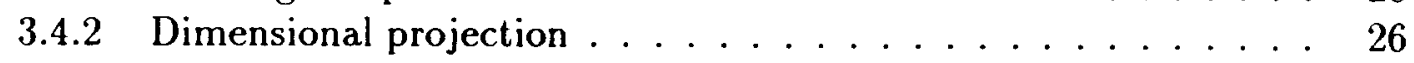

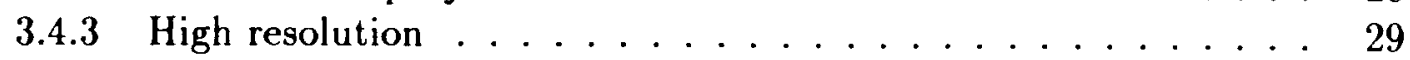

3.4.4 Effect of some cut-off . . . . . . . . . . . . . . 30

3.4 .5 Choice of the variable(s) . . . . . . . . . . . . 35

3.4.6 Event mixing in a simple model of fragmentation . . . . . . 36

4 Models $\quad \mathbf{4 1}$

4.1 Phase transition . . . . . . . . . . . . . . . . . . 41

4.1.1 High energy phase transition ............. 41

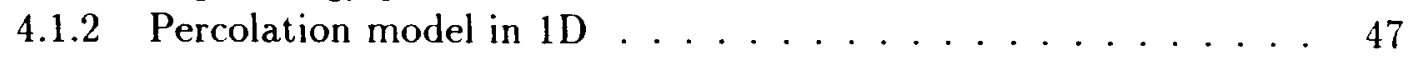

4.1.3 Percolation model in $3 \mathrm{D} \ldots \ldots \ldots 50$

4.2 Cascade models . . . . . . . . . . . . . . . . 52

4.2 .1 QCD cascade ....................... 52 
1.2.2 lragmentation-inactivation binary model . . . . . . . 5t

1.3 Bose-Einstein correlations . . . . . . . . . . . . . . . . . . . . . .

1.3.1 Suall-scale Bose-Einstein correlations . . . . . . . . . 5s

4.3 .2 Large-scale Bose-Einstein correlations. . . . . . . . . 60

4.3.3 Bose- Einstein correlations and intermittency. . . . . . . . 61

5 Closing discussion $\quad 62$ 


\section{Introduction}

The multiplicity distributions of the produced particles were studied in the high energy collisions, in the hope of learning about the production mechanism. Very early it was realized that the multiplicity distributions and, in partcular, the moments of the multiplicity distributions are related to the correlations between the produced particles. If the particles are produced independently of each other then the multiplicity distribution is poissonian. On the contrary, if the production of a particle enhances the probability to produce other particles, then the multiplicity distribution is broader than the poissonian distribution, and its scaled factorial moments (SFMs) are larger than 1. The same type of question can be asked not only for the total multiplicity distribution but also for the multiplicity of particles produced in limited cells of the phase space. This leads to the study of the correlations between particles depending on their distance. In particular, one can study by the method of the factorial moments the correlations between particles at very small scales.

It was conjectured that the multiplicity fluctuations in small phase-space bins can reveal important aspects of the multiparticle production mechanism such as the intermittent pattern of fluctuations $[1,2]$. Intermittency is characterized by large nonstatistical fluctuations at all scales, i.e. the SFMs $F_{i}$ of a studied distribution rise like $F_{i}(\delta y) \propto(\delta y)^{-\nu_{i}}$ with decreasing bin size $\delta y$ (for the definitions see sect. 2.1). Even though the underlying physical interpretation in the spirit of some multifractal structures in the multiparticle distributions is experimentally not clear, the method of the SFMs in limited rapidity intervals, proposed by Bialas and Peschanski, became a powerful tool in the studies of nonstatistical fluctuations in the multiparticle production[1] .

The two basic results of the work of Bialas and Peschanski were the proposition of applying SFMs in order to reduce the statistical noise and the study of the SFMs with changing resolution scale. The SFMs reduce the statistical noise which is present in the events with a finite multiplicity, and they remove it exactly in the case of the poissonian noise. Thus, this method permits to study effects of the nonstatistical, dynamical fluctuations in the probability distributions of produced particles without the bias from the statistical fluctuations. The dependence of the SFMs on the resolution scale in rapidity, azimuthal angle, transverse momentum or any combination of these variables was proposed as a tool to search for fractal probability distributions in multiparticle production.

The method of the SFMs is a beautiful method to investigate the multiparticle correlations on small scales and/or for high order correlations which otherwise would be inaccessible. The SFMs have also the advantage of selecting spikes in the particle distributions. The SFM of rank $i$ has contributions only from bins with at least $i$ 
particles, so higher moments are sensitive to the clusters of particles well collimated in momentum.

An important tool in these studies is the $\alpha$-model of density fluctuations [3] which was introduced in the high energy phenomenology by Biatas and Peschanski $[1,2]$. This model was used to study the different questions of the intermittency analysis, giving predictions in agreement with the experimental results.

The study of correlation at small scales is now performed by the method of the correlation integral [4]. This method allows to measure the correlations at small scales with lower errors than the SFM method. This is particularly important in the studies of the 3D correlations. Moreover, the method of correlation integral is especially suited for the study of the two-particle correlations in the square of their four-momentum $Q^{2}$ or in their invariant mass $M_{i n v}$.

In the phenomenological description of the intermittent behaviour two main approaches can be separated. The first one is the self-similar cascade picture of the multiparticle production process. The celebrated $\alpha$-model is the basic example of such mechanism. This view of the mechanism responsible for the scale-invariant correlations received recently its motivation in the perturbative QCD cascade calculations (see ref. [5] and discussion in sect. 4.2.1). Indeed, if neglecting both variation of the running coupling constant and the presence of an infrared cut-off, the QCD cascade is scale-invariant and leads to strong correlations between emitted partons. The second possible mechanism leading to the scale-invariant fluctuations is the second-order phase transition. This scheme was discussed in relation to a possible deconfimement phase transition in ultrarelativistic nuclear collisions and liquid-gas phase transistion in intermediate energy nuclear collisions. A similar mechanis could be at the origin of the fluctuations in the early universe.

In relation to these studies, it was realized that small scale Bose-Einstein correlations are important. The experimental observations indicate that this type of correlations is very strong at small scales and has power-law dependence on $Q^{2}$. This phenomenon implies the presence of a large coherent source produced even in the collisions of elementary particles.

The idea of studying the multiplicity fluctuations in small bins, could be relevant in other domains in physics. Indeed, the concept of intermittency was introduced long time ago in a theory of a classical turbulent flow [6]. The multifractal structure of the support of the intermittent measure which corresponds to the velocity field in turbulence, is geometrically clear. It is by now understood, that the spatial regions, where a hierarchy of turbulent structures appears and in which energy is transferred from larger to smaller scales, is fractal. The fragmentation, which is omnipresent in many different areas of physics, is another natural candidate to look for scale-invariance. A fragmentation process produces clusters of different sizes following collision or some other method of excitation and subsequent deexcitation. Of interest in this process is the size distribution of clusters. In certain processes, including nuclear heavy-ion collision, percolation, condensation phenomenon and many others, the power-law appears in the average cluster-size distribution. The studies of the correlations in the fragmentation have much less tradition, even though they could be of great interest, in view of non-uniqueness of the fragmentation mechanism.

Recently, it was proposed to apply the intermittency analysis for the studies of 
fragment charge distribution lollowing the decay of a hot nuclear residum of the heavy-ion collision at intermediate energies. The standard theoretical tools used to describe this process include the thermodynamical description, equilibrium and nonequilibrium binary sequential processes, percolation etc. . These different models are expected to be characterized by different fluctuations and hence, one hopes to select a correct fragmentation mechanism by looking for fluctuation properties at small scales in cluster-size distribution.

This paper contains a review of present status of theoretical studies on intermittency and correlations with a particular emphasis on the multiparticle production in different high energy collisions and on the nuclear multifragmentation phenomenon in intermediate energy heavy-ion collisions. In sect. 2 we introduce the reader to the features of the correlation functions, factorial moments, factorial cumulants etc. which can be applied to evidence the scale-invariant features of the studied distribution and, hence, the physical process of interest. The characteristic properties of each of this method will be given and connection between them outlined. The first part of sect. 3 is devoted to a short résumé of the experimental evidence for the scale invariance in high energy multiparticle collisions and in the nuclear multifragmentation process. In the second part of sect. 3, we discuss shortly the two types of models: the cascade and the phase transition models, which are generally applied in search for power-laws. We also discuss the difficulties and problems in unambiguous assessement of the scaling behaviour. Sect. 4 is devoted to the more detailed dicussion of various theoretical models and theoretical concepts related to the scaling features of the multiplicity distributions. Finally, the closing discussion is given in sect. 5 . 


\section{Correlation functions and factorial moments}

This chapter gives the essential definitions for the multiparticle distributions and correlations. The correlation observables are very important in the present studies of the high energy collisions and nuclear collisions at intermediate energies. The recently proposed methods of studying these correlations, i.e. the SFM made possible a considerable progress in this field. The methods to study these distributions at small scales, i.e. the SFM and the correlation integral are presented and summarized in this chapter.

\subsection{Multiparticle distribution and correlation func- tions}

\subsubsection{Generalities and variables}

We shall be interested in the studies of the distribution of particles or fragments produced in collisions of elementary particles or nuclei. Most frequently the correlation studies are performed for one particle specie, should it be charged particles, pions, kaons etc. It is straightforward to generalize it for the case of several particle species, such as the opposite charge particle correlations or flavour correlations.

The total inelastic cross section $\sigma_{I}$ can be written as a sum of the cross sections $\sigma_{n}$ for the production of exactly $n$-particles in an event :

$$
\sigma_{I}=\sum_{n=0}^{\infty} \sigma_{n}
$$

Furthermore, one can define the probabilities $P_{n}=\sigma_{n} / \sigma_{I}$ of observing $n$-particles in an inelastic event.

In order to study the distributions of particles in phase space, one has first to define probabilities of observing particles at given points in the phase space. Generally, the "position" (momentum) variable can be one or many dimensional. Most often the variables are the rapidity, azimuthal angle and the transverse momentum or any combination of these. For the definition of the longitudinal and transverse variables it is often required to perform a rotation, so that the longitudinal variables are defined by the sphericity or thrust axis and not by the beam axis. This change removes from the fluctuations studied, those due to different orientation of the event axis with respect to the beam axis. The invariant $Q^{2}$ distance between particles and the mass difference for the fragments distribution can also be used as the variable in which the multiparticle distributions are studied. 


\subsubsection{Distribution and correlation functions}

The one-particle distribution is the probability of observing a particle at a given point $y$ irrespective of the number and positions of other particles :

$$
\rho_{1}\left(y_{1}\right)=\frac{1}{\sigma_{l}} \frac{d \sigma}{d y_{1}} .
$$

An experimental estimator of the one-particle distribution is defined as :

$$
\rho_{1}\left(y_{1}\right)=<\sum_{k} \delta\left(x_{k}-y_{1}\right)>
$$

where $x_{k}$ are the positions of the observed particles and the brackets mean the average over the events. Mostly the experimental resolution induces some minimal binning in the measured variables. Then, the information accessible to the observer is the distribution integrated over some phase space cell $\omega_{i}$ of the size of the experimental resolution :

$$
\rho_{\omega_{i}}=\frac{1}{\omega_{i}} \int_{\omega_{i}} \rho_{1}(x) d x
$$

This is due to the fact that in a given event one only knows the number of observed particles in each of the phase space cells of minimal size. This leads naturally to the study of the multiplicity distributions and their moments in different phase space cells (sect. 2.2 ). Also in the size distribution of the fragments in the nuclear multifragmentation, we study the distribution of the number of fragments of different charge.

New method of correlation integral has been proposed [4], that gives information about integrated correlations without the artificial binning which is present in the SFM method. The results obtained using this method are then smoother and have lower experimental error bars than the factorial moments in different bins (sect. 2.2.5). The correlation integral allows also for the direct use of the $Q^{2}$ distance as a variable in the definition of the multiparticle correlations.

The information about $n$-particle correlations is contained in the $n$-particle distribution function $\rho_{n}\left(y_{1}, \ldots, y_{n}\right)$. This quantity denotes the probability density of observing $n$-particles with momenta $y_{1}, \ldots, y_{n}$ irrespective of the number and positions of any other particles. These distribution densities are related to the $n$-particle inclusive cross sections :

$$
\rho_{n}\left(y_{1}, \ldots, y_{n}\right)=\frac{1}{\sigma_{l}} \frac{d^{n} \sigma}{d y_{1} \ldots d y_{n}} .
$$

The estimator of the $n$-particle distribution is :

$$
\rho_{n}\left(y_{1}, \ldots, y_{n}\right)=<\sum_{k_{1} \neq \ldots \neq k_{n}} \delta\left(y_{1}-x_{k_{1}}\right) \ldots \delta\left(y_{n}-x_{k_{n}}\right)>.
$$

The $n$-particle distribution can be written using the $n$-particle correlation function :

$$
\rho_{n}\left(y_{1}, \ldots, y_{n}\right)=\rho_{1}\left(y_{1}\right) \ldots \rho_{1}\left(y_{n}\right)+C_{n}\left(y_{1}, \ldots, y_{n}\right),
$$

and one can define the reduced correlation function :

$$
c_{n}\left(y_{1}, \ldots, y_{n}\right)=\frac{C_{n}\left(y_{1}, \ldots, y_{n}\right)}{\rho_{1}\left(y_{1}\right) \ldots \rho_{1}\left(y_{n}\right)} .
$$




\subsubsection{Bose-Einstein correlations}

A large amount of data exists for the two-particle correlation function (2. This correlation function was studied for like-sign particles in order to extract information on the size of the enitting region from the Bose-Einstein correlations [7]. The photon interferometry was first proposed by Hanbury-Brown and Twiss [8] as a way to measure the spatial extension of extragalactic sources. The interference of undistinguishable particles was proposed as a measure the spatio-temporal extension of the interaction region in elementary particles or nuclear collisions [9]. The two-particle distribution :

$$
D_{2}\left(k_{1}, k_{2}\right)=\frac{\rho_{2}\left(k_{1}, k_{2}\right)}{\rho_{1}\left(k_{1}\right) \rho_{1}\left(k_{2}\right)}
$$

is related to the density of the emitting sources by

$$
D_{2}\left(k_{1}, k_{2}\right)=1+\left|d_{1}\left(k_{1}-k_{2}\right)\right|^{2},
$$

where $d_{1}(k)$ is the four-dimensional Fourier transform of the density of the sources. The experimentally measured like-sign pion correlation function, can be used to study the radius of the interaction region in the collision. For a gaussian source density profile, the distribution function is related to the radius of the radiating region $R$ :

$$
D_{2}(k)=1+\exp \left(-R^{2} k^{2} / 2\right),
$$

where $k \equiv k_{1}-k_{2}$. The derivation of the above equation assumes that the sources emit coherently and are randomly distributed over the sphere of radius $R$. Many works deal with the calculation of the Bose-Einstein correlation depending on the dynamic evolution and the shape of the source and different chaoticity characteristics [10]. In section 4.3.2 we shall discuss the implication of spatio-temporal correlations of the emitting sources on the Bose-Einstein correlations.

Obviously, it is very difficult to measure the full $6 \mathrm{D}$ two-particle correlation function. In the case of the spherically symmetric static source, the two-particle correlation function depends only on the value of the momentum difference between the particles. If one is interested also in measuring the lifetime of the source, then it is useful to measure also the correlation functions in different domains of the energy difference of the two bosons. However, generally one can have more complicated dependence on the momentum, an example of this is the Bjorken-scaling source, which leads to different type of correlations in longitudinal and transverse variables [11]. Other scenarios lead to $Q^{2}=-\left(p_{1}-p_{2}\right)^{2}$ as a one dimensional variable for which the two-particle correlations should be studied, e.g. in the string decay [12]. If one is looking for a new behaviour in the Bose-Einstein correlations, one is interested in some general covariant features universal to different type of collisions. Hence, the natural variable for the exploratory studies of the small scale correlations should be $Q^{2}$. The $Q^{2}$ variable can be used also as a distance in defining the many particle correlations [13]. Then one should simply measure the $n$-particle cumulant in the variable being the sum of the $Q^{2}$ of all the pairs of particles. 


\subsubsection{The cumulant distributions}

The $n$-particle correlation function consists mainly of statistical combination of lower order correlations. In order to study genuine $n$-particle correlations, one has to define the $n$-particle cumulant $K_{n}\left(y_{1}, \ldots, y_{n}\right)$, which enters into the expression for $\rho_{n}$ together with cumulants of the order lower than $n$. The first few densities are :

$$
\rho_{2}\left(y_{1}, y_{2}\right)=\rho_{1}\left(y_{1}\right) \rho_{1}\left(y_{2}\right)+K_{2}\left(y_{1}, y_{2}\right)
$$

i.e. $K_{2}=C_{2}$,

$$
\begin{aligned}
\rho_{3}\left(y_{1}, y_{2}, y_{3}\right)= & \rho_{1}\left(y_{1}\right) \rho_{1}\left(y_{2}\right) \rho_{1}\left(y_{3}\right)+\sum \rho_{1}\left(y_{i}\right) K_{2}\left(y_{j}, y_{k}\right)+K_{3}\left(y_{1}, y_{2}, y_{3}\right) \\
\rho_{4}\left(y_{1}, y_{2}, y_{3}, y_{4}\right)= & \rho_{1}\left(y_{1}\right) \rho_{1}\left(y_{2}\right) \rho_{1}\left(y_{3}\right) \rho_{1}\left(y_{4}\right)+\sum \rho_{1}\left(y_{i}\right) \rho_{1}\left(y_{j}\right) K_{2}\left(y_{k}, y_{l}\right) \\
& +\sum \rho_{1}\left(y_{i}\right) K_{3}\left(y_{j}, y_{k}, y_{l}\right)+\sum K_{2}\left(y_{i}, y_{j}\right) K_{2}\left(y_{k}, y_{l}\right)+K_{4}\left(y_{1}, y_{2}, y_{3}, y_{4}\right) \\
\rho_{5}\left(y_{1}, \ldots, y_{5}\right)= & \rho_{1}\left(y_{1}\right) \rho_{1}\left(y_{2}\right) \rho_{1}\left(y_{3}\right) \rho_{1}\left(y_{4}\right) \rho_{1}\left(y_{5}\right)+\sum \rho_{1}\left(y_{i}\right) \rho_{1}\left(y_{j}\right) \rho_{1}\left(y_{k}\right) K_{2}\left(y_{l}, y_{m}\right) \\
& +\sum \rho_{1}\left(y_{i}\right) \rho_{1}\left(y_{j}\right) K_{3}\left(y_{k}, y_{l}, y_{m}\right)+\sum \rho_{1}\left(y_{i}\right) K_{2}\left(y_{j}, y_{k}\right) K_{2}\left(y_{l}, y_{m}\right) \\
& +\sum \rho_{1}\left(y_{i}\right) K_{4}\left(y_{j}, y_{k}, y_{l}, y_{m}\right)+\sum K_{2}\left(y_{i}, y_{j}\right) K_{3}\left(y_{k}, y_{l}, y_{m}\right) \\
& +K_{5}\left(y_{1}, \ldots, y_{5}\right)
\end{aligned}
$$

where the sums are taken over all permutations of $\left\{y_{1}, \ldots, y_{n}\right\}$ without the transposition inside the factors of the sums.

The $n$-particle cumulant measures the statistical dependence of the whole $n$-particle set. The $n$-particle cumulant is zero, if anyone of the $n$ particles is independent of the others. The second order cumulant is equivalent to the two-particle correlations, but at the three (and higher) particle level the study of the cumulant distribution requires the subtraction from three-particle correlation function of the combinatorial part from the lower order correlations.

Some authors have addressed also the problem of the structure of higher order cumulants. One of the observations was that in the nuclear collisions the cumulants of order 3 and higher are zero in the experimental data [14]. For other types of reactions the linked-pair approximations of the higher order cumulants, inspired by the structure of the galaxy distribution in the universe, was applied to fit the data[14, 15]. Also another type of higher order cumulants was used giving a negative binomial multiplicity distributions in phase-space cells [16]. Both these approaches, which in fact give numerically very similar results, were found to reproduce the data for the SFM in rapidity in many reactions. However the data for $e^{+} e^{-}$annihilation does not follow this scheme, and a multiplicative structure of the higher order cumulants was used to fit the data, leading to log-normal multiplicity distributions in small bins [17]. Indeed, the multiplicity distributions in small rapidity bins for the $e^{+} e^{-}$reactions, are closer to the log-normal distribution than to the negative binomial distribution. This departure from the negative binomial form of the multiplicity distributions was confirmed in different reactions when going to small subcells in $3 \mathrm{D}[18]$. 


\subsubsection{Moments of the multiplicity distribution}

From the production probabilities one can construct the moments of the nultiplicity distribution. The $i$-th moment is given as :

$$
<n^{i}>=\sum_{n=1}^{\infty} n^{i} P_{n}
$$

Similarly, the scaled moment of the multiplicity distribution is :

$$
C_{i}=\frac{\left\langle n^{i}\right\rangle}{<n\rangle^{i}}
$$

The scaled moments $C_{i}$ are frequently used to compare multiplicity distributions for different processes in restricted rapidity intervals or for different energies, i.e. for distributions with different $\langle n\rangle$. In particular the energy independence of $C_{i}$ was expected to be a consequence of the KNO scaling of the multiplicity distributions. As we shall see below, the SFMs are a better tool to study the multiplicity distributions, because they are not contaminated by the statistical noise. Actually, they deconvolute the observed multiplicity distributions from the Poisson distribution, which is a natural ansat $z$ for statistical noise superimposed on top of the "physical" distribution. The factorial moment is defined as :

$$
<n(n-1) \ldots(n-i+1)>=\sum_{n=1}^{\infty} n(n-1) \ldots(n-i+1) P_{n},
$$

and the SFM as :

$$
F_{i}=\frac{<n(n-1) \ldots(n-i+1)>}{<n>^{i}} .
$$

Let us write the discrete multiplicity distribution $P_{n}$ in form of a Poisson transform (see ref. [19] for a discussion of the Poisson transform in the context of high energy phenomenology) :

$$
P_{n}=\int_{0}^{\infty} f(x) \frac{(x \bar{n})^{n} e^{-x \bar{n}}}{n !} d x
$$

where $\bar{n}=<n>$ and $f(x)$ fulfills the normalization conditions :

$$
\begin{gathered}
\int_{0}^{\infty} f(x) d x=1 \\
\int_{0}^{\infty} x f(x) d x=1 .
\end{gathered}
$$

The Poisson transform means a convolution of the statistical poissonian noise of mean $\bar{n}$ with the "physical" distribution $f(x)$. The SFMs of the discrete distribution $P_{n}$ are related to the moments of the function $f(x)$ :

$$
F_{i}=\int_{0}^{\infty} x^{i} f(x) d x
$$

This analysis was used to study the multiplicity distributions in the full phase-space or in some restricted rapidity windows. Some bin to bin correlations and, in particular the forward-backward correlations were also studied [19]. 


\subsection{Scaled factorial moments and cumulants}

\subsubsection{Scaled factorial moments}

The studies of the multiplicity distribution cannot show the structure of the correlations between the momenta of the produced particles. In the independent particle production, the probability of producing a particle does not depend on the fact whether and how many other particles are produced. If, on the contrary, some correlations are present, the production of $i$ particles enhance the probability of the production of the $(i+1)$-st particle. As a result, the multiplicity distributions are broader than the Poisson distribution. This gives us information on the global number of the particles produced, i.e. on the integrated correlation functions and not on the correlations between particles with definite momenta.

The integration of the $n$-particle distribution over a domain $\Omega$ of the phase-space, gives us the factorial moments of the multiplicity distribution in that domain [20] :

$$
\begin{aligned}
<n>_{\Omega} & =\int_{\Omega} d y \rho_{1}(y) \\
\ldots & =\ldots, \\
<n(n-1) \ldots(n-i+1)>_{\Omega} & =\int_{\Omega} d y_{1} \ldots \int_{\Omega} d y_{i} \rho_{i}\left(y_{1}, \ldots, y_{i}\right),
\end{aligned}
$$

and correspondingly the SFM :

$$
F_{i}=\frac{\int_{\Omega} d y_{1} \ldots \int_{\Omega} d y_{i} \rho_{i}\left(y_{1}, \ldots, y_{i}\right)}{\left(\int_{\Omega} d y \rho_{1}(y)\right)^{i}}
$$

If the one-particle inclusive distribution is approximately constant, i.e. in the "plateau" region, we can rewrite the above relation as :

$$
F_{i}=\frac{1}{\Omega^{i}} \int_{\Omega} d y_{1} \ldots \int_{\Omega} d y_{i} d_{i}\left(y_{1}, \ldots, y_{i}\right),
$$

where the $d_{i}$ represents the $i$-particle reduced density :

$$
d_{i}\left(y_{1}, \ldots, y_{i}\right)=\frac{\rho_{i}\left(y_{1}, \ldots, y_{i}\right)}{\rho_{1}\left(y_{1}\right) \ldots \rho_{1}\left(y_{i}\right)} .
$$

The use of the reduced density is very common in phenomenological parametrizations of the $i$-particle distribution in the cases, where the one-particle distributions are factorizing. In the model comparisons, we shall mostly assume furthermore that the one-particle density is almost constant.

Bialas and Peschanski proposed to study the dependence of the SFMs on the resolution in the rapidity [1]. The idea was to study the structure of the particle density in rapidity in the high energy event. They showed that the SFMs averaged over $M$ bins of width $\delta y$ in the total rapidity window of length $\Delta Y=M \delta y$, correspond to the moments of the probability density in these bins :

$$
\int_{0}^{\infty} d x_{1} \ldots \int_{0}^{\infty} d x_{M} P\left(x_{1}, \ldots, x_{M}\right) \frac{1}{M}\left(\sum_{k=1}^{M} x_{k}^{i}\right) \sim \frac{M^{i-1} \sum_{k=1}^{M} n_{k} \ldots\left(n_{k}-i+1\right)}{N(N-1) \ldots(N-i+1)},
$$


where $n_{k}$ is the mumber of particles in the bin $k$ in the event of the total multiplicity $A$ in the whole rapidity interval $\Delta Y$. An average of the right-hand side of the above equation over a large number of events with fixed multiplicity $N$, should converge to the left-hand side The multiplicity distribution in different bins $P_{m u l}\left(n_{1}, \ldots, n_{M}\right)$ is a convolution of the probability distribution $P$ and the multinomial distribution :

$$
P_{m u l}\left(n_{1}, \ldots n_{M}\right)=\frac{N !}{n_{1} ! \ldots n_{M} ! M^{N}} \int_{0}^{\infty} d x_{1} \ldots \int_{0}^{\infty} d x_{M} P\left(x_{1}, \ldots, x_{M}\right) x_{1}^{n_{1}} \ldots x_{M}^{n_{M f}}
$$

Thus, the factorial moments of the multiplicity distribution in different bins give the moments of the probability distribution $P$. The intermittent behaviour ${ }^{1}$ is defined as a power-law dependence of the SFMs on the number of bins :

$$
F_{i} \sim(M)^{\nu_{1}}=\left(\frac{\Delta Y}{\delta y}\right)^{\nu_{1}} \sim(\delta y)^{-\nu_{1}}
$$

where $\nu_{i}$ is called the intermittency exponent of rank $i$. This analysis was applied to the JACEE event [1], confirming the observation of Takagi [21] that the rapidity density fluctuations are of a non-statistical origin. The SFMs can also be averaged for a sample of many events. This allows to perform similar studies also in low multiplicity events such as discussed in $e^{+} e^{-}$or hadron-hadron collisions $[1,2]$.

The SFM is calculated for each event in a definite binning, i.e. for each event the sum over all the $M$ bins is taken, and then the average over all events is taken. Generally, the events in the sample have different multiplicities so the normalization $N(N-1) \ldots(N-i+1)$ in eq. (2.25), which accounts for the Bernoulli character of the statistical fluctuations around the studied probability $P$, is replaced by $\langle N\rangle^{i}$, where $\langle N\rangle$ is the mean multiplicity in the sample of events. This gives the horizontally averaged SFMs [2] :

$$
\left.F_{i}=\frac{M^{i-1}}{\langle N\rangle^{i}} \sum_{k=1}^{M}<n_{k}\left(n_{k}-1\right) \ldots\left(n_{k}-i+1\right)\right\rangle,
$$

where $\langle\ldots\rangle$ means an average over events.

The horizontally averaged SFM measure also the fluctuation of the total multiplicity. Now $F_{i}(1) \neq 1$ and gives the moments of the total multiplicity. These fluctuations arise from the integration of the correlations over the whole phase-space. It is expected, at least in the rapidity variable, that the correlations have two parts: a long range part describing the total multiplicity correlations and a short range part describing small scale behaviour of the production process [22]. Thus, the two-particle reduced density can be written as :

$$
d_{2}(\Delta p)=1+C_{l}(\Delta p)+C_{s}(\Delta p),
$$

where the long range correlation are a smooth function for $\Delta p \rightarrow 0$, e.g. $C \exp (-\Delta p / \xi)$ and the short range correlations can have very strong dependence for small scales, e.g.

\footnotetext{
${ }^{1}$ We shall use the name intermittency signal or intermittency pattern to describe the increase of the SFMs with the resolution, but not necessarily a power-law. We shall also call an intermittency signal a stronger one, if the corresponding local slopes of the dependence of the SFM on the resolution are bigger
} 
the power-law $\left({ }_{s}(\Delta p)=\left({ }^{\prime}(\Delta p)^{-1}\right.\right.$. It is expected that the short range power-law behaviour can be extracted by fitting to the SFil the form [2:3] :

$$
F_{2}(\omega)=1+B+C^{\prime} \omega^{-\prime_{2}} .
$$

Note, however that it is not a priori obvious that the short range correlations describe mainly the correlations induced by production process and are well separated from the long range correlations describing the mixing of events of different multiplicities, e.g. due to the mixing of different impact parameters in the event sample. Generally, if events in different classes have very different one-particle distribution, then mixing of different types of events could induce strong short range correlations, even though, each particular class of events does not show any increase of the fluctuations at small scales. An example could be the mixing of two types of events characterized by two exponential transverse momentum distributions with different slope parameters. Even though, each class of events has no correlations, and all events are similar to the particular average in one of the classes, the average of the mixed sample is very different from any typical event of a particular class. The whole sample would show fluctuations, which do not describe the fluctuations occurring in any production process but which are due to the mixing of different types of events, each with no fluctuations. This problem shows that in order to understand the structure of the correlations it is important to measure the SFMs in separate bins, without bin averaging, and also some bin to bin correlations such as the scaled factorial correlators (sect. 2.2.3). This question will be discussed more in details in the nuclear fragmentation case (sect. 3.4.6), where it is known to be a serious problem.

\subsubsection{Correcting for the shape of the one-particle distribution and the lack of the translational invariance}

Generally, even if the particles are uncorrelated (all correlation functions $C_{n}=0$ ), the SFM defined above, contains a spurious dependence on the scale due to the shape of the one-particle distribution $\rho_{1}(y)$. So the horizontal SFMs should be corrected for this dependence by a factor [24] :

$$
\left.R_{i}=\frac{M^{i-1}}{\langle N\rangle^{i}} \sum_{k=1}^{M}<n_{k}\right\rangle^{i} .
$$

The corrected SFMs :

$$
\tilde{F}_{i}=\frac{F_{i}}{R_{i}},
$$

are less biased by the variations in the single-particle spectrum. Ochs, Bialas and Gaździcki proposed to look at the fluctuations in a different variable [25]:

$$
X(y)=\frac{\int_{y_{\operatorname{man}}}^{y} d y^{\prime} \rho_{1}\left(y^{\prime}\right)}{\int_{y_{\operatorname{man}}}^{y_{\max }} d y^{\prime} \rho_{1}\left(y^{\prime}\right)},
$$

where $0 \leq X(y) \leq 1$. This change of variables before the intermittency analysis is especially important for rapidly changing $\rho_{1}$, such as for the transverse momentum 
distribution. Where this procedure was shown to better follow the true intermitent correlation than the corrected horizontal analysis. The experimental data are almost entirely analyzed using the corrected, horizontally averaged SFMs $\dot{F}_{2}$. The SFMs calculated in that way for each chosen bin width are then fitted to the intermittent relation :

$$
\ln \left(\grave{F}_{i}\right) \sim a-\nu_{i} \ln (\delta y)
$$

In the following, if not said explicitly, we shall use the notation $F_{1}$ for the SFMs corrected for the shape of the one-particle distribution.

One can also define the vertically averaged SFMs $[2,26]$ :

$$
F_{i}=\frac{1}{M} \sum_{k=1}^{M} \frac{\left.<n_{k}\left(n_{k}-1\right) \ldots\left(n_{k}-i+1\right)\right\rangle}{\left.<n_{k}\right\rangle^{i}} .
$$

This corresponds to the average of the SFMs calculated in each of the $M$ bins over all the bins. This form of averaging is equivalent to the previous one for the case of the flat one-particle distribution. However, there are till now not many experimental data on the vertical SFMs. The EMU01 data for the nuclear collisions [27], show little difference between the vertical SFMs and the corrected horizontal SFMs.

From the theoretical point of view, it is often easier to relate the vertical SFMs to the integrals of the $n$-particle distributions :

$$
F_{i}(\delta y)=\frac{1}{M} \sum_{k=1}^{M} \frac{\int_{y_{\min }+(k-1) \delta y}^{y_{\min }+k \delta y} d y_{1} \ldots \int_{y_{\min }+(k-1) \delta y}^{y_{\min }+k \delta y} d y_{i} \rho_{i}\left(y_{1}, \ldots, y_{i}\right)}{\left(\int_{y_{\min }+(k-1) \delta y}^{y_{\min }+k \delta y} \rho_{1}(y)\right)^{i}} .
$$

For slowly varying $\rho_{1}(y)$, SFMs can be written using the reduced densities as follows :

$$
F_{i}(\delta y)=\frac{1}{M} \sum_{k=1}^{M} \frac{1}{(\delta y)^{i}} \int_{y_{\min }+(k-1) \delta y}^{y_{\min }+k \delta y} d y_{1} \ldots \int_{y_{\min }+(k-1) \delta y}^{y_{\min }+k \delta y} d y_{i} d_{i}\left(y_{1}, \ldots, y_{i}\right) .
$$

Generally, one assumes translational invariance of the reduced densities and consequently, of the SFMs in different bins. The reduced densities depend then only on the relative variables $y_{i}-y_{k}$, so that one has:

$$
F_{i}(\delta y)=\frac{1}{(\delta y)^{i}} \int_{0}^{\delta y} d y_{1} \ldots \int_{0}^{\delta y} d y_{i} d_{i}\left(y_{1}, \ldots, y_{i}\right)
$$

The comparison of the horizontal SFMs to the phenomenological parametrization of the reduced density is difficult because the contribution of different bins is weighted by the single-particle distribution $\rho_{1}(y)$ for that bin. The vertical moments have the contribution of each bin scaled so that all of them enter on equal footing to the sum over the bins. For sufficiently small bins they are closer to the integrals of the reduced density (eq. 2.38) than to the horizontal moments even if the one-particle distribution is not flat. In the next sections we shall extensively use the relation (2.38) between the SFMs and the reduced densities, assuming a simple and approximately translationally invariant parametrization for the reduced densities. 


\subsubsection{Scaled factorial correlators, bin-split moments}

The SFM in a rapidity window $\delta y$ is equivalent to the $n$-particle distribution function integrated from the scale 0 to $\delta y$ (eq. 2.38). Thus, $F_{i}(\delta y)$ has contributions from the $i$-particle distribution on all this range of scales. This makes difficult to disentangle the true scaling behaviour of the multiparticle distributions in a certain range of rapidity separations from other effects, which could be present at the limiting scales. This concerns especially the behaviour of the $n$-particle distribution for particle separation going to 0 . The presence of some limit on the scaling behaviour in this region, can change dramatically the dependence of the integrated $n$-particle distribution on the upper integration limit $\delta y$ (see $[28,29]$ and sect. 3.4.4). On the other hand, the limit $\delta y \rightarrow 0$ tests only a limited part of the $i$-particle phase-space $\left(\left|y_{i}-y_{k}\right|<\delta y \rightarrow 0\right)$. To cure this disadvantage of the SFMs, Bialas and Peschanski proposed to study the scaled factorial correlators (SFCs) which are the observables relating the fluctuations in separated bins [2]. The SFC $F_{i, j}$ for two bins of width $\delta y$ separated by the distance $D$ is defined as :

$$
F_{i, j}(\delta y, D)=\frac{\left.<n_{1}\left(n_{1}-1\right) \ldots\left(n_{1}-i+1\right) n_{2}\left(n_{2}-1\right) \ldots\left(n_{2}-j+1\right)\right\rangle}{\left\langle n_{1}\left(n_{1}-1\right) \ldots\left(n_{1}-i+1\right)><n_{2}\left(n_{2}-1\right) \ldots\left(n_{2}-j+1\right)\right\rangle},
$$

where $n_{1(2)}$ is the number of particles in the first (second) bin. SFC defined in this way, is then averaged over all the pairs of bins distant by $D$ in the rapidity window $\Delta Y$, what corresponds to the vertical averaging. In was shown in the $\alpha$-model that the SFCs are independent of the bin size $\delta y$ and exhibit a power-law dependence on the bin distance D :

$$
F_{i, j} \sim(D)^{-\nu_{i, j}} .
$$

$\nu_{i, j}$ in the above expression is called the intermittency exponent of the SFC $F_{i, j}$. The SFCs can also be directly related to the integrals of the $(i+j)$-particle distribution function :

$$
\begin{aligned}
& F_{i, j}(\delta y, D)=\frac{1}{M} \sum_{k=1}^{M}\left(\int_{y_{\min }+(k-1) \delta y}^{y_{\operatorname{men}}+k \delta_{y}} d y_{1} \ldots \int_{y_{\min }+(k-1) \delta y}^{y_{\min }+k \delta y} d y_{i}\right. \\
& \left.\int_{y_{m: n}+D+(k-1) \delta_{y}}^{y_{\min }+D+k \delta_{y}} d y_{i+1} \ldots \int_{y_{\min }+D+(k-1) \delta_{y}}^{y_{\min }+D+k \delta_{y}} d y_{i+j} \rho_{i+j}\left(y_{1}, \ldots, y_{i+j}\right)\right) / \\
& \left(\int_{y_{\min }+(k-1) \delta y}^{y_{\min }+k \delta y} d y_{1} \ldots \int_{y_{\min }+(k-1) \delta y}^{y_{\min }+k \delta y} d y_{i} \rho_{i}\left(y_{1}, \ldots, y_{i}\right)\right. \\
& \left.\int_{y_{m i n}+D+(k-1) \delta y}^{y_{\min }+D+k \delta y} d y_{1} \ldots \int_{y_{\min }+D+(k-1) \delta y}^{y_{\min }+D+k \delta y} d y_{j} \rho_{j}\left(y_{1}, \ldots, y_{j}\right)\right) \text {, }
\end{aligned}
$$

where $M=(\Delta Y-D) / \delta y$ is the number of the bin pairs in the rapidity window $\left[y_{\min }, y_{\min }+\Delta Y\right]$. For slowly varying single-particle density, the SFCs can be approximated by simple integrals of the $(i+j)$-particle reduced distributions. This form will be used in the further investigations.

Moments similar to the SFC's have also been proposed by Seibert and Voloshin [30]. They proposed the split-bin correlators, i.e. the bin of width $\delta y$ is divided in two parts 
and the correlations in the left and right part of the bin are calculated:

$$
F_{2}^{(S B)}=\frac{1}{M} \sum_{k=1}^{M} \frac{\left\langle n_{L} n_{R}\right\rangle}{\left.\left\langle n_{L}\right\rangle<n_{R}\right\rangle} .
$$

This definition corresponds to the SFC: $F_{1,1}$ for the case $\delta y=D$. Due to the approximate independence of the SFCs on the bin width $\delta y$ both in the $\alpha$-model [2] and in the experiment [31], SFCs and the split-bin correlators are largely equivalent.

The advantage of the split-bin moments is that they allow the analysis of the fluctuations in continuum observables such as the transverse energy in certain subdomains of the rapidity and/or azimuthal angle. Dealing with the small scale correlations in the case of a thermodynamic model, Haglin and Seibert [32] made an interesting observation of the difference in the results for the SFMs and the split-bin moments. The split-bin correlation are sensitive to the scale on which they are calculated and are not contaminated at all scales by the zero scale behaviour. This could be important if the experimental data are contaminated by some double track counting, which introduces spurious correlations at the scale of the resolution of the detectors. The analysis by the split-bin moments or the SFCs could disentangle these spurious correlations from other nontrivial correlation at other scales.

The SFCs and SFMs are all related to the $n$-particle distribution function and so there exist relations between them [33]. These relations are true for any type of translationally invariant distributions. In the case of self-similar distributions, one obtains relations also between the intermittency exponents of the SFMs and SFCs :

$$
\nu_{i, j}=\nu_{i+j}-\nu_{i}-\nu_{j}
$$

\subsubsection{Scaled factorial cumulants and correlators}

The $n$-particle distribution is the sum of the trivial product of the single-particle distributions and of the $n$-particle correlation functions. Generally, one studies the behaviour of the SFMs which tests the whole $n$-particle distribution function. Selfsimilar behaviour in this variable is expected in the $\alpha$-model, but generally it is not clear whether the self-similar behaviour is present in the scaled factorial moments, in the scaled factorial cumulants or whether the power-law correlations are only a part of all the different correlations in the multiparticle distributions. The scaled factorial cumulants have the advantage of testing the genuine $n$-particle correlations, and so it is always interesting to test their contribution to the higher order correlation for each process.

The cumulant correlation functions can be studied using the scaled factorial cumulants :

$$
\mathcal{K}_{i}=\frac{\int_{\Omega} d y_{1} \ldots \int_{\Omega} d y_{i} K_{i}\left(y_{1}, \ldots, y_{i}\right)}{\left(\int_{\Omega} d y \rho_{1}(y)\right)^{i}}
$$

If the one-particle inclusive distribution is approximately constant, i.e. in the "plateau" region, we can rewrite the above relation as :

$$
\mathcal{K}_{i}=\frac{1}{\Omega^{i}} \int_{\Omega} d y_{1} \ldots \int_{\Omega} d y_{i} k_{i}\left(y_{1}, \ldots, y_{i}\right),
$$


where the hi represents the i-particle reduced cumblant:

$$
h_{1}\left(y_{1}, \ldots, y_{2}\right)=\frac{h_{i}\left(y_{1} \ldots y_{i}\right)}{\rho_{1}\left(y_{1}\right) \ldots \rho_{1}\left(y_{i}\right)}
$$

Obviously, in this case a relation exists between the scaled factorial moments and the scaled factorial cumulant analogous to the relations for the non-integrated quantities (2.13) :

$$
\begin{aligned}
& F_{2}=1+\mathcal{K}_{2} \\
& F_{3}=1+3 \mathcal{K}_{2}+\mathcal{K}_{3} \\
& F_{4}=1+6 \mathcal{K}_{2}+4 \mathcal{K}_{3}+6 \mathcal{K}_{2} \mathcal{K}_{2}+\mathcal{K}_{4} \\
& F_{5}=1+10 \mathcal{K}_{2}+10 \mathcal{K}_{3}+30 \mathcal{K}_{2} \mathcal{K}_{2}+5 \mathcal{K}_{4}+5 \mathcal{K}_{2} \mathcal{K}_{3}+\mathcal{K}_{5}
\end{aligned}
$$

In the case when the multiparticle densities are not translationally invariant, the above relations, except for the first one, are only approximate. Thus, it is required to study the scaled factorial cumulants directly from the experimental data.

Analogously one can define the cumulant correlators, obtained by the integration of cumulant distributions in separated bins :

$$
\begin{aligned}
\mathcal{K}_{i, j}(\delta y, D)= & \frac{1}{M} \sum_{k=1}^{M}\left(\int_{y_{\min }+(k-1) \delta y}^{y_{m+n}+k \delta y} d y_{1} \ldots \int_{y_{\min }+(k-1) \delta y}^{y_{\min }+k \delta y} d y_{i}\right. \\
& \left.\int_{y_{\min }+D+(k-1) \delta y}^{y_{\min }+D+k \delta y} d y_{i+1} \ldots \int_{y_{\min }+D+(k-1) \delta y}^{y_{\min }+D+k \delta y} d y_{i+j} K_{i+j}\left(y_{1}, \ldots, y_{i+j}\right)\right) / \\
& \left(\int_{y_{\min }+(k-1) \delta y}^{y_{\min }+k \delta y} d y_{1} \ldots \int_{y_{\min }+(k-1) \delta y}^{y_{\min }+k \delta y} d y_{i} \rho_{i}\left(y_{1}, \ldots, y_{i}\right)\right. \\
& \left.\int_{y_{\min }+D+(k-1) \delta y}^{y_{\min }+D+k \delta y} d y_{1} \ldots \int_{y_{\min }+D+(k-1) \delta y}^{y_{\min }+D+k \delta y} d y_{j} \rho_{j}\left(y_{1}, \ldots, y_{j}\right)\right)
\end{aligned}
$$

where $M=(\Delta Y-D) / \delta y$ is the number of the bin pairs in the rapidity window $\left[y_{\min }, y_{\min }+\Delta Y\right]$.

More generally, Fialkowski proposed to analyze the scaled factorial moments excluding not only the trivial correlations occurring from the combinatorial correlations of uncorrelated particles but also some possible long range component in the correlations [23]. This leads to the formula $(2.30)$ for the intermittency component in the second SFM.

\subsubsection{Correlation integral}

The method of SFMs use definite binning in the phase-space which induces strong fluctuations when changing the scale. Due to the statistical errors, this limits the lowest scales accessible by this method. Another method to measure the correlations in phase-space cells is the correlation integral method[4]. The SFM are the integrals of the many-particle densities over some phase-space cell. The vertically or horizontally 
averaged SFll ate given as averages of such integrats over phase-space cells of size $\Omega$. If it happens that several particles lie in the same cell. then the SFM have a large value. However. it could happen that particles are close to each other but in two or more neighbouring cells. Thus SFM have strong fhetuations in their values depending on whether it happens that a cluster of correlated particles is in one, arbitrarily chosen phase-space cell or not. The correlation integral gives a solution to this drawback by choosing the integration domain only by the condition (for the second moment) $\left|p_{1}-p_{2}\right|<\delta y$ and that both particles lie in the observed phase-space (fig. 1). Then, the correlation integral of the second order takes the form :

$$
\mathcal{C}_{2}=\frac{\int_{\Omega_{s}} d p_{1} d p_{2} \rho_{2}\left(p_{1}, p_{2}\right)}{\int_{\Omega_{3}} d p_{1} d p_{2} \rho_{1}\left(p_{1}\right) \rho_{1}\left(p_{2}\right)},
$$

where $\Omega_{s}$ is the strip (or in higher dimension a tube) as in fig. 1 . One can see that the integration region is smoother than for the SFM. This makes the result more stable and the statistical errors are smaller. To define the tube, especially in higher dimension, one has to decide what is the distance in momentum between two particles. This can be the usual distance : $\sqrt{\left(y_{1}-y_{2}\right)^{2}+\left(\phi_{1}-\phi_{2}\right)^{2}+\left(p_{T_{1}}-p_{T_{2}}\right)^{2}}$, or the maximum of the distance in each variable. It can be also the four momentum square distance. According to the chosen distance one defines the tube $\Omega_{s}$ of size $\delta y$, as the region where any interparticle distance in the cluster is smaller than $\delta y$. Obviously, for orders higher than two this requires also a definition of the topology of the cluster, i.e. the definitions of all the distances in the cluster that are taken into account. Several topologies were proposed $[4,34]$ :

1. the Grassberger - Henschel - Procaccia (GHP) topology, where the size of an nparticle cluster is given by the maximum of all the $n(n-1) / 2$ distances between any particle in the cluster (fig. $2 \mathrm{a}$ ),

2. the snake topology, where one calculates the maximum of the $n-1$ distances between the first and the second, the second and the third particle and so on (fig. $2 b)$,

3. the star cluster, where one takes as the size of the cluster the maximum of the $n-1$ distances of all the particle of the cluster from one chosen particle in the cluster (fig. 2c).

According to this, the $n$-particle correlation integral can take the following form :

$$
\begin{aligned}
\mathcal{C}_{n}(\delta y)= & \left(\int_{\text {phasespace }} d p_{1} \ldots d p_{n} \rho_{n}\left(p_{1}, \ldots, p_{n}\right) \Theta\left(\delta y-\left|p_{1}-p_{2}\right|\right) \ldots \Theta\left(\delta y-\left|p_{1}-p_{n}\right|\right)\right. \\
& \left.\Theta\left(\delta y-\left|p_{2}-p_{3}\right|\right) \ldots \Theta\left(\delta y-\left|p_{2}-p_{n}\right|\right) \ldots \Theta\left(\delta y-\left|p_{n-1}-p_{n}\right|\right)\right) \\
& /\left(\int_{\text {phasespace }} d p_{1} \ldots d p_{n} \rho_{1}\left(p_{1}\right) \ldots \rho_{1}\left(p_{n}\right) \Theta\left(\delta y-\left|p_{1}-p_{2}\right|\right) \ldots \Theta\left(\delta y-\left|p_{1}-p_{n}\right|\right)\right. \\
& \left.\Theta\left(\delta y-\left|p_{2}-p_{3}\right|\right) \ldots \Theta\left(\delta y-\left|p_{2}-p_{n}\right|\right) \ldots \Theta\left(\delta y-\left|p_{n-1}-p_{n}\right|\right)\right),
\end{aligned}
$$


for the (illp topology,

$$
\begin{aligned}
\mathcal{C}_{n}(\delta y)= & \left(\int_{p h a s t s p a c t} d p_{1} \ldots d p_{n} \rho_{n}\left(p_{1}, \ldots, p_{n}\right) \Theta\left(\delta y-\left|p_{1}-p_{2}\right|\right) \Theta\left(\delta y-\left|p_{2}-p_{3}\right|\right) \ldots\right. \\
& \left.\Theta\left(\delta y-\left|p_{n-1}-p_{n}\right|\right)\right) \\
& /\left(\int_{\text {phasespace }} d p_{1} \ldots d p_{n} \rho_{1}\left(p_{1}\right) \ldots \rho_{1}\left(p_{n}\right) \Theta\left(\delta y-\left|p_{1}-p_{2}\right|\right) \Theta\left(\delta y-\left|p_{2}-p_{3}\right|\right) \ldots\right. \\
& \left.\Theta\left(\delta y-\left|p_{n-1}-p_{n}\right|\right)\right)
\end{aligned}
$$

for the snake topology, and

$$
\mathcal{C}_{n}(\delta y)=\frac{\int_{\text {phasespace }} d p_{1} \ldots d p_{n} \rho_{n}\left(p_{1}, \ldots, p_{n}\right) \Theta\left(\delta y-\left|p_{1}-p_{2}\right|\right) \ldots \Theta\left(\delta y-\left|p_{1}-p_{n}\right|\right)}{\int_{\text {phasespace }} d p_{1} \ldots d p_{n} \rho_{1}\left(p_{1}\right) \ldots \rho_{1}\left(p_{n}\right) \Theta\left(\delta y-\left|p_{1}-p_{2}\right|\right) \ldots \Theta\left(\delta y-\left|p_{1}-p_{n}\right|\right)},
$$

for the star cluster topology, respectively.

The star correlation integral is most often used in the experimental analysis because the computing time of the numerator in (2.52) is proportional to $N^{2}$, where $N$ is the multiplicity, in contrast to the GHP and snake integrals which require a computing time proportional to $N^{n}$ and $N^{n} / n$ ! respectively. Inserting the estimators for the multiparticle densities (eq. 2.6) in the equation for the star correlation integral, one obtains after a change of variables [34]:

$$
\mathcal{C}_{n}(\delta y)=\frac{<\sum_{i_{1}}\left(\sum_{i_{2} \neq i_{1}} \Theta\left(\delta y-\left|p_{1}-p_{2}\right|\right)^{[n-1]}>\right.}{\left\langle\sum_{i_{1}}\left(\sum_{i_{2} \neq i_{1}} \Theta\left(\delta y-\left|p_{1}^{e v_{1}}-p_{2}^{e v_{2}}\right|\right)^{n-1}\right\rangle\right.}
$$

In the numerator, the sphere count around the particle $i_{1}$ is raised to the factorial power $[n-1]$ and in the denominator to the usual power $n-1$. In the denominator we take the particles from different events by the procedure of event mixing. A simpler procedure which is often used, consists of taking the first particle from one event and inserting it into another event and then calculating the sphere counts around the inserted particle. This simple procedure introduces some bias inversely proportional to the multiplicity but is computationally faster, the cpu time required being proportional to the square of the number of events. The correlation integral can be defined also for the calculation of the cumulant integrals and for the calculation of the so called differential factorial correlations, i.e. differences of the correlation integral at two different scales $\delta y_{1}$ and $\delta y_{2}$. This corresponds to the integration of the correlations at scales between $\delta y_{1}$ and $\delta y_{2}$ similarly as in the factorial correlators. The method of the correlation integrals is now widely used in the experimental analysis, because it give results on the integrated multiparticle densities with lower errors than the SFM method (sect. 3.1). 


\section{Scaling correlations in nuclear and high energy physics}

The original proposal to look for the scale-invariance in the multiparticle distributions $[1,2]$, was very appealing. The idea besides its simple form (no specific scale involved) made reference to the well known scale-invariant and fractal structures occurring in many different physical systems. Such scale-invariant systems are characterized by the scaling exponents $\nu$ which often are universal, 1.e. they do not depend on the specific details of models or physical systems if these are in the same universality class. This feature is characteristic for the second order phase transitions but also for same multifractal models. This possibility could give new insight in the theory of the multiparticle production. Another possibility was related to the self-similar cascade which is a common picture of the multiparticle production. This inspired the simple $\alpha$-model (sec. 3.2), recently revived by the studies of the QCD cascade (see sect. 4.2.1). Another mechanism which could generate scaling behaviour, independently of the type and energy of the reaction, could be the self-organized criticality, which without fine tuning of the parameters, like in the phase transition point, leads to the scaling distributions.

Recently, it was proposed to apply the intermittency analysis to the studies of the fragment charge distribution following the decay of hot nuclear residuum $[35,36]$. In the nuclear collisions at incident energies $E / A \leq 1 \mathrm{GeV}$, the particle production is strongly suppressed and the nuclear breakup into fragments dominates. At low excitation energies, nucleus breaks into one or possibly two heavy fragments and a number of light evaporated particles. At somewhat higher excitation energies $\left(4 \mathrm{MeV} \leq E^{*} \leq\right.$ $6 \mathrm{MeV}$ ), the nuclear residuum may break into a larger number of medium size fragments. This phenomenon is called the multifragmentation process. Finally, at high energies, the collision leads to a complete disintegration of the colliding nuclei into separate nucleons and light nuclear fragments $\left({ }^{2} \mathrm{H},{ }^{3} \mathrm{H},{ }^{4} \mathrm{He}, ..\right)$. Nuclei are many body systems that are large enough for the concepts of statistical mechanics to be applicable with some confidence. On the other hand, they are still small enough so that one can expect fluctuations to be very important. In certain situations, the fluctuations are much more important than expected from the finiteness of the system and dominate the average characteristics of the system, such as happens in a turbulent motion of a fluid [6]. The discovery of intermittency in nuclear multifragmentation could be interpreted in a natural way as an evidence for self-similarity and scaling in the decompression phase of the evolution, preceding the fragmentation of a hot residue in a high energy collision between heavy ions. If this interpretation is correct, it will most likely have important consequences for the theory of the dynamics of heavy-ion collisions. Several authors, drawing the analogy between the distribution of cluster sizes at the percolation threshold [37] or the droplet sizes at the critical point [38] and the distribution of nuclear fragments in mass and charge [39] suggested the possibility of a liquid-gas phase transition in finite nuclear systems. A more quantitative examination of this hypothesis is due to Campi [40], who proposed an event by event analysis of the nuclear multifragmentation using the method of conditional moments and showed 
that nuclei break up as a fuite percolating network. (ertainly. the percolation model simplifies the physical picture of nuclear breakup. However, it contains the two most essential elements of the fragmentation process, i.e. the locality of the breaking process involved in the cluster formation and the short range character of the nucleon-nucleon interaction. The possibility of a critical behaviour associated with percolation transition was one of the motivations for looking for the appearance of strong fluctuations and intermittency in nuclear multifragmentation.

In this context, it is interesting to mention that intermittency has been also reported in low energy, ion induced collision cascades in solids [41]. The energy fluctuations, extracted from analyzing atom kinetic energy distributions, exhibit anomalous dimensions that are several times larger than the maximum anomalous dimensions observed so far in nuclear reactions both at intermeciiate and high energies[42] .

\subsection{General phenomenology and experimental re- sults}

The experimental data for the fragment charge distribution in the nuclear multifragmentation are not very numerous and come essentially from interactions in nuclear emulsion. First data analyzed using the SFM method [35] correspond to the breakup of ${ }_{79}^{197} A u_{118}$ nuclei of energy $E / A \simeq 1 \mathrm{GeV}$ in a nuclear emulsion [43]. The measured multiplicities of fragments vary strongly from event to event, ranging over different dynamical situations of collision and of a subsequent break up. The dependence of the SFMs $F_{i}$ on the bin size $\delta s$, where for $s$ we take the charge of the fragments $(1 \leq s \leq 79)$, is shown in Fig. 3 . On the left-hand side of Fig. 3, a sample of events with the fragment multiplicity $28<\tilde{M}<56 \quad(0.35<m<0.7$ in the scaled units $m \equiv \tilde{M} / \tilde{M}_{\max }$, where $\tilde{M}_{\max }=79$ is the maximal possible size (三 charge) of the fragment). On the right-hand side of Fig. 3, events with a large number of heavy fragments $N_{f r} \geq 3$ of charge $s_{f r} \geq 3$ are shown. In both cases the linear growth of SFMs clearly manifests the intermittent pattern of fluctuations in the charge distribution. It should be noted, that the values of moments and the intermittency slopes depend on the selection criteria for the events. These data could be qualitatively reproduced by the 3D percolation model (see Fig. 23 in sect. 4.1.3) $[35,36,44]$. Recently, similar features have been reported in the nuclear interactions induced from ${ }_{92}^{238} U_{146}$ nuclei at approximately the same incident energy [45].

The experimental results on the SFM and correlation integral analysis and on the short correlations measurements in the high energy collisions are presented in a recent review by De Wolf et al. [48]. In this sections we shall only list some most important observations of the large experimental survey in this domain.

The lowest beam energies used in studies of non-statistical fluctuations in the spectra of particles produced in nuclear reactions are $1.52 \mathrm{GeV} / \mathrm{A}[46]$ and $2.1 \mathrm{GeV} / \mathrm{A}$ [47] In these experiments, the $1 \mathrm{D}$ and $2 \mathrm{D}$ phase-space distributions of produced charged hadrons, analyzed using the SFM method, exhibit an intermittent behaviour. The relativistic hadronic transport model has been applied for studies of pion multiplicity distributions and fluctuations in charged pion pseudorapidity distributions in this beam energy region [49]. It was found that the pion pseudorapidity distributions show inter- 
mittency with intermittency slopes strongly depending on the beam energy [49, 50]. It was then, suggested that the appropriate variable to study intermittency in this energy domain is the kinetic energy of pions. In this variable. the intermittency slopes are independent of the bombarding energy [50] .

Different experimental groups analyzed the correlations between particles produced in high energy collisions. First the $1 \mathrm{D}$ and $2 \mathrm{D}$ data were analyzed in the SFM method. Also the $3 \mathrm{D}$ data were recently studied, but the main results of this analysis are obtained using the correlation integral method.

The $e^{+} e^{-}$annihilation was first analyzed by the TASSO Collaboration [51]. The analysis was performed in the $1 \mathrm{D}$ rapidity $(y)$ distribution along the sphericity axis and in the $2 \mathrm{D}$ rapidity-azimuthal angle $(y-\phi)$ distributions. At LEP energies, DELPHI [52], ALEPH [53] and OPAL [54] Collaborations performed the intermittency analysis in 1D. 2D or 3D distributions. Fig. 4 shows the results of the DELPHI Collaboration on the intermittency analysis of the particles produced in the $c^{+} e^{-}$annihilation. The second factorial moment for the $1 \mathrm{D}$ analysis in rapidity, 2D analysis in rapidity-azimuthal angle, and the full 3D analysis are shown. The effect of the dimensional projection, which reduces the 3D correlations if observed $1 \mathrm{D}$ or $2 \mathrm{D}$, is clearly visible. In this analysis, the LUND model predictions were found to be consistent with the data. The CELLO Collaboration analyzed the 3D intermittency signal in $e^{+} e^{-}$annihilation and also found a good agreement with the LUND model [55]. A characteristic feature of the $3 \mathrm{D}$ data is the strong correlation between the like-charge particles. These correlations are much stronger than those between the unlike-charge particles[56]. This means that the two and many particle correlations are mainly due to the Bose-Einstein interference mechanism. However, some part of the correlations in 3D was identified as due to the resonance decay and cascade structure of the production mechanism. The charge correlations in $1 \mathrm{D}$ and $2 \mathrm{D}$, look very much the same, but this could be due to the strong effect of the dimensional projection.

The SFM analysis was made also for the $\mu$-p scattering[57]. These data could not be reproduced by the LUND and Marchesini-Webber models. Unlike in the other experiments, the authors found that a significant part of the effect could be due to the Bose-Einstein correlations already in the $1 \mathrm{D}$ data. This effect, similarly as in the other data, is much more pronounced in the $3 \mathrm{D}$ results [58].

In the neutrino - nucleus interactions, an intermittency signal in $1 \mathrm{D}$ rapidity analysis was found for $\nu$-Ne interactions and no intermittency was found for $\nu$-D interactions [59]. The intermittency in this experiment was interpreted as an effect of rescattering in the $\nu$-nucleus interaction.

The $\pi^{+} / K^{+}-p$ collisions were extensively analyzed by the NA22 Collaboration [60, 61]. Fig. 5 shows the results of the NA22 Collaboration on the second SFM in the 1D, $2 \mathrm{D}$ and $3 \mathrm{D}$ analysis [48]. Like in the other reactions, a strong dimensional projection effect is observed, leading to the suppression of the fluctuations in the lower dimensional spectra.

Fig. 6 presents the results of the same group in the 3D analysis, separately for all charged, negatives and unlike-charge particles[48]. In the like-charge particles, the main effect is due to the Bose - Einstein correlations which, if included in the FRITIOF simulations, reproduce the data. On the other hand, the correlations between unlikecharge particles are due to the Dalitz pairs and the $\gamma$ conversion. As expected, in the 
sample containing all particles these effects are mixed.

The data of the UAI ('ollaboration for $p p$ collisions at 630 GeV indicated an increase of the intermittency signal for the low multiplicity samples [62]. This tendency is not reproduced by the models. The $3 \mathrm{D}$ analysis of the $p-\bar{p}$ collisions by the UAl Collaboration confirms the observation that the main part of the correlations at small scales are due to the corrclations between like-sign particles. As can be seen in Fig. 7 , the dominance of the Bose-Einstein correlations was identified in the correlation integrals up to order $5[6.3]$. The $p$ - $p$ collisions at $360 \mathrm{GeV}$ were analyzed in 1D for different multiplicity samples. The Monte-Carlo generators also do not reproduce the multiplicity dependence of the fitted intermittency slopes [64].

The NA22 Collaboration performed the intermittency analysis of the particles produced in $\pi^{+} / K^{+}$-nucleus interactions [65], using the same experimental setup as for the $\pi^{+} / K^{+}-p$ collisions studied earlier by this group $[60,61,31]$. The results show weaker intermittency signal for larger targets. Generally, the 3D correlations seem to be of the Bose-Einstein type, and have a power-component in the two-particle correlations [63]. Of course, the signal is always reduced in the $1 \mathrm{D}$ and $2 \mathrm{D}$ analysis by the dimensional projection effect.

The proton-nucleus and nucleus-nucleus collisions were first analyzed by the KLM Collaboration $[66,67]$ in $1 \mathrm{D}$ and 2D distributions. The intermittency signal decreased for larger projectiles, but this decrease was smaller than expected from the increase of the mean multiplicity in the collision. It was impossible to reproduce this nonlinear dependence on the multiplicity by the models of independent collisions. The EMU01 Collaboration performed also the intermittency analysis for different nuclear projectiles and targets $[27,68]$ and found a similar dependence as the KLM Collaboration. Generally, the intermittency signal decreases rapidly with increasing incident energy $(14.6,60$ and $200 \mathrm{GeV})$.

The NA35 Collaboration analyzed the multiparticle production in the nuclear collisions also in the 3D momentum space [69]. In Fig. 8, the results for the correlation integral of like-sign and unlike-sign particles in different reactions are shown. Once more the correlations are much stronger between the like-sign particles. The strength of the correlations does not decrease as the inverse of the mean multiplicity of the reaction [69], indicating that we deal with a collective effect such as the Bose-Einstein correlations. 1D and 2D analysis of the nuclear collisions was also performed for emulsion experiments at different energies [70]. Few events from cosmic ray experiments were analyzed finding rather strong intermittency effect [71].

In summary, all studied high energy processes show an increase of fluctuations with increasing resolution. However, the SFMs dependence on the bin size flattens for small bins in $1 \mathrm{D}$ analysis. An intermittency signal was found stronger in $2 \mathrm{D}$ and even stronger in 3D analysis, where no sign of flattening is seen. The rise of the factorial moments at small scales could be parametrized by a singular power-law component of the twoparticle correlation function [23]. Moreover, it is clear that the strong correlations at small scales are present in the samples of the like-charge particles. This leads to the conclusion that the quantum interference gives the main contribution to the observed strong correlations.

The $e^{+} e^{-}$annihilation results are consistent with the predictions of the LUND model. The hadron-hadron collisions show however an opposite dependence of the 
intermittency signal on the muliplicity cuts as the FRITlOF model. In the nuelear and hadron collisions. the Monte-('arlo calculations fail to reproduce the strength of the intemittency signal. This is only possible by the jnclusion of the Bose-Einstein correlation in the Monte-('arlo results which then become consistent with the data $[18,72]$.

\subsection{Cascade models}

As we mentioned already, the simplest model where the self-similar density fluctuations occur is the self-similar cascade model. Those type of models were first introduced in the description of the energy dissipation in the fully developed turbulence [6]. The main ideas behind this model are the independent evolution of different branches of the cascade with increasing resolution and the random multiplicative nature of the density fluctuations.

The $\alpha$-model was introduced by Bialas and Peschanski [2], as a model of multiparticle production in high energy collisions. In this section, we recall the definitions and the basic results of the $\alpha$-model of the multiparticle production. The model predicts fluctuations of the rapidity density generated by a random multiplicative process. The random cascade is self-similar and thus gives scale-invariant rapidity density fluctuations. At each step of the cascade all the rapidity subintervals are subdivided into $\lambda$ parts. ( See Fig. 9, where four steps of the cascade are shown for $\lambda=2$.) At each subdivision the density in the resulting rapidity interval is multiplied by a random variable $W$. So that after $n$ steps the density in an interval of length $l=\Delta Y / \lambda^{n}$ is given by a product of $n$ independent random variables $W_{1}, \ldots, W_{n}$ :

$$
\rho_{m}=W_{1} \ldots W_{n} .
$$

Due to the self-similarity of the cascade all these variables have the same probability distribution $P(W)$. The variables are defined so that :

$$
<W^{\prime}>\int P(W) W d W=1 .
$$

The moments of the density $\rho_{m}$ in the $m$-th bin have a power-law dependence on the resolution $l$ :

$$
<\rho_{m}^{i}>\sim\left(\frac{\Delta Y}{l}\right)^{\nu_{1}}
$$

where $\nu_{i}$ are the intermittency exponents :

$$
\nu_{\imath}=\frac{\left.\ln <W^{i}\right\rangle}{\ln \lambda} .
$$

This leads to a power-law behaviour of the SFMs of the multiplicity distributions obtained by a superposition of the poissonian noise on top of the fluctuations of $\rho$. The formula (3.4) is valid for the case of the weak intermittency [28] $\nu_{i}<i-1$. Otherwise one obtains the limiting exponents of the strong intermittency $\nu_{i}=i-1$. The exponents $\tau_{\imath}$ are related to the Renyi fractal dimensions :

$$
D_{i}=\frac{\tau_{i}}{1-i} \text {. }
$$


The relations between the intermittency indices and the Renyi dimensions are well known and were used in the $f(\alpha)$ singularity spectrum analysis of the random cascade model [7:3].

The SFes can also be defined for the o-model [2] :

$$
F_{i, j}^{m, m^{\prime}}=\frac{\left.<\rho_{m}^{i} \rho_{m^{\prime}}^{j}\right\rangle}{\left.<\rho_{m}^{i}><\rho_{m^{\prime}}^{j}\right\rangle}
$$

The SFC's for the $\alpha$-model are independent on the width of the bins $m$ and $m$ ' and have a power-law dependence on the distance $D$ between the two bins :

$$
F_{i, j} \sim\left(\frac{\Delta Y}{D}\right)^{\nu_{i, j}}
$$

The exponents $\nu_{i, j}$ are related to the exponents of the SFMs :

$$
\nu_{i, j}=\nu_{i+j}-\nu_{i}-\nu_{j}
$$

Different approximations allow to calculate the exponents for the $\alpha$-model. In the gaussian approximation we have $\nu_{i} \sim i(i-1) \nu_{2} / 2$. This approximation is very crude. It is expected to be valid only for the first few moments and, moreover, it does not fulfill the constraint of the weak intermittency. The more general parametrization in terms of the Levy-stable laws has also been proposed [74]. It is straightforward to construct experimental estimates of the moments of the density $\rho$ for the cases of the poissonian statistical noise, the translational invariant correlations and for the flat one-particle distribution [2].

In the sect. 4.1.1 we shall use another model of the energy density fluctuations, the so-called $p$-model, which is a simplified version of the $\alpha$-model with $\lambda=2$. This model proposed for the description of the classical turbulence was compared to the high energy intermittency phenomena by Buschbeck and Lipa [75]. The probability distribution in the $p$-model is given by :

$$
P(W)=\frac{1}{2} \delta(W-p)+\frac{1}{2} \delta(W+2-p),
$$

with the restriction that the two bins created in the division of the same bin in a given step of the cascade have complementary density factors, one $p$ and the other $2-p$. This kind of "less-random" cascade has no global density fluctuations.

\subsection{Phase transition}

The concept of scaling at the critical point of a statistical system raised very early the question about the existence of nontrivial scaling fluctuations in such systems [76]. The divergence of the correlation length at the critical point leads to scale invariant correlations and gives power-law components in the dependence of the factorial moments or correlation integral on the scale. Indeed, the scale-invariant fluctuations in the vicinity of the critical point were observed in the $2 \mathrm{D}$ Ising model [76]. The SFMs were calculated for a $Z_{2}$ symmetric definition of the number of spins in a cell [77]. On 
the other side. the scaling of the number of spins in a cell of a given size was shown to be related to the critical index $y$ of the lsing model [TS]. It was also noticed that the statistical noise in the cell of $N$ spins is expected to be of the binomial type instead of the poissonian type [79], so that a slightly different definition of the SFMs was proposed:

$$
F_{i}=\frac{<n(n-1) \ldots(n-i+1)>N^{2}}{<n\rangle^{i} N(N-1) \ldots(N-i+1)} .
$$

The correct relation between the rise of the SFMs of the number of spins up in a cell and the scaling indices of the Ising model was calculated [80]. This problem was resolved for the Ising model with scaling spin correlation function and, generally, one has to find in a similar way the correct estimate of the scaling in the critical system for each particular case (SFMs, scaled faciorial cumulants, central moments, etc.).

Another feature of the critical systems is that the scaling properties are often not present in the two-field average $\left\langle\sigma(r) \sigma\left(r^{\prime}\right)\right\rangle$ but rather in the two-field cumulant $<\sigma(r) \sigma\left(r^{\prime}\right)>-<\sigma(r)><\sigma\left(r^{\prime}\right)>\sim\left|r-r^{\prime}\right|^{-\nu}$. An example of such an observable is the number of spins up in the Ising system, for which the resulting relation of the SFMs to the scaling properties of the critical Ising system are given in ref. [80]. Different definitions of the moments give very different results in finite systems. The directed percolation models exhibit scaling in the SFMs [81]. This means that the scaling in these models occurs in the two-point occupation probability or in the two-point connectivity on the percolating cluster. The problem of the correct observable to measure the scaling properties in small discrete systems has not a general solution, and in each case separately one should carefully look for the correct scaling observable.

The long distance limit in which one studies scaling in statistical systems gives, by convexity, different conditions on the scaling indices [82] :

$$
\nu_{i} / i \leq \nu_{j} / j \quad \text { for } \quad i \geq j
$$

than in the multiparticle phenomenology. The equality sign in (3.11) is true for ordered systems, like the Ising model, whereas the inequality is true for disordered systems having an infinite hierarchy of exponents like e.g. the random Ising system. In general the form of the correlations of higher order is not known. However, one expects for systems having only a finite number of critical indices, that the corresponding intermittency indices would depend linearly on the order of the moment :

$$
\nu_{i} \simeq \nu_{2} i / 2 \text {. }
$$

This observation was proposed by Bialas and $\mathrm{Hwa}$ as a signal of the phase transition in the relativistic nuclear collisions [83].

The detailed structure of the correlation functions in finite and anisotropic systems was discussed in the model of a finite conformal critical system[84]. The conclusions are that if the system is not only scale-invariant but also has the conformal invariance, then this symmetry could be observed by specific behaviour of finite systems with asymmetric boundaries [84]. Another specific test could be obtained by the studies of the higher order correlations, the structure of which is known in the conformal systems. The resulting conclusions are too schematic to be directly applied to the high energy phenomenology. This could be however an approach in studies of the detailed 
structure of the three monremtum distribution functions. In particular. such issues as the role of the $p_{T}, y$ variables and the $p_{T}$-dependence could be studied in such examples [84]. Another example which could give insight into the problem of the asymmetric correlation functions is the relation between the directed and the undirected percolation model $[85]$.

\subsection{Problems occurring when analyzing the scaling behaviour}

\subsubsection{Nonsingular parts in the correlations}

Here we shall comment on a problem which now seems to be well understood but which made a lot of discussion in the last years. This is the problem of the correct choice of the variable(s) where the scaling occurs. In the mathematical sense, the fractal dimensions and the scaling indices are always defined in the limit of the scale going to 0 . Of course, in the experiment we are limited by the resolution of the apparatus. Also in the theoretical models of physical systems, one expects the presence of some limiting scale at which the self-similarity is broken. Although for the case of weakintermittency one could in principle go with the size of the momentum scale to 0 without self-consistency problems, we know, however, that the production of physical particles is limited by some infrared cutoffs. In the experiment it is even more problematic to isolate a scaling behaviour since often we have only a limited range of scales accessible experimentally. Thus, although the nonsingular or less-singular terms are irrelevant for the mathematical limiting definition, nevertheless these terms are extremely important in the experimental analysis to define precisely the fitting form of the correlations in the limited range of finite scales. This problem was at the origin of the discussion whether one should expect the self-similarity in the cumulants or in the densities [14]. In general, any nonsingular term in the correlations is important, not only the one coming from the lower order correlations. Obviously, the two approaches give different results in the fits for the intermittency exponents [86]. Now the standard approach consist of removing the nonsingular terms and approximating their dependence on the scale by a constant. This means that the two-particle densities have not only the trivial 1 coming from the uncorrelated particles, but also they could have some long range non-singular correlations. Thus, a description of the experimental data should include both the non-singular, long-range correlations and the power-law component (sect. 2.2.1) in the cumulants[23].

\subsubsection{Dimensional projection}

It was observed by Ochs and Wosiek that the $2 \mathrm{D}$ intermittency analysis in rapidity and azimuthal angle gives a stronger intermittency signal than the $1 \mathrm{D}$ analysis in the rapidity alone [87]. This was interpreted as an effect of the pencil-jet structure in the branching model[87]. Later Ochs showed that the effect is quite general and it is to be expected that the $1 \mathrm{D}$ projected distributions have no longer scale-invariant fluctuations, even though the system possess such a structure in its full-dimensional version [88]. 
He studied in particular the dimensional projection in the o-model. Similar studies have been performed by Bialas and Seixas for a phenomenotogical model of singular multiparticle distributions in 31) momentum space [89]. They have observed that the effect of the dimensional projection depends on the range of the transverse momenta over which one averages the fluctuations. The discussion of the importance of the factorization properties of the 2I) multiparticle distributions was made in ref. [90]. The effect of the dimensional projection in space-time was studied in the model of phasetransition for the nuclear collisions. There the strength of the effect was linked with the transverse size of the interaction region [90]. The dependence of the attenuation of the intermittency signal on the transverse size of the system or on the transverse momentum cuts can be studied quantitatively [91].

Our discussion will concentrate on the second SFM, for which it is particularly easy to relate its behaviour with the singular structures of the two-particle density $\rho_{2}\left(\overrightarrow{r_{1}}, \overrightarrow{r_{2}}\right)$. For slowly varying $\rho_{1}(\vec{r})$, i.e. in the "plateau" region, one gets for the second SFM (eq. $2.23)$ :

$$
F_{2}^{(n)}(\delta \Omega)=\frac{1}{\Omega \delta \Omega} \sum_{i=1}^{M^{n}} \int_{\Omega_{1}} d \overrightarrow{r_{1}} \int_{\Omega_{t}} d \overrightarrow{r_{2}} d_{2}\left(\overrightarrow{r_{1}}, \overrightarrow{r_{2}}\right),
$$

where the $n$-dimensional volume $\Omega$ is divided in $M^{n}$ cubes $\Omega_{i}$ of volume $\delta \Omega$. The intermittent fluctuations in the density distribution should be observed as a power-law behaviour of the factorial moment :

$$
F_{2}^{(n)}(\delta \Omega) \sim(\delta \Omega)^{-\nu_{2}}
$$

This type of behaviour for small cubes $\delta \Omega$ is equivalent to the existence of singularity in the reduced density $d_{2}$. In the following we shall assume, that this singularity is dominating and hence, in the calculation of $F_{2}^{(n)}$ we shall omit all non-singular terms in $d_{2}$. This corresponds to the power-law behaviour of the SFM and not of the scaled factorial cumulant. Below, we consider translationally invariant densities with two different parametrizations of the singularity. The factorized parametrization :

$$
d_{2}^{(f a c)}\left(x_{1}, \vec{X}_{1}, x_{2}, \vec{X}_{2}\right)=\frac{C_{2}}{\left|x_{1}-x_{2}\right|^{\nu_{2}}\left|\vec{X}_{1}-\vec{X}_{2}\right|^{(n-1) \nu_{2}}},
$$

where $\overrightarrow{r_{i}} \equiv\left[x_{i}, \vec{X}_{i}\right]$, and the isotropic parametrization :

$$
d_{2}^{(i s o)}\left(\overrightarrow{r_{1}}, \overrightarrow{r_{2}}\right)=\frac{C_{2}}{\left|\overrightarrow{r_{1}}-\overrightarrow{r_{2}}\right|^{n \nu_{2}}}
$$

Results do not change if one rescales one of the components of $\vec{r}$ in (3.16), allowing in this way for non-isotropic shapes of $d_{2}$. In this case, by a suitable coordinate transformation which changes only the integration domain $\Omega$, one can bring the singularity back to the isotropic form (3.16). This latter modification is negligible for small bins, and hence the behaviour of the factorial moments is similar in these two cases. Thus, it does not change the qualitative behaviour of the SFM for the projected distributions, i.e. the flattening of the SFMs for small bins in rapidity. However, the variation of the integration region $\Omega$ changes the resulting "effective slope" in the $1 \mathrm{D}$ intermittency analysis (sect. 4.1.1) and can be analyzed by the finite-size scaling method [91]. 
Both factorized $d_{2}^{(f+n)}$ and isotropic $d_{2}^{(t s o)}$ yjes of singularities give a power-law behaviour for $F_{2}^{(n)}$ with the exponent $\nu_{2}$. However, they have a different behaviour in the $(n-1)$-dimensional subspace $\vec{x}$. The $(n-1)$-dinensional SFM can be obtained from the density $d_{2}$ by integrating over $x_{2}$ :

$$
F_{2}^{(n-1)}(\delta \omega)=\frac{1}{\Omega \delta \omega} \sum_{i=1}^{1 / n-1} \int_{\Delta_{x}} d x_{1} \int_{\Delta x} d x_{2} \int_{\omega_{1}} d \vec{X}_{1} \int_{\omega_{i}} d \vec{X}_{2} d_{2}\left(x_{1}, \vec{X}_{1} ; x_{2}, \vec{X}_{2}\right)
$$

The factorized singularity $d_{2}^{(f a c)}$ (eq. 3.15) gives a power-law dependence also in $(n-1)$-dimensions :

$$
F_{2}^{(n-1)}(\delta \omega) \sim A^{\prime}(\delta \omega)^{\nu_{2}}
$$

On the contrary, the reduced isotropic density has no more a singularity in $\vec{X}$ variables and, consequently, it does not show a power-law behaviour of the SFM. In particular, in the case $n=2$ such as for the $(y-\phi)$-distribution, the $1 \mathrm{D} \mathrm{SFM}$ in the limit $\delta X \rightarrow 0$ can be written as :

$$
F_{2}^{(1)}(\delta X)=(\delta X)^{-1} \int_{0}^{\delta X} d X d_{2}(X)
$$

where $d_{2}(X) \equiv \int_{\Delta x} d x_{1} \int_{\Delta x} d x_{2} d_{2}\left(x_{1}, x_{2}, X\right)$ and $X \equiv X_{1}-X_{2}$. The intermittency exponent at the scale $\delta X$ is given by :

$$
\nu_{2}(\delta X)=-\frac{d \ln F_{2}^{(1)}(\delta X)}{d \ln \delta X}
$$

After a substitution of (3.19) into eq. (3.20) one obtains :

$$
\nu_{2}(\delta X)=\frac{\delta X d_{2}(\delta X)}{\int_{0}^{\delta X} d X d_{2}(X)}-1 .
$$

For $\delta X \rightarrow 0$, the intermittency exponent $\nu_{2}(\delta X)$ tends to zero if the density $d_{2}(X)$ has no singularity at $X=0$. Using the similar arguments as above, one can argue that for finite bins $\delta X$ one should observe a flattening of the SFM. As an example of such a behaviour we show in Fig. 10 the dependence of $F_{2}^{(1)}$ in $1 \mathrm{D}$ on the rapidity resolution $\delta y$ for the singular density $d_{2}{ }^{(i s o)}\left(\vec{r}_{1}, \vec{r}_{2}\right) \quad\left(\vec{r}_{i} \equiv\left[y_{i}, \phi_{i}\right], \quad i=1,2\right)$ :

$$
d_{2}\left(\overrightarrow{r_{1}}, \overrightarrow{r_{2}}\right)=C_{2}(\Delta R)^{-\nu_{2}}
$$

where $\Delta R \equiv\left|\overrightarrow{r_{1}}-\overrightarrow{r_{2}}\right|$. The 1D factorial moments shown in Fig. 10, have been calculated for the singular $2 \mathrm{D}$ reduced density (3.22) with $\nu_{2}=0.15$. This value of the singularity exponent corresponds to the observed slope of $F_{2}^{(2)}$ in $(y-\phi)$ bins in the $e^{+} e^{-}$annihilation process [51]. The 1D moments show no power-law behaviour. Moreover, the behaviour of $F_{2}^{(1)}(\delta y)$ depends not only on the singularity but also on the cut-off parameter even for small rapidity bins. A similar type of behaviour in small rapidity bins can be obtained from the non-singular reduced density [22] :

$$
d_{2}\left(y_{1}-y_{2}\right)=1+\gamma \exp \left(-\left|y_{1}-y_{2}\right| / \xi\right) \text {. }
$$

An even stronger effect of the dimensional projection is observed when projecting a $3 \mathrm{D}$ singular density. In this case, the flattening of the $1 \mathrm{D} \mathrm{SFM}$ is stronger and the 
"effective" slopes are smaller than the ones resulting from a 2l) to 11) projection. The experimental results confirm that the strongest intermittency exists in the 31 ) analysis. and that the intermitent signal decreases with the mumber of the effective projections of the multiparticle distribution $[55,69,67 . \mathrm{i} 8]$. This conld be taken as an evidence for the non-factorized type of singularity in the reduced density. A similar behaviour of the correlation function has been found in the proton - emulsion data [92] : the hadron pairs with small pseudorapidity separation $|\Delta \eta|<0.2$ are correlated in the azimuthal angle, and hence $(\eta-\phi)$ - correlations do not factorize.

The discussion of different behaviour of the $\alpha$-model in different regimes was performed by Brax and Peschanski [93]. They 'ound that the three phases of the $\alpha$-model behave differently in the dimensional projection. One of such phases could explain the appearance of anomalous events such as scen by the NA22 Collaboration [94] and another one the jet structure observed in the $e^{+} e^{-}$annihilation. In the language of distribution functions, all these regimes should have different parametrization in 2D.

The dimensional projection from different transverse sizes, discussed in relation to the nuclear collision geometry (sect. 4.1.1) and to the finite size effect in a critical system [91], could be the dominant effect in the observed dependence of the intermittency signal on the $p_{T}$-cut [61], as proposed by Bialas and Seixas [89]. This would mean that the intermittency effect is not due to hard processes, since it is present and even stronger in low $p_{T}$ samples.

\subsubsection{High resolution}

The goal of the intermittency analysis of the multiparticle correlations was the study of these correlations at very small scales. The SFM method and even more the correlation integral approach, allow to go very high in the resolution. Actually, often the smallest scales analyzed are limited by the experimental resolution in momentum. Obviously one cannot analyze correlations between particles at the scales smaller than the experimental error on the measured momentum difference between these particles. Beyond this natural limitation one encounters other less trivial effects which occur due to the very high sensitivity of the SFM observables at small scales. The values of the SFM in very small bins are determined by a very small percentage of events which have clusters of particles grouped in small phase-space cells. This requires a very careful analysis of the experimental events in order to eliminate any contamination of double track counting. As it was shown $[32,95]$, even a slight contamination of the experimental event sample by this type of events determines the results for the SFM at small scales. Another effect of this kind is the photon conversion leading to correlated $e^{+} e^{-}$pairs, which are also responsible for part of the observed correlations at small scales between different charge particles [69]. At small scales one can also reach some limitations due to the insufficient statistics. Obviously, for small bins the results for the SFM have larger statistical errors, but also some systematical error can be present at small scales. This is known as the empty bin effect [95] and occurs if the statistics is too small in order to have a smooth one-particle distribution in small bins. Then, the SFM which are normalized by the one-particle distribution, show some flattening for high resolution. 


\subsubsection{Effect of some cut-off}

In a framework of the a-model, the distinction is made between the case of the strong and weak intermittency $[28,96]$. Let us consider the $\alpha$-model with the branching number $\lambda$ and a certain probability distribution function $P(w)$ of the random multiplicative factors [1]. The case of the weak intermittency corresponds to the probability distributions satisfying :

$$
<w^{i}><i-1 .
$$

In this case, the self-similar cascade can be infinite and the total multiplicity has a limit distribution [96]. If the condition (3.24) is not satisfied, then the cascade must be finite. For the rapidity intervals of length $\delta y \gg l$, where $l$ is the limiting minimal scale of the cascade, one obtains the following behaviour of the SFMs :

$$
F_{i} \sim(\delta y)^{1-i}
$$

i.e., the intermittency exponents are now $\nu_{i}=i-1$.

In the parametrization using the singular distribution function, the SFMs are related to the integrals of the reduced distribution functions (eq. 2.38). The singular scale-invariant distribution function :

$$
d_{i}\left(\lambda y_{1}, \ldots, \lambda y_{i}\right)=\lambda^{-\nu_{i}} d_{i}\left(y_{1}, \ldots, y_{i}\right)
$$

gives rise to the intermittent behaviour of the SFM :

$$
F_{i} \sim(\delta y)^{-\nu_{i}}
$$

However, this is correct only if the integral in eq. (2.38) exists. This constraint gives bounds on the values of the intermittency exponents $\nu_{i}$. Let us consider the case when all the $n$-particle distributions have the form :

$$
d_{i}\left(y_{1}, \ldots, y_{i}\right) \sim\left\{\prod_{(m)}\left|y_{k}-y_{l}\right|^{-\nu}\right\}_{s y m}
$$

where the number $m$ of the pairs $\left|y_{k}-y_{l}\right|$ is at least $i-1$ and at most $i(i-1) / 2$, and the whole expression is symmetrized over the permutations of $\left\{y_{1}, \ldots, y_{i}\right\}$. If the integral in eq. (2.38) exists, then the SFM has an intermittent behaviour with the intermittency exponent $\nu_{i}=m \nu$. For the above written distribution functions, the integral (2.38) exists if the intermittency exponents satisfy :

$$
\nu_{i}<i-1 .
$$

In this case, one obtains intermittent behaviour of the SFMs for all scales and there is no need to introduce any regularization of the singularity in the distribution function. This situation corresponds to the weak intermittency in the $\alpha$-model [96]. However, if the distribution function (eq. 3.26) shows a self-similar behaviour with the exponent $\nu_{i}>i-1$, then to ensure the existence of the integral in eq. (2.38), one should introduce some regularization for $\left|y_{k}-y_{l}\right| \rightarrow 0$. Let us suppose that if the distance between any pair of points $y_{k}, y_{l}$ is smaller than a certain cut-off value $l$, then 
$\left|y_{k}-y\right|$ will be replaced by $\mid$. For $d y \gg 1$. The sfolls have then the following behaviour :

$$
F_{1}(\delta y)=a l^{1-1-\nu_{2}}(\delta y)^{1-t}+b(\delta y)^{-\mu_{1}}+O\left(\delta y^{2}\right) .
$$

('karly, the above expression is divergent for $l \rightarrow 0$ if $i-1<\nu_{i}$. For $\delta y \gg l$, the first term is dominant giving the behaviour :

$$
F_{i}(\delta y) \sim(\delta y)^{1-i}
$$

similarly as in the a-model for the case of the strong intermittency. Here, analogously as in the $a$-model where the number of steps in the cascade must be finite, a nonsingular behaviour has to be assumed in the distribution function, i.e. a finite scale below which the scale-invariance breaks down. Similarly, we recover the scale-invariant behaviour of the SFMs (eq. 3.31) at scales $\delta y \gg l$, but with the exponents of the strong intermittency $\nu_{\mathrm{i}}=i-1$. Up to now we discussed the influence of the cut-off in the correlation function on their measure by the SFM. We have seen that in order that the SFMs behave in the same way as the correlation functions the singularity in the correlation functions must be integrable.

There is also another aspect of the presence of the cut-off in the production-process. This is a presence of some physical cut-off in the multiparticle cascade which would change the correlations and also the SFMs at some scale [97]. It is obvious that physically one expects the presence of some limiting scale beyond which the scaling breaks down.

Let us consider the case when the number of particles in a given cell of the phasespace is generated according to the Poisson distribution with the mean proportional to the probability density in this cell. Increasing the resolution, the total density is partitioned between different cells so that the mean density per cell is $1 / M$. Finally, one obtains very low energy densities in small cells. In the standard approach [2], these cells contribute to the multiparticle production by the generation of particles with a small mean multiplicity. For sufficiently small probability density this mean multiplicity can be much less than 1 . One faces then the question, whether it is physically reasonable to generate particle from such low available density (energy). In fact, if one has in mind the picture that the density in one cell is proportional to the energy available in this cell, then one does not expect particle creation if the available energy is smaller than the pion mass. Let us define the hadronization scale as a minimal energy necessary say to produce one particle in a given cell. We shall define the cutoff parameter $\epsilon$ as a ratio of the so defined hadronization scale to the total available energy in the system. If one considers the random cascading as a model of the string fragmentation, then the cut-off could correspond to the ratio of the transverse mass of the particle to the energy of the string. In the fully developed turbulence, the effect of the cut-off is known as the intermediate dissipation range which causes the viscous cut-off to switch off the scaling in the energy density moments [98]. Fluctuations in the distribution of the energy in the different phase-space cells make the effect of the cut-off visible already at resolutions larger than $\epsilon \Omega$, i.e. before the mean value of the energy per cell reaches the hadronization scale.

In the cascade picture at this limiting scale, the cascade starts to be discrete rather than continuous. In the modified cascade model, in the low density bins the particles 
are not formed with very low multiplicity by the Poisson statistical noise. These bins are assumed to be empty, i.e. no particles can be created any more in these bins (this mechanisms we shall call the discretization of the cascade). For densities above some cut-off value the Poisson distributed particles are produced with the mean multiplicity proportional to the density. Realistically, one expects that the Poisson distribution of the produced particles will be modified even for densities slightly above the threshold due to the constraint on the energy in the bin. Anyway, the multifractal structure of the self-similar random cascade model changes this sharp cut-off value of the density in a range of scales at which more and more branches of the cascade become extinguished, similarly to the intermediate dissipation range in the turbulence [98]. If this is the way by which the hadronization sets on in the random cascade, then the multiscaling fluctuations are in fact fluctuations due to the hadronization. The SFMs in small bins test the regions of the cascade which cannot be viewed as a convolution of the continuum random cascade with the discrete Poisson noise, since precisely in this region the discretization of the cascade produces the discrete Poisson distribution. Similar fluctuations can occur in the absence of the self-similar cascade. An example of this phenomenon can be the stronger fluctuations in the number of links than in the number of spins in the 1D Ising model [99]. We do not know, however, if there exists in the general case a universal, multiscaling dependence on the cut-off parameter such as eq. (3.44).

The multiscaling effect was found in the distribution of avalanches in the sand pile models [100], in the convective turbulence [101] and in the relaxation of supercooled liquids [102]. The explanation of the multiscaling effects for multifractals was given by Jensen, Paladin and Vulpiani [103]. Below we recall some of their results on the multiscaling of the $C_{i}$ moments in the multifractals.

The standard result for the multifractal set without any cut-off, is that the moments of the fractal probability density can be written using the singularity spectrum $f(\alpha)$ [104]. Let the probability density in a bin scale as $l^{\alpha}$ with its length $l=1 / M$ and let the number of bins having the scaling index $\alpha$ in the interval $[\alpha, \alpha+d \alpha]$ be $l^{-f(\alpha)} \rho(\alpha) d \alpha$, where $\rho(\alpha)$ is a smooth function. Then, the moment $C_{i}$ of the probability distribution can be written as :

$$
C_{i}(l)=\int_{\alpha_{\min }}^{\alpha_{\max }} l^{\alpha i-f(\alpha)} \rho(\alpha) d \alpha .
$$

Using the saddle-point approximation to the above integral one obtains for $1 \rightarrow 0$ :

$$
C_{i}(l) \sim l^{-\tau(i)}
$$

where $\tau(i) \equiv f\left(\alpha_{s p}\right)-i \alpha_{s p}$ and $\alpha_{s p}^{(i)}$ is the value where the function $i \alpha-f(\alpha)$ has the minimum.

The cut-off $\epsilon$ in the multifractal set means that we do not take into the calculation of the moments of the probability distribution those bins for which the density is smaller than the cut-off value. Recalling, that the scaling of density with the bin is governed by an index $\alpha$, one obtains a limiting value $\bar{\alpha}$ for this index :

$$
l^{\bar{\alpha}}=\epsilon .
$$

This means that for each length $l$ there exist a limiting value of the index $\bar{\alpha}$ so that bins with a larger value of the index $\alpha(\alpha>\bar{\alpha})$ do not contribute. Effectively, the 
limits of integration in (3.3.) are modified:

$$
r^{\prime}(l)=\int_{a m+n}^{n} l^{n i-f(\alpha)} \rho(\alpha) d \alpha
$$

This modifies the saddle-point estimate if $\alpha_{s p}>\vec{\alpha}$. $\Lambda$ s a result one obtains the following modification of the scaling law for the moments $C_{i}[103]$ :

$$
r^{\prime},(l) \sim \begin{cases}l^{-\tau(i)} & \text { if } \alpha_{s p}<\bar{\alpha}(l, c) . \\ l^{\alpha i-f(\alpha(l, c))} & \text { if } \alpha_{s p} \geq \alpha(l, c) .\end{cases}
$$

The above equation shows that the moments $C_{i}$ have a scaling behaviour down to some value of $l_{\text {lim }}$ and for smaller length they follow a pseudoscaling law given by the second case of the above equation. This limiting value of the bin length is given by :

$$
l_{\lim }=\epsilon^{1 / a_{s p}} .
$$

The scale $l_{l i m}$ where scaling breaks down depends on $\epsilon$ and, through $\alpha_{s p}$, also on the rank of the moment $i$. However, in the modified variables $\dot{C}_{\imath}, \Theta$ :

$$
\begin{aligned}
\tilde{C}_{i} & =\ln C_{i} / \ln \epsilon \\
\Theta & =\ln l / \ln \epsilon,
\end{aligned}
$$

all the dependencies of the type (3.36) for different values of the cut-off parameter are universal [103] :

$$
\dot{C}_{i}(\Theta) \sim \begin{cases}-\tau(i) \Theta & \text { if } \Theta<1 / \alpha_{s p} \\ i-\Theta f(1 / \Theta) & \text { if } \Theta \geq 1 / \alpha_{s p}\end{cases}
$$

Now we shall use the eqs. (3.36) and :

$$
F_{i}(M)=M^{i-1} F_{i}(1) \frac{C_{i}(M)}{\left(C_{1}(M)\right)^{i}} .
$$

to describe the behaviour of the SFMs of the modified $\alpha$-model. One gets straightforwardly :

$$
F_{i}(M) \sim \begin{cases}F_{i}(1) M^{i-1+\tau(i)} & \text { if } \alpha_{s p}^{(1)}<\bar{\alpha}(1 / M, \epsilon), \\ F_{i}(1) M^{i-1+r(i)+i \bar{\alpha}(1 / M, \epsilon)-i f(\bar{\alpha}(1 / M, c))} & \text { if } \alpha_{s p}^{(i)}<\bar{\alpha}(1 / M, \epsilon) \leq \alpha_{s p}^{(1)}, \\ F_{i}(1) M^{(i-1)(1-f(\hat{\alpha}(1 / M, \epsilon)))} & \text { if } \alpha_{s p}^{(i)} \geq \bar{\alpha}(1 / M, \epsilon) .\end{cases}
$$

From the above equation one can see that the pseudoscaling behaviour of the SFMs is mainly due to behaviour of the first moment $C_{1}$ which enters in eq. (3.36) for the SFMs. So that unlike for the multifractal moments $C_{t}$, where the moments of different rank have different $l_{\text {lim }}$, in the SFMs the pseudoscaling sets on at the scale given by $l_{l \mathrm{~m}}$ for $C_{1}$ :

$$
M_{\text {lim }}=\epsilon^{-1 / o_{s p}^{(1)}} \text {. }
$$

One can define similar variables as for the iwo-scale Cantor set studied in ref. [103]. The only difference is that before performing the multiscaling transformation (3.38) one has to rescale the moments and/or to rescale the length variable so that in the scaling 
regime $\left(l \geq h_{\text {lem }}\right)$ one has the scaling identity : $f,(l)=l^{-1}$. This can be achieved by studying the multiscaling in the variables: $F_{l}(I /) / F_{t}(1), . I=1 / l$. where the second variable is used by convention in the intermittency studies in order to have positive exponents. The multiscaling variables now take the form:

$$
\begin{array}{r}
G_{i}=\ln \left(F_{i}(M) / F_{i}(1)\right) / \ln (1 / \epsilon) \\
\Theta=\ln M / \ln (1 / \epsilon) .
\end{array}
$$

The SFMs for the random cascading model have a universal behaviour in these variables :

$$
G_{i}(\Theta) \sim \begin{cases}(i-1+\tau(i)) \Theta & \text { if } \Theta<1 / \alpha_{s p}^{(1)}, \\ (i-1+\tau(i)) \Theta+i-i \Theta f(1 / \Theta) & \text { if } 1 / \alpha_{s p}^{(1)} \leq \Theta<1 / \alpha_{s p}^{(i)} \\ (i-1) \Theta(1-f(1 / \Theta)) & \text { if } \Theta \geq 1 / \alpha_{s p}^{(i)} .\end{cases}
$$

Let us define the $\alpha$-model with the cut-off. In a given realization of the random cascade we stop the generation of the cascade in a given branch if the density associated with this branch is smaller than $\epsilon$. This means that the corresponding bin is assumed to have zero density and does not contribute to the moments of the density. Obviously, by dropping in each realization of the random cascade (event) these small density bins from the averages over all the realizations (events) we change the normalization of the density $\left(C_{1}(M) \neq 1\right)$. One has to take this change into account in order to have correctly normalized SFMs, i.e. in order to conserve the total density (energy). The rule of normalization implied by the eq. (3.40) corresponds to rescaling all the bins with density higher than $\epsilon$ by an amount corresponding to the density lost by dropping the bins with density smaller than the cut-off.

We have calculated numerically the SFMs for the $\alpha$-model $(\lambda=2)$ of random cascading with cut-off and with the probability distribution of the random factors $P(w)=0.5 \delta(w-1.2)+0.5 \delta(w-0.8)$. The results for different values of the cut-off parameter $\epsilon$ are shown in Fig. 1la. The higher the value of the cut-off the sooner the initial scaling behaviour $\left(F_{i}(M)=F_{i}(1) M^{\nu_{1}}\right)$ breaks down. According to the saddlepoint estimate (eq. 3.44) the dependence on the cut-off is universal and can be seen in the multiscaling variables $G_{i}$ and $\Theta$ (see Fig. $11 \mathrm{~b}$ ). As we can see the fluctuations caused by the infrared cut-off are dominant for small scales and the effective slopes are much higher than for the original (weak) intermittency. For the parameters in figs. $11 \mathrm{a}$ and $11 \mathrm{~b}$ the value of the intermittency index is $\nu_{2} \simeq 0.05$ whereas the slope in the pseudoscaling region taken from the last three full triangles $\left(\epsilon=3 \star 10^{-3}\right)$ is $\nu_{2}^{(p s e u d)} \simeq 3.4$. The numerical results show that the breaking of the scale-invariant regime in the SFMs occurs already for scales larger than the limiting scale $l_{\text {lim }}$. This is due to the deviations from the saddle-point approximation used in the derivation of eq. (3.36) [103]. However, the multiscaling dependence (3.44) is approximately universal for different cut-off parameters (Fig. 11b). Thus, the multiscaling analysis gives correct estimates of the cut-off, unlike the limiting scale in Fig. 11a.

In a high energy collision the value of the cut-off parameter should be given by the ratio of the mass of the particle to the total available energy. This leads to two predictions of the multiscaling for the high energy phenomenology : 
- the intermiteney shembl be stronger for hesaver hadrons

- the intermitlency should decrease with increasing c.m. energy of the collision.

The first prediction could be difficult to test because it requires the particle identification in the intermittency andysis and a sufficiently high multiplicities of heavy particles. Moreover, a spurious effect could be present due to the flavour-antiflavour correlations in phase-space. On the contrary, the second prediction can be easily tested. Actually, the results of the EMIV01 Collaboration on the $1 \mathrm{D}$ intermittency analysis in the nuclear collisions show that the intermittency indices decrease with the increasing incident energy $(14.6,60$ and $200 \mathrm{GeV})[68]$.

It would be interesting to test the multiscaling prediction in the $3 \mathrm{D}$ data for the SFMs at different energies. The multiscaling universality allows to find some universal dependence on the cut-off, i.e. on the energy and the hadronization scale. The fitted values of the cut-off parameters for several energies could give an estimate of the hadronization scale. The knowledge of this scale could serve to relate the partonparton correlation calculated in the perturbative QCD [105] to the correlations in the final state hadrons. The study of SFMs in the small phase-space cells provide a unique opportunity to observe the effect of the hadronization in the multiparticle final state.

In the case of the perturbative QCD cascade we also have an infrared cut-off which modifies behaviour of the correlations at small scales. Indeed, the dependence on the cut-off is also universal in the rescaled multiscaling variables (sect. 4.2.1). In the fragmentation inactivation binary cascading model, the cut-off appears naturally as due to the inactivation probability and the size of the monomers beyond which the fragmentation process must stop anyway (sect. 4.2.2). Also in this model the dependence on the cut-off is universal in the rescaled variables.

\subsubsection{Choice of the variable(s)}

Similarly as in the discussion of the problem of nonsingular parts in the correlations. the formal limit when the resolution goes to 0 does not depend on the choice of the variables for the singular part of the correlations. Finally one reaches the singularity which dominates and the intermittency indices are independent of the choice of the variables. However, at finite resolution the form of the correlations depends on the choice of the variables. Then the possible fits and results for the values of the intermittency indices depend on the variables. Several variables can be studied and naturally, one such set of variables is the rapidity, transverse momentum and the azimuthal angle. But even for this set of variables important changes are induced by the definition of the rapidity axis according to the beam axis or to the axis defined by the symmetry of the event. Other variables should be preferred if the expected correlations depend on the square of the momentum difference $\left(\vec{p}_{1}-\vec{p}_{2}\right)^{2}[89]$ or on the covariant four-momentum square difference $Q^{2}=-\left(p_{1}-p_{2}\right)^{2}[90]$. The covariant type of variables is important for the spatio-temporal intermittency [106] due to the causality constraint, which makes two space-time points which are not causally connected uncorrelated (sect. 4.1.1). Also sometimes the preferred variable is the invariant mass which is important e.g. for the correlated $e^{+} e^{-}$pairs. Finally, the QCD cascade has simple correlation functions in the relative angle variables $[5,107]$. 
Another possibility is the antsot ropy of the correlation functions, which may have different intermittency indices in different variables, and this both for factorizing and non-factorizing correlations. This type of correlations is expected in the Regge field theory and also in some scenarios of the fractal source in the Bose-Einstein correlations, if the space-time development is anisotropic. Certainly several components of the correlation functions have different important variables. The nicroscopic covariance induces the $Q^{2}$ variable, but many models have a preferred longitudinal direction corresponding to the rapidity and transverse variables. Generally, one should perform the analysis in several sets of variables, trying to identify the different sources of the correlations. An example of this is the elimination of the correlations coming from the $\epsilon^{+} e^{-}$conversion pairs [95].

\subsubsection{Event mixing in a simple model of fragmentation}

For many problems in astrophysics, nuclear physics or solid state physics, including the meteoritic distribution [108], the distribution of fragments produced in medium energy heavy-ions collisions, the polymer degradation [109], droplet breakup [110], crushing of rocks [111] etc., the knowledge of the cluster size distribution plays a fundamental role. Mekjian proposed a simple model [112], which provides an analytical framework for addressing many of those problems and, in particular, for understanding the relation between a power-law behaviour in the distribution of cluster-sizes found in many of those systems and the local fluctuations in the cluster-size distribution in single events.

In this statistical model one considers the partitions of $A$ particles into groups with $n_{j}$ clusters of $j$ elements $(j=1,2, \ldots, A)$. Various statistical models differ one from another by the choice of the weight of each partition $\left(n_{1}, n_{2}, \ldots, n_{A}\right)$, where $A \equiv \sum j n_{j}$. The weight function which leads to an exactly soluble model is [112] :

$$
W\left(\left\{n_{j}\right\}, x ; A\right)=\frac{A !}{n_{1} ! 1^{n_{1}} n_{2} ! 2^{n_{2}} \ldots n_{A} ! A^{n_{A}}} x^{m} \equiv M_{2}\left(\left\{n_{j}\right\} ; A\right) x^{m},
$$

where $m=\sum n_{j}$ is the fragment multiplicity and $x$ is a free parameter of the model. The probability of a given partition $\left(n_{1}, n_{2}, \ldots, n_{A}\right)$ is then :

$$
P\left(\left\{n_{j}\right\}, x ; A\right)=W\left(\left\{n_{j}\right\}, x ; A\right) / x(x+1) \ldots(x+A-1) .
$$

The statistical model given be the above weight function (3.45), with one value of the parameter $x$ for all $n_{j}^{\prime} s$, we shall call in the following the Mekjian model. This model is a special case of a more general statistical model characterized by the generalized weight function $[112]$ :

$$
W_{A}\left(\left\{n_{j}, x_{j}\right\}\right)=M_{2}\left(\left\{n_{j}\right\} ; A\right) \prod_{j=1}^{A} x_{j}^{n}
$$

Let us now define :

$$
W_{A}\left(\left\{x_{j}\right\}\right) \equiv \sum_{\pi_{A}} W_{A}\left(\left\{n_{j}, x_{j}\right\}\right)
$$


where $\pi_{A} \equiv\left(1^{n}, 2^{n}, \ldots \ldots 4^{\prime \prime}\right)$ specifies the decomposition of A elements. Then the generating function for $\mathrm{I}_{A}\left(\left\{x_{,}\right\}\right)$can be written as :

$$
\left.C_{r}\left(u,\left\{r_{j}\right\}\right)=\sum_{A=0}^{\lambda} H_{1}\left(\left\{r_{,}\right\}\right) \frac{u^{4}}{A !}=e^{\prime}\right)\left[u x_{1}+u^{2} \frac{x_{2}}{2}+u^{3} \frac{x_{3}}{3}+\ldots\right] .
$$

The distribution of clusters $\left\langle n_{1}\right\rangle$ is obtained by the ensemble averaging or, equivalently, by calculating the derivative of the generating function :

$$
<n_{i}>=\left.\frac{1}{x(x+1) \ldots(x+A-1)} \frac{\partial}{\partial x_{j}} \frac{\partial^{A}}{\partial u^{A}} G\left(u,\left\{x_{j^{\prime}}\right\}\right)\right|_{u=0 \text { and all } x_{,}=x}
$$

which gives:

$$
<n_{j}>=\frac{A !}{(A-j) !} \frac{x}{j} \frac{\Gamma(x+A-j)}{\Gamma(x+A)}
$$

Similarly one obtains $[113,114]$ :

$$
\begin{gathered}
<n_{j} n_{k}>= \begin{cases}\frac{A !}{(A-j-k) !} \frac{x^{2}}{j k} \frac{\Gamma(x+A-j-k)}{\Gamma(x+A)} & \text { for } j+k \leq A, \\
0 & \text { for } j+k>A \text { and } j \neq k .\end{cases} \\
<n_{j}\left(n_{j}-1\right)>= \begin{cases}\frac{A !}{(A-2 j) !} \frac{x^{2}}{j^{2}} \frac{\Gamma(x+A-2 j)}{\Gamma(x+A)} & \text { for } 2 j \leq A, \\
0 & \text { for } 2 j>A .\end{cases}
\end{gathered}
$$

or in the general case :

$$
\begin{aligned}
& <n_{j_{1}} \ldots n_{j_{k}}>= \begin{cases}\frac{A !}{\left(A-\sum j_{l}\right) !} \frac{x^{k}}{j_{1} \ldots j_{k}} \frac{\Gamma\left(x+A-\sum j_{l}\right)}{\Gamma(x+A)} & \text { for } \sum j_{l} \leq A, \\
0 & \text { for } \sum j_{l}>A \text { and } j_{n} \neq j_{m},\end{cases} \\
& <n_{j}\left(n_{j}-1\right) \ldots\left(n_{j}-p+1\right)>= \begin{cases}\frac{A !}{(A-p j) !} \frac{x^{p}}{j^{p}} \frac{\Gamma(x+A-p j)}{\Gamma(x+A)} & \text { for } p j \leq A, \\
0 & \text { for } p j>A .\end{cases}
\end{aligned}
$$

Formulas for the product of factorial moments of the number of fragments in different bins can be written analogously. When $x=1$, the frequency distribution of the fragmentation scheme as measured by its mass distribution, is given by a power-law $\left\langle n_{j}\right\rangle=j^{-\tau}$ with $\tau=1$ [112]. Moreover, the correlations take a very simple form :

$$
\begin{gathered}
<n_{j} n_{k}>=\left\{\begin{array}{ll}
<n_{j}><n_{k}> & \text { for } j+k \leq A \\
0 & \text { for } j+k>A \text { and } j \neq k
\end{array},\right. \\
<n_{j}\left(n_{j}-1\right)>= \begin{cases}<n_{j}>^{2} & \text { for } 2 j \leq A, \\
0 & \text { for } 2 j>A\end{cases} \\
<n_{j}\left(n_{j}-1\right)\left(n_{j}-2\right)>= \begin{cases}<n_{j}>^{3} & \text { for } 3 j \leq A, \\
0 & \text { for } 3 j>A\end{cases}
\end{gathered}
$$

and similarly for higher moments of the fragment-size distribution. Notice, that the correlations in the Mekjian model for $x=1 \quad(\tau=1)$ are nearly poissonian. The only difference between the above correlations and an uncorrelated, poissonian distribution of fragments, comes from the second case in eqs. (3.56) - (3.58), when the mass of the 
fragments exceeds the total mass of the system. Obviously, they vanish as $A \rightarrow \infty$. Sinnilar relations are valid also for a general average of the product of the numbers of fragments in different bins.

One can easily calculate analytically the normalized SFMs in this model [113]. In Fig. 12 we plot the normalized SFMs for a system of $A=216$ particles for different values of the tuning parameter. These are : $x=0.3$ corresponding approximately to the fused mode $(x<<1), x=0.5$ for the evaporation mode, the "critical" value $x=1$ for the scale-invariant power-law and $x=3$ which corresponds to the dissociation $(x>>1)$ [112]. We can see that the intermittent pattern of fluctuations does rot occur and in all regimes of the fragmentation the values of $\log F_{i}$ are negative and approach zero in small bins.

An important variation of SFMs in largest bins shows the influence of correlations due to the mass conservation, which diminish with increasing $A$ and the multiplicity distribution becomes closer to the poissonian one.

The presence of repulsive correlations due to the finiteness of the system leads to the question whether the SFM provide a good measure of fluctuations if the boundary effects and conservation laws are important. For any model of the fragmentation the following condition is true :

$$
<n_{j} n_{l}>=0 \text {, }
$$

for $j+l>A$. Whenever this condition influences many partitions, the uncorrelated distribution of the fragment sizes differs strongly from a Poisson distribution [1] . In this case the SFM remove more than only statistical fluctuations due to the random distribution of finite number of fragments in a given range of cluster sizes. The simplest way of taking into account that the mass of fragments in a fragmentation event is less or equal to $A$ is to redefine the normalization factor :

$$
\begin{aligned}
\bar{R}_{i}= & M^{-1} \sum_{j=1}^{M} \frac{1}{\delta s_{j}{ }^{j}} \int_{\delta s_{,}} d s_{1} \ldots \int_{\delta s_{,}} d s_{i} \\
& \left(\rho\left(s_{1}\right) \ldots \rho\left(s_{i}\right) \Theta\left(A-s_{1}-\ldots-s_{i}\right)\right) /\left(\Delta s^{-1} \int_{\Delta s} \rho(s) d s\right)^{i} .
\end{aligned}
$$

The above expression is just the SFM for the Mekjian model, because of the relation :

$$
\rho_{i}\left(s_{1}, \ldots, s_{i}\right)=\rho\left(s_{1}\right) \ldots \rho\left(s_{i}\right) \Theta\left(A-s_{1}-\ldots-s_{i}\right) .
$$

The normalized factorial moments for the Mekjian model are : $F_{i}=\bar{R}_{i} / R_{i}$ and, obviously, using the definition (3.60) for the normalization factor would give $F_{i}=1$ for all bins. However, as we have shown the difference between $\bar{R}_{i}$ and $R_{i}$ is very small in the case of the Mekjian model for which at the scaling point $\rho(s) \sim s^{-1}$ and it is even smaller for the fragment size distribution in the percolation for which $\rho(s) \sim s^{-\tau}$ and $\tau \geq 2$. However this correction could be important if the one particle distribution is approximately constant $\rho(s) \simeq \operatorname{const}(s)$.

In the Mekjian model, one investigates the fluctuation pattern for events of a given class, defined by the specific value of the tuning parameter $x$. In the experimental situation, such a clean separation of events in different classes is difficult and usually depends on certain supplementary assumptions about the dynamics of the process. 
Hence. the analyzed sample mal contain events associated with the varions "violence" of the collision. Mixing in one ensemble events of different physical nature. characterized by different control parameters and hence by different inclusive fragment distribution $N_{s}(x)$. is equivalent to introducing new, in general attractive, correlations and the distribution of fragments in this case may differ from the Poisson distribution strongly. In the language of the Mekjian model, where the partition probabilities are controlled by a single parameter $x$, this would correspond to mixing different values of $r$. Since the probability of a given partition $\left(n_{1}, n_{2}, \ldots, n_{A}\right)$ is a linear combination of $P\left(\left\{n_{j}\right\}, x ; A\right)$ for different $x$ :

$$
P\left(\left\{n_{j}\right\} ; A\right)=\sum_{x} \alpha(x) P\left(\left\{n_{j}\right\}, x ; A\right) \quad,
$$

with the weight function $\alpha(x)$ :

$$
\begin{aligned}
& \sum_{x} \alpha(x)=1 \\
& <n_{j}>\equiv \sum_{x} \alpha(x)<n_{J}>_{r},
\end{aligned}
$$

the factorial moment : $F_{k}(\delta s ; j) \equiv<n_{j}\left(n_{j}-1\right) \ldots\left(n_{j}-k+1\right)>$ in any bin $j$ of size $\delta s$. is a linear combination of factorial moments $\left.F_{k: x}(\delta s ; j)<n_{j}\left(n_{j}-1\right) \ldots(n j-k+1)\right\rangle_{x}$ for each $x$ separately. Therefore, the SFMs corrected for the shape of the inclusive distribution :

$$
\begin{aligned}
\dot{F}_{k} & =\frac{\sum_{j=1}^{A / \delta s}\left(\sum_{x} \alpha(x) F_{k, x}(\delta s ; j)\right)}{\sum_{j=1}^{A / \delta s}\left(\sum_{x} \alpha(x)<n_{j}>_{x}\right)^{k}} \\
& =\sum_{j=1}^{A / \delta s} \sum_{x}\left(\frac{\alpha(x)<n_{j}>_{x}{ }^{k}}{\sum_{j=1}^{A / \delta s}<n_{j}>^{k}}\right)\left(\frac{F_{k, x}(\delta s ; j)}{<n_{j}>_{x}{ }^{k}}\right)
\end{aligned}
$$

exhibit a nonlinear dependence on the weight function $\alpha(x)[113,114,115]$. Consequently, even if $F_{k, r}(\delta s, j) /\left(<n_{j}>_{x}\right)^{k} \sim 1$ for any bin $j$ of size $\delta s$ and for each $x$, the resulting SFM $\tilde{F}_{k}$ can be large if $\alpha(x)$ is small for $x$ corresponding to the largest contribution of $\alpha(x)<n_{j}>_{x}[113,114]$. This mixing leads mainly to an overall shift in the value of SFM in any bin and, therefore, it is useful to renormalize the values of SFMs in all bins by their value in the largest bin.

Fig. 13 presents the results of calculations for the statistical ensemble composed of events in the range of tuning parameters from $x_{\min }=0.5$ to $x_{\max }=3.5$ and weighted with the same constant factor $\alpha(x)$ for all $x . F_{i}(i=2,3)$ in Fig. 13 exhibits a similar non-intermittent dependence on the bin size as seen before in Fig. 12 but now the values of the moments are positive in the whole range of bin-sizes and the dependence of the SFMs on the scale is stronger. An example was given of event mixing of different classes of fragmentation events which gives intermittent behaviour of the SFMs, even though each of the different classes of events separately do not have such correlations [116].

One should underline that mixing of events associated e.g. with different fragment multiplicities or excitation energies result also from a physical process such as the 
percolation phase transition or the shattering phase transition in random cascading (see the discussion in chapter 1). In these cases, restricting the ensemble of events to those corresponding to a fixed multiplicity or excitation energy would mean neglecting physical correlations characterizing the studied process. In these cases, for a fixed value of a control parameter such as e.g. the bond activation probability in percolation or the exponents of the fragmentation and inactivation kernels in the random cascading, one obtains a distribution of fragment multiplicities (excitation energies) which are characteristic for the studied phenomenon and associated to it the critical phenomenon. We shall return to this discussion in the next chapter while discussing the fluctuation features of both the percolation process and the fragmentation-inactivation binary cascading process. 


\section{Models}

Although the amount of experimental results concerning the correlations at very small scales is already quite large, the theoretical understanding of the small scales phenomena is still lacking. The experiments recently confirmed that the Bose-Einstein correlations are dominant in the intermittency effect in 3D correlations. The origin of such power-law structures in the emitting sources is still to be understood. The phase-transition explanation of the strong fluctuations is still discussed and recently it got new motivations from the chiral phase transition models [117]. However up to now there is no data in ultrarelativistic leavy-ion collisions that would support this hypothesis. The cascade structure of the perturbative QCD was also analyzed and some scale-invariant regimes were found in the correlation functions [5]. At lower excitation energies, where the copious production of intermediate-mass fragments is a dominant deexcitation mechanism, several scenarios are considered. Among "static" ones, there are the equilibrium statistical multifragmentation [39], the percolation [40] and the equilibrium binary fragmentation models like the GEMINI. Another scenarios, which stress the non-equilibrium aspects of the fragment production are the volume (spinodal) [118] and surface [119] instabilities as well as the non-equilibrium cascading [120] . In this latter scenario, one expects that non-equilibrium dissipative processes in the early stage of collision yield the instable, fragmenting hot residuum in a self-organized state and the process exhibits critical aspect of a shattering transition.

\subsection{Phase transition}

\subsubsection{High energy phase transition}

It was claimed that the presence of non statistical fluctuations in the spectra of hadrons produced in ultrarelativistic nuclear collisions could be a signal of the quarkgluon plasma formation $[21,1]$. The effect of non-statistical fluctuations can be extracted and the comparison between different processes is possible using the method of the SFMs $[1,2]$. The experimental data in $e^{+} e^{-}$and hadron-hadron collisions confirm the existence of such fluctuations and the dependence of SFM on the resolution in rapidity can be fitted using a power-law relation. In the case of a large number $N$ of independent fluctuating sources, the superimposed distributions would follow a similar law with the slopes $\nu_{i}$ which are $N$ times smaller than for one source $[75,121,122]$. This motivates the so called Bialas plot, i.e. the intermittency slopes versus the particle rapidity density $\frac{d n}{d y}$. Comparing on such a plot different nuclear collisions at similar energies leads to the conclusion that the intermittency slopes are anomalously high for heavy targets [68]. The discussion of the independent collision model was performed by (apella et al. [121] who concluded that this model gives values of the intermittency slopes 4-20 times too low and, consequently, the observed intermittency slopes in nuclear collisions cannot be interpreted as an effect of superimposed nucleon-nucleon collisions. One should notice that even if the mechanism responsible for the fluctuations 
in nucleus-mucleus collisions is different than in hadron-hadron collisions, still there is a lack of consistency in the observed parameters for different projectiles (P, O, S) [90]. In Table 1. we show the experimental data for $F_{2}$ and $F_{3}$ for three projectiles [67] as well as the predictions for the projectile nucleus $A$ from the p-emulsion data which are rescaled in accordance with the change of the mean multiplicity :

$$
F_{i}^{A}=\frac{F_{i}^{p}<N_{p}>}{<N_{A}>}
$$

As it was discussed [90], the observed decrease of the intermittency slopes is not as large as the corresponding increase of the multiplicity. One of the mechanisms which could explain such relations between the SFMs in different systems are the Bose-Einstein correlations [123]. These correlations are indeed collective and from a naive dimensional argument one expects that the local "slope" of the Bose-Einstein correlation function in momentum is inversely proportional to the linear size of the system and the mean multiplicity grows certainly like a higher power of the linear size of the system. This could be an explanation of the 3D data which is of the Bose-Einstein type. There could be another explanation of this effect in the 1D data if the system undergoes a phase-transition. This would lead to collective correlations in rapidity and not in the $3 \mathrm{D}$ momentum space if the intermittency present, in the space-time could be observed only in rapidity according to the Bjorken's scenario. Bialas and Hwa proposed that the intermittency patterns of fluctuations in the nuclear collisions could be a consequence of a higher order phase-transition from quark-gluon plasma into hadrons [83]. More generally, it could also be understood as an effect of some strong space-time correlations in the nuclear dynamics. In the picture of partons and strings it means that the main contribution to the observed intermittency patterns does not come from the stage of parton cascade or independent string fragmentation but instead from the stage of dynamical evolution of the interacting string network with a possible phase-transition similar to the percolation [124].

In ref. [90], the two-particle density in space-time was assumed to be a scaleinvariant function, like in the critical point of a second order phase-transition. The correlation function at the proper time $\tau_{2}$ of the freeze out, can be written in the covariant form :

$$
d_{2}\left(\overrightarrow{r_{1}}, \overrightarrow{r_{2}}\right)= \begin{cases}\left.C_{2}\left(\tau_{12}\right)^{-\nu_{2}}\right|_{t_{1}=\sqrt{\tau_{2}^{2}+\vec{r}_{i}^{2}}} & \text { for } \tau_{12}<\tau_{c o r r} \\ C_{2}\left(\tau_{\text {corr }}\right)^{-\nu_{2}} & \text { for } \tau_{12} \geq \tau_{c o r r}\end{cases}
$$

where $\tau_{12} \equiv \sqrt{\left(\vec{r}_{2}-\vec{r}_{1}\right)^{2}-\left(t_{2}-t_{1}\right)^{2}}$ and $\tau_{\text {corr }}$ is the range of the correlations. The finite range of the correlations in this model is exclusively due to the causality constraints. Assuming that the phase-transition takes place at a certain proper time $\tau_{1}$ and that the correlations cease to build at some other value of the proper time $\tau_{2}$, one obtains $\tau_{\text {corr }}=\left(\tau_{2}^{2}-\tau_{1}^{2}\right) / \tau_{1}$ for the range of the fluctuations ( see Fig. 14). The intermittency exponent $\nu_{2}$ describes the strength of the singular correlations and is given by the nature of the mechanism by which the spatio-temporal intermittency is build up. In our calculation we took $\nu_{2}=1.0$.

For the dynamics of the reaction we assume the Bjorken's scaling in the longitudinal direction. The ideal inside-outside correspondence will be smeared out, to simulate 
realistically the dyamies of the interactions at 200 (iel/mel. The other non-ideal effect taken inte acrount in the presence of the long lived resonances. Here we assume that a given part $(1-q)$ of the observed pions come from the decay of such resonances 1 . The decay is assumed to be isotropic in the rest frame of the resonance, in which case the rapidity distribution is $1 / \cosh ^{2} y$. Such a distribution is well approximated by a gaussian distribution with the half-width equal 0.75 . The one-particle density is assumed to be constant inside a tube of radius $R_{t u}$ and zero outside. The radius of the tube is given by the geometry of the reaction, i.e. by the impact parameter and the projectile radius. The interaction region for central collisions is determined by the radius of the smaller (projectile) nucleus, $R_{t u}=r_{0} A^{1 / 3}$. Integrating the twoparticle reduced density over transverse radii and azimuthal angles, one obtains the one-dimensional reduced correlations :

$$
\begin{gathered}
c_{2}^{(1-d i m)}\left(y_{1}, y_{2}\right)=\left(\pi^{-2} R_{t u}^{-4}\right) \int_{0}^{R_{t u}} R_{1} d R_{1} \int_{0}^{R_{t u}} R_{2} d R_{2} \int_{-\pi}^{\pi} d \phi_{1} \int_{-\pi}^{\pi} d \phi_{2} \\
c_{2}\left(R_{1}, \phi_{1}, y_{1} ; R_{2}, \phi_{2}, y_{2}\right) .
\end{gathered}
$$

This represents the two-particle reduced correlations of the space-time rapidity distribution of the sources. Following our assumptions, this density (4.3) corresponds only approximately to the kinematical rapidity distribution. In order to obtain the distribution in the kinematical rapidity one should fold the space-time rapidity distribution with the phase-space distribution, which we take in the following form [125]:

$$
f\left(y, y^{(s t)}\right)=\frac{\exp \left(-\left(y-y^{(s t)}\right)^{2} / 2 \sigma^{2}\right)}{\sigma \sqrt{2 \pi}} .
$$

In the case of the ideal inside-outside cascade, the phase-space distribution is given by the $\delta$ function :

$$
f\left(y, y^{(s t)}\right)=\delta\left(y-y^{(s t)}\right) .
$$

The parameter $\sigma$ in the above equation represents the width of the smearing of the rapidity distribution. The correlation function in rapidity is then :

$$
c_{2}^{(k i n)}\left(y_{1}, y_{2}\right)=\int_{-\Delta Y_{s t} / 2}^{\Delta Y_{s t} / 2} d y_{1}^{(s t)} \int_{-\Delta Y_{s t} / 2}^{\Delta Y_{s t} / 2} d y_{2}^{(s t)} c_{2}^{(1-d i m)}\left(y_{1}^{(s t)}, y_{2}^{(s t)}\right) f\left(y_{1}, y_{1}^{(s t)}\right) f\left(y_{2}, y_{2}^{(s t)}\right)
$$

This is the kinematical rapidity distribution of the sources of the final pions. As we already mentioned, only about $q=0.25-0.5$ of the total number of pions are produced directly and the rest comes from the resonances decays. Taking this into account, the two-particle rapidity distribution for pions can be written as follows :

$$
\begin{aligned}
& c_{2}^{(\pi)}\left(y_{1}, y_{2}\right)=\int_{-\Delta Y / 2}^{\Delta Y / 2} d y_{1}^{\prime} \int_{-\Delta Y / 2}^{\Delta Y / 2} d y_{2}^{\prime} d_{2}^{(k i n)}\left(y_{1}^{\prime}, y_{2}^{\prime}\right) \\
& \left(q \delta\left(y_{1}-y_{1}^{\prime}\right)+(1-q) \rho^{(i s o)}\left(y_{1}-y_{1}^{\prime}\right)\right)\left(q \delta\left(y_{2}-y_{2}^{\prime}\right)+(1-q) \rho^{(i s o)}\left(y_{2}-y_{2}^{\prime}\right)\right) .
\end{aligned}
$$

The decay distribution of a resonance $\rho^{(i s o)}$ in the above equation is approximated by :

$$
\rho^{(\imath s o)}(y)=\frac{\operatorname{cxp}\left(-y^{2} / 1.125\right)}{0.75 \sqrt{2 \pi}} .
$$

\footnotetext{
${ }^{1}$ Some detailed results from the ${ }^{+{ }_{t}-}$ annihilation indicate that the effect of the resonance decay effectively does not change (or even increase) the fluctuations [55], so it is not excluded that the value $q=1$ could be better suited for this calculation ( see Fig. 18 ).
} 
From the two-paticle resheed density one obtains direetly the second SFM :

$$
r_{2}=\frac{1}{(\delta y)^{2}} \int_{0}^{0 y} d y_{1} \int_{0}^{\delta y} d y_{2} c_{2}^{(\pi)}\left(y_{1} \cdot y_{2}\right)
$$

The results for $F_{2}(\delta y)$ in the range $\delta y=0.1-1$ are plotted in Fig. 15. As one can see the results depend strongly on the assumed freeze-out time $\tau_{2}$. The resulting slopes $\nu_{2}$ of the SFM are of the same order of magnitude as observed experimentally. However, the detailed comparison is not meaningful because the calculated slopes $\nu_{2}$ depend sensitively not only on the projectile and the impact parameter but also on the unobservable parameters such as the correlation range $\tau_{c o r r}$, percentage of the direct pions $q$ and the width $\sigma$ of the rapidity spread around the Bjorken's solution. So the dependence of the SFMs on the projectile and the impact parameter as calculated in this model, can be treated only as a qualitative relation. In Table 1, one can see the comparison of the experimental data with results of calculations obtained for the freeze-out time $\tau_{2}=6 \mathrm{fm}$. Even though the numerical values of the intermittency slopes $\nu_{2}$ are systematically underestimated, they depend on the projectile less strongly than the inverse of the mean multiplicity (eq. 4.1).

For comparison, we are plotting in Fig. 16 the similar results for a different set of parameters. The proportion of the direct pions is now set to $q=0.5$ and the half-width of the smearing of the rapidity distribution is $\sigma=0.5$. The resulting SFMs have a similar dependence on the rapidity bin width $\delta y$ as those plotted in Fig. 15. This shows the difficulty in making a quantitative comparison with the data, without having a precise estimate of the unknown parameters.

In Table 1. we make a comparison between central nucleus-nucleus collisions in the model (the constant radius of the interaction region) and a sample of central and medium parameter collisions in the experiment. This could be another effect explaining the stronger attenuation of the calculated slopes. On the other side, all ultrarelativistic proton-nucleus collisions are in our model central. So, the model gives relations for central collisions of different projectiles and the experiment gives relations between central proton-nucleus and a mixture of central and medium impact parameter nucleusnucleus collisions.

Following those arguments, one can take the relations between the "slopes" of the SFMs in our model (see the Table 1.) as the lower bound. Nevertheless, these relations are in much better agreement to the relations followed by the experimental data on $F_{2}$ than the independent superposition models, and serve as a schematic explanation of the observed relations between intermittency exponents for different projectiles and targets. It would be interesting to compare the results of the model with the experimental results for a sample of only central collisions for different projectiles.

This tendency of the results of the model of spatio-temporal intermittency could certainly be made closer to the experimental data, if we would allow for a projectile dependence on both the spatial and the temporal extensions of the interaction region. This means that taking larger freeze-out time $\tau_{2}$ for heavier projectiles, one can obtain even weaker attenuation of the slopes with the mass of the interacting nucleus. The recent results of the NA35 Collaboration [126] on the time of the pion emission in ultrarelativistic collisions gives a very high value of the freeze-out time of the order of $20 \mathrm{fm} / \mathrm{c}$. The lifetime of the pion source in the mid-rapidity was found to be much 
longer in the asymmetric collisions $(20 \mathrm{fm} / \mathrm{c}$ for ()$-\mathrm{A}$ ) than in the symmetrie collisions $(6 \mathrm{fm} / \mathrm{r}$ for $S-S)[126]$. This strong increase of the emission time with the asymmetry of the collision can explain in the framework of the present model the increase of the slopes of the SFMk with the increase of the mass of the target. as seen by the EMt 01 Collaboration [68]. As said before, the interaction time in the asymmetric collisions can take a value of $20 \mathrm{fm} / \mathrm{c}$ and, as one can see in figs. 17 and 18 , it gives a substantial increase of the intermittency signal.

The hypothesis that the system during the collision undergoes a second order phase transition, allows the introduction of simple, phenomenological (inzburg - Landau Hamiltonians for the description of the system at and around the critical point [127]. These approaches allow to relate the quantities calculated using these Hamiltonians. e.g. the critical indices and the correlation length, to the analogous observables in the distribution of the particles produced in ultrarelativistic nuclear collisions. However, if the critical correlations are present in space-time, one needs the assumption of the Bjorken's scaling in order to observe some of those correlations also in the moment a of the produced particles. Then, all non-ideal effects discussed above could reduce significantly or even wash out the critical scale-invariant correlations. A more convincing signal would be the simultaneous observation of the fluctuations in rapidity which due to the non-ideal effects would be no longer scale-invariant, and the obscrvation of power-law tails in the Bose-Einstein correlations indicating the presence of singular space-time correlations in the emitting sources. Of course, the precise form of the Hamiltonian chosen to describe the critical properties of the system remains an open question. In the Ginzburg - Landau theory it is a simple scalar Hamiltonian as in ref. [127], whereas for the description of the chiral condensate as in ref. [117], it is an $O(4)$ Hamiltonian.

Another interesting problem is the behaviour of the fluctuations within the relativistic hydrodynamical models. This question was addressed in different context in ref. [128], where the diffusion of plasma droplets through the expansion was discussed. We studied the fluctuations in the $(1+1)$-D hydrodynamical model of nucleus-nucleus collisions with hadronization sources described in ref. [129]. The model was modified by the introduction of the randomness of a gaussian or fractal type in the sources. The hydrodynamical evolution of the system was studied by numerical integration of the equations of the relativistic hydrodynamics in two dimensions :

$$
\partial_{\mu} T^{\mu \nu}=\Sigma^{\nu}
$$

where $T^{\mu \nu}$ is the energy-momentum tensor:

$$
T^{\mu \nu}=-p g^{\mu \nu}+(\epsilon+p) u^{\mu \nu} u^{\nu}
$$

with $u^{\mu}$, $\epsilon$ and $p$ being the fluid local velocity, energy density and pressure. In the studied example we assume a simple equation of state which yields the relation :

$$
\partial p=c_{s}^{2} \partial \epsilon,
$$

with a constant sound velocity $c_{s}$. Rewriting these equations in the variables:

$$
\hat{\imath}=\frac{1}{2} \log \left(x^{+} x^{-} / \tau_{0}^{2}\right)
$$


and

$$
y=\frac{1}{2} \log \left(x^{+} / x^{-}\right)
$$

one obtains $[129]$ :

$$
\begin{aligned}
\left(\partial_{i}+\bar{v} \partial_{y}\right) \epsilon+(\epsilon+p)\left(\nu \partial_{i}+\partial_{y}\right) \Theta & =\frac{\exp (\hat{t})}{\cosh (\Theta-y)}\left(\Sigma^{0} \cosh \Theta-\Sigma^{1} \sinh \Theta\right) \\
\left(\bar{v} \partial_{\hat{t}}+\partial_{y}\right) p+(\epsilon+p)\left(\partial_{i}+\imath \partial_{y}\right) \Theta & =\frac{\exp (\hat{t})}{\cosh (\Theta-y)}\left(-\Sigma^{0} \sinh \Theta+\Sigma^{1} \cosh \Theta\right),
\end{aligned}
$$

where

$$
\Theta=\frac{1}{2} \log \left(\frac{1+v}{1-v}\right)
$$

and

$$
\bar{v}=\tanh (\Theta-y) .
$$

The sources $\Sigma^{\nu}$ represent the hadrons forming after the first nucleon-nucleon collisions, so that they are a kind of the retarded map of the particle production in direct collisions. For the details see ref. [129], where a functional form of the sources is proposed. All the parameters and initial conditions are taken from ref. [129] and correspond to central $\mathrm{U}-\mathrm{U}$ collision with $400 \mathrm{GeV} / \mathrm{A}$ energy. Those sources are then modified by applying a random factor $\zeta(y)$ :

$$
\Sigma_{\nu}^{(r)}(\hat{t}, y)=\Sigma_{\nu}(\hat{t}, y) \zeta(y)
$$

and we take :

$$
\zeta(y)=\exp \left(\xi(y) s-1 / 2 s^{2}\right)
$$

with a normally distributed random variable $\xi(y)$, or a self-similar distribution in ra pidity $\zeta(y)$. The simulation shows that the fluctuations induced by the random sources giving the initial conditions for the hydrodynamical evolution are strongly damped in the evolution. It reflects the stability of the Bjorken's solution of the two-dimensional hydrodynamical equations. The energy density is shown in figs. $19 \mathrm{a}$ and $19 \mathrm{~b}$ for three evolution times.

The analysis of the moments of the energy distribution shows a systematic flattening of the distributions (Figs. 20a and 20b). The fitted slopes of SFMs decrease rapidly during the hydrodynamical evolution, i.e. the energy distribution smooths out and its dimension approaches 1 .

This mechanism can cause another weakening of the intermittency patterns. The possible appearance of fractal or fluctuating distributions due to a random hadronization would be strongly damped during the hydrodynamical expansion. The assumed independence of the sources on time is of course the most optimistic hypothesis. If we superpose independent random sources at different times in the same rapidity interval, the effect will then be only weaker due to averaging.

The detailed study of the damping of the fluctuations can be performed, for a simpler situation of the hydrodynamical evolution of the initially fractal distribution. For the initial distribution we take a self-similar energy density distribution in a range of rapidity scales, following the $p$-model distribution [75]. For the rapidity distribution $\Theta(y)$ we take the scaling initial condition $\Theta(y, t=0)=y$. One can observe that the 
intermitency shomen are strongly reduced atready after the first fm/e of the evolution. As was already mentioned. this damping of the fluctuations is a result of the stability of the $(1+1)-1)$ scaling solution of the perfect fluid hydrodynamics [130]. The general problem of the stability of the scaling solution in $(1+n)$-I) for the relativistic viscous fluid was addressed in rof. [131] and the limits on the viscosity coefficients were found for which the scaling solution is stable. There exist however some regions of the parameters were the scaling motion becomes unstable. The authors of ref. [131], using the results of Hosoya and Kajantie [132] for the viscosity coefficients of the quark-ghon plasma, found that the stability condition given by the Reynold's number $R>1$ is well satisfied. For example, for the initial temperature of the plasma $T=300 \mathrm{MeV}$, one finds $R=7.22$ for $\alpha_{s}=0.3$ and $R=1.34$ for $\alpha_{s}=0.1$. However, the calculation of Baym et al. [133] predicts a larger value of the plasma viscosity and the corresponding Reynold's number is $R=1.79$ for $\alpha_{s}=0.3$ and $R=0.33$ for $\alpha_{s}=0.1$. This value can be even lower for a lower value of the initial temperature. According to this prediction [133], the unstable region $R<1$ could be approached in the nucleus-nucleus collisions and could produce many unexpected results such as the increase of the fluctuations in the rapidity distributions. We have not checked numerically the damping of the fluctuations in the viscous fluid. Taking a lower value of the sound velocity than for the ideal equation of state $\left(c_{s}=1 / \sqrt{3} c\right)$, we have found a slower damping of the fluctuations during the hydrodynamical evolution.

The turbulent origin of the fluctuations was proposed to explain the fluctuations in the distributions of galaxies in the universe [134]. However, in the case of the heavyion collision, the short time of the hydrodynamical evolution provides some constraint on the space extension of those fluctuations. Taking for the time of the evolution $t=4 \mathrm{fm} / \mathrm{c}$ and for the sound velocity $c_{s}=1 / \sqrt{3} c$, one finds the upper limit on the extension of an eddy $r_{\text {eddy }}=0.7 \mathrm{fm}$ (one full revolution of the eddy with the sound velocity). Larger eddies simply have no time to build up. So even if the hydrodynamical evolution of a collision would give strong fluctuations, these cannot be thought of as the fully developed turbulence in a broad range of scales.

In conclusion, the ideal hydrodynamics is damping the fluctuations very rapidly. We can deduce that if the observed multiplicity fluctuations come from the stage before or during the hydrodynamical evolution, then this evolution must be very short or the viscosity coefficients must be in the range of values where the scaling solution of relativistic hydrodynamics is unstable [131]. This means that the fluctuations are produced during the final stage of the collision, just before the freeze out, or that the hydrodynamics describing the reaction is near or in the unstable region. In this latter case, hydrodynamics could be even the dominant source of fluctuations.

\subsubsection{Percolation model in 1D}

The fragment size distributions following nuclear multifragmentation at low and intermediate bombarding energies, exhibit features similar to those seen in percolation models $[37,40,135]$ in which, close to the critical regime, the fragment (cluster) size distribution has the form:

$$
N(s, \varepsilon) \sim s^{-\top} f\left(\varepsilon s^{\sigma}\right)
$$


where $f\left(E s^{\sigma}\right)$ is the scaling function, and $z$ is a variable characterizing "distance" from the critical point. E can be the difference to the critical temperature $T$ " in a thermal phase transition or the "distance" to critical fraction of active bonds $q_{\text {c or occupied }}$ sites $p_{c}$ in percolation models. $s$ in the above formula is the size of the finite cluster and $\tau, \sigma$ are critical exponents. For $\varepsilon<0$ one infinite cluster exists, whereas for $\varepsilon>0$ one finds only droplets. The extent to which nuclear systems in breakup processes exhibit the same kind of critical behaviour as the liquid - gas transition or percolation transition on the lattice is largely an open problem. Investigation of the intermittency in both the nuclear multifragmentation and the percolation model might be helpful in settling this problem. Below, we shall discuss the features of this model in $1 \mathrm{D}$ and $3 \mathrm{D}$ with the aim to understand the salient features of the fluctuations in the fragment-size distribution at around the critical point.

Grouping of particles into composites of different sizes in the Mekjian canonical ensemble model is described by the choice of the weight function[112]. In this sense this model contains "non-interacting" constituents. On the contrary, in the Ising or percolation models, one specifies the probabilistic local connectivity rule ("the interaction") between particles from which the complicated and unknown in general weight function of each partition can be constructed. As for many problems of physics, the percolation problem can be solved exactly only in the one and in the infinite (Bethe lattice ) dimensions.

Let us consider the bond percolation on a $1 \mathrm{D}$ chain of sites of length $A$ with periodic boundary conditions. Neighbouring sites are connected by bonds which are randomly activated with the probability $q$. A fragment with size $1<s \leq A$ is defined as an ensemble of neighbouring sites connected by active bonds. The percolation threshold in $1 \mathrm{D}$ is $q_{c}=1$, i.e. in this model it is only the point $q_{c}=1$ for which the size distribution is singular. In this sense $1 \mathrm{D}$ percolation model is not critical. The $1 \mathrm{D}$ clusters also do not posses any nontrivial scaling properties as is the case for the infinite cluster in 3D percolation. Nevertheless, the fragment formation in the $1 \mathrm{D}$ model has some similarities to the percolation in nontrivial dimensions. Due to its simplicity, the SFMs can be calculated exactly and the origin of the fragment-size correlations can be better understood.

For a given bond activation probability $q$, the mean number of the clusters of size $j$ is :

$$
<n_{j}>=\left\{\begin{array}{ll}
A(1-q)^{2} q^{j-1} & \text { for } j<A \\
A(1-q) q^{A-1}+q^{A} & \text { for } j=A
\end{array} .\right.
$$

Similarly, the average of the product of the number of fragments of two sizes can be calculated :

$$
<n_{3} n_{k}>=\left\{\begin{array}{lr}
A(1-q)^{2} q^{j+k-2} & \\
\left(2(1-q)+(A-j-k)(1-q)^{2}\right) & \text { for } j+k<A, \\
A q^{j+k-2}(1-q)^{2} & \text { for } j+k=A, \\
0 & \text { for } j+k>A
\end{array},\right.
$$




$$
<n_{,}\left(n_{,}-1\right)>=\left\{\begin{array}{ll}
1(1-q)^{2} q^{2-2} & \\
\left(2(1-q)+(A-2 j)(1-q)^{2}\right) & \text { for } 2 j<1 . \\
A q^{2 j-2}(1-q)^{2} & \text { for } 2 j=A . \\
0 & \text { for } 2 j>A .
\end{array} .\right.
$$

The evaluation of higher products proceeds analogously[113]. Having calculated the averages $\left\langle n_{J_{1}} n_{J_{2}} \ldots\right\rangle$ of the number of fragments of different sizes one can proceed to calculate the SFM. The results for $F_{2}$ and $F_{3}$ are shown in Fig. 21 for the system of $A=216$ particles. Three plots in Fig. 21 correspond to the three different bond activation probabilities $q=0.5,0.8,0.9$. The SFMs show an increase when going to small bins in the sizes of fragments, but this increase is not of a self-similar type. The dependence of the SFM on the bin size $\delta s$ ! las a jump at some bin width $\delta_{j u m p} s$ and saturates both for small and large intervals. The source of this behaviour can be found by comparing the correlations given by eqs. (4.21) and (4.22) with those of the poissonian type for the uncorrelated system. The two fragment mass correlation is :

$$
c_{j k} \equiv \frac{\left\langle n_{j} n_{k}>-<n_{j}><n_{k}>\right.}{<n_{j}><n_{k}>}=\frac{1}{A} \frac{2}{(1-q)-(j+k+1)},
$$

for $j+k<A$. The cumulant $c_{j k}$ is built up of two terms. The first term corresponds to the configuration where the two fragments under consideration are separated only by one empty link and then the probability of such configuration has a factor $(1-q)^{3}$ to account for the empty links and not $(1-q)^{4}$ as in the product $\left\langle n_{j}\right\rangle\left\langle n_{k}\right\rangle$. This is the only configuration where the correlations are attractive. This term becomes more important when $q$ approaches 1 . The second term accounts for the effect of the finite size of the system where the two fragments are chosen and gives rise to repulsive correlations. There is also another source of repulsive correlations, namely the case when $j+k>A$. In this case $c_{j k}=-1$. This effect was found already before ( see sect. 3.4.6) for the Mekjian model and it was shown to be small. This is also true for any model where the fragment-size distribution decreases rapidly, as is the case of the percolation in one or more dimensions. Thus, in the following, we will restrict the considerations to the case $j+k<A$. Using eq. (4.23) one may derive the relation for the scales where the attractive correlations dominate :

$$
\delta s \equiv k-j<\frac{2}{1-q}-2 j-1(k \geq j) .
$$

Taking $j=1$ in (4.24) one obtains an estimate of the scale where the attractive interactions show up :

$$
\ln \delta_{j u m p} s=\ln \left(\frac{2}{1-q}-3\right)
$$

The values of this scale $\delta_{\text {jump }} s$ are marked with an arrow in Fig. 21 for each chosen value of the bond activation probability $q$. These estimated values correspond correctly to the region of scales $\delta$ where the jump in the dependence of SFM on the bin size is present. 


\subsubsection{Percolation model in 3D}

The experiment al mult ifragmentation data was studied using a simple cubic lattice bond model containing about the same number of sites $\left(A=6^{3}\right)$ as nucleons in the fragmenting gold nucleus [35, 36]. The evolution of the fragment size distribution $N(s)$ in the bond percolation models depends solely on the value of the bond parameter $q$, which is a control parameter of the partition distribution. Critical point of percolation in $3 \mathrm{D}$ model is found at $q_{c}=0.25$ in the infinite $(A \longrightarrow \infty)$ network. At this point, the percolation cluster spans over a whole network and $N(s)$ exhibits self-similarity at all scales. In the finite network, the approximate self-similarity of $N(s)$ and the intermittent pattern of fluctuations is seen in the narrow range of bond parameters $0.21<q \leq 0.27$ with maximal slopes at $q \simeq 0.23$ ( see Fig. 22). At $q=0.28$ the intermittency exponents vanish.

One cannot parametrize the multifragmentation data in terms of $q$-values. In ref. $[35,36]$ an intermittent behaviour was found for events selected either according to the number of heavy fragments $\left(N_{f r} \geq 2,3,4, \ldots\right.$ of charge $s_{f_{r}} \geq 3$ ) or the total multiplicity of fragments $28<\check{M}<56 \quad(0.35<m<0.7$ in the scaled units $m \equiv \tilde{M} / \dot{M}_{\max }$, where $\dot{M}_{\max }=79$ is the maximal possible number of fragments). To simulate the experimental situation, the percolation calculation is performed with the bond parameter $q$ randomly distributed between 0 and 1 . The size of the heavy fragment $s_{f r}$ is chosen here to be bigger than $4\left(s_{f r} \geq 5\right)$ in order to achieve a closer correspondence of calculated mass distributions with the experimental charge distributions, for which $s_{f_{r}} \geq 3$. A heavy fragment in both cases is defined as a cluster with mass ( charge) greater than the mass ( charge) of the $\alpha$-particle. In Fig. 23 we compare the SFM testing the power-laws (4.19) for the Au - emulsion interaction [43] and the random percolation model with the similar criteria for selecting the events $\left(N_{f r} \geq 2,3\right)$ in both cases. Apart from the value of the non-universal value of the SFM in largest bin which differs in these two cases, the experimental and calculated curves are remarkably similar. They show also a similar small deviations from the linearity for large values of $\ln F_{2}$.

Percolation model in higher dimensions $(D>1, D \neq \infty)$ cannot be solved analytically. However, the same mechanism of the clustering phenomenon as in 1D operates, and the same three contributions to the correlations are present, namely:

- the upper mass bound $\left\langle n_{j} n_{k}\right\rangle=0$ for $j+k>A$, which is numerically not important,

- the repulsive correlations arising from the fact that the second fragment is chosen in a smaller system $(A-j)$,

- the attractive correlations arising from the configurations where the two fragments have one or more common empty links in their perimeters.

For simplicity let us consider the correlations between one large fragment of mass $s$ and one fragment of mass 1 i.e. $\left\langle n_{s} n_{1}>\right.$. The mean number of clusters of mass $n_{s}$ is [37] :

$$
<n_{s}>=A \sum_{t} g_{s t} q^{s}(1-q)^{\ell}
$$


where $g_{s t}$ is the mumber of elusters with size and perimeter t per lattice site. Taking inte account only the configurations where the two chusters have at most one common link in the perimeter one obtains :

$$
r_{s 1} \sim \frac{1}{A}\left(\frac{t}{1-q}-s-t\right)
$$

The perimeter $t$ takes in the percolating point the form [136] :

$$
t=s(1-q) / q+\text { const } s^{\sigma} \text {. }
$$

where the second term is not present below the critical point $q_{c}$. From (4.27) and (4.28) one obtains :

$$
c_{s 1} \sim \frac{\text { const } q}{A(1-q)} s^{\sigma} .
$$

The appearance of those attractive correlations is a surface effect linked with the appearance of the excess perimeter $\sim$ const $s^{\sigma}$ at the percolating point. The magnitude of those correlations decreases with the increasing size A of the system though the details of this dependence are not known. In the next section, we shall present the random cascading model, where the $A$-dependence of the intermittent behaviour can be understand analytically. Above the percolating point the number of fragments of "finite" size decreases and consequently the correlation effects between those fragments become less important. This is not only true for the fragments of size s and 1 but for any two fragments of "finite" size. The decrease of the total number of fragments in flavour of the increasing mass of the infinite cluster is responsible for the disappearance of the intermittency signal above the percolating point. The above discussion explains qualitatively the variation of SFMs with $q$ around $q_{c}$ as seen in Fig. 23 .

In the finite system, there exist several different types of correlations both attractive and repulsive, which contribute to the fluctuations in the fragment size distribution. The fragment-size distribution of the percolation in $3 \mathrm{D}$ close to $q_{c}$, exhibits the intermittent pattern of fluctuations which shows up on top of the "background" of other non-intermittent fluctuations. The mixing of the events corresponding to different $q$ values enhances in general the intermittency effect, providing that the "critical" events for $q \simeq q_{c}$ are contained in this sample [35, 36, 137]. It has been revealed, that the intermittency signal is not much sensitive to the distortions generated by the detector efficiency. On the contrary, it may be significantly reduced if the pre-equilibrium particles, simulated in $3 \mathrm{D}$ percolation by a gaussian source, are not carefully separated out[137] .

In this context, one should also mention the study of critical behaviour in classical molecular dynamics by Latora et al. [138]. In this dynamical model, the disassembly of hot nuclei at various temperatures has been studied with the aim of finding a relation between the power-law form of the fragment-size distribution and the intermittent pattern of fluctuations in the fragment-size distribution. Fig. 24 shows the mass distributions and SFMs for class of events characterized by a different initial temperature. At the temperature $T \simeq 5 \mathrm{MeV}$, the fragment-size distribution exhibits the power-like shape which is accompanied by a critical fluctuations of the intermittent type. This example demonstrates that, it may be extremely interesting to relate the 
values of the intermittency exponents to the dynamical conditions of the collision and a subsequent fragnentation process as well as to the various degree of "violence" of the collision as characterized e.g. by the excitation energy, impact parameter, multiplicity of fragments and least but not last to the size of the fragmenting system.

Similar analysis were performed in a statistical model of nuclear fragmentation. In this model, consisting of the metropolis sampling of the microcanonical fragmentation partitions, intermittent fluctuations of the fragment distributions were found [115].

\subsection{Cascade models}

The $\alpha$ - model of self-similar cascading [2] (sect. 3.2), could be viewed as a very simple picture of the multiparticle production. Indeed, the cascade models of particle production should also lead to the strong correlations for particles close in phase-space. This is due to the fact that in such models the particle production probability is enhanced in the vicinity of the parent particle. The $\phi^{3}$ branching model was first analyzed by Hwa [139], who found scaling correlations. This was confirmed in the numerical simulations [140] for several types of splitting functions. The phenomenological models of particle production also show some correlations which build up during in the cascade process [141, 142]. One of such well defined models where the multiparticle correlations can be studied is the perturbative QCD cascade. However, the comparison with the data on the observed final hadrons, requires the assumption of the local parton hadron duality to be true down to very small scales. We have seen in sect. 3.4.4 that the hadronization can change dramatically the correlation functions at the hadronization scale. Similar observations were made in a simple lattice gas model [99].

\subsubsection{QCD cascade}

The studies of scale-invariant correlations in the perturbative QCD started few years ago in the dipole model [143], where fractal dimensions of the gluon distributions in the $x$-space were calculated. The multiparton correlations were studied also by several groups in the perturbative gluon cascade $[5,107]$. In the double logarithm approximation (DLA) and in the high energy limit, where the cut-off is not important, the perturbative QCD cascade shows intermittent behaviour for the case of fixed coupling constant, and a scaling with scale dependent intermittency index for the case of running coupling constant. The intermittency indices of the QCD cascade in the angular variables are $[5,107]$ :

$$
\nu_{i}=(i-1)-1 / 2(i-1 / i) a,
$$

where $a^{2}=6 \alpha_{s} / \pi$ for fixed coupling constant, and

$$
a^{2}\left(P_{T}\right)=\frac{\beta^{2}}{\ln \left(P_{T} / \Lambda\right)}
$$

for running coupling constant. $\Lambda$ in the above expression denotes the QCD scale. 
Wosiek and Ochs calculated recursively the many parton correlations in the DIAA [107]. The many-parton distributions can be calculated from the generating functional $Z_{P}(u)$ :

$$
\rho_{n}\left(p_{1}, \ldots, p_{n}\right)=\frac{\delta^{n} Z(u)}{\delta u\left(p_{1}\right) \ldots \delta u\left(p_{2}\right)} .
$$

In the DLA, the generating functional obeys the following master equation :

$$
Z_{P}(u)=\exp \left(\int_{\Gamma_{P}(K)} \mathcal{M}_{P}\left(K^{\prime}\right)\left(u\left(K^{\prime}\right) Z_{K^{\prime}}(u)-1\right) d^{3} K\right)
$$

where $P$ denotes the momentum of the parent parton and $\Gamma_{P}\left(K^{\prime}\right)$ is the phase space of the integration which in the DLA approximation takes the form :

$$
\Gamma_{P}(K)=\left\{K: K<P, \Theta_{K P}<\Theta, K \Theta_{K P}>Q_{0}\right\},
$$

where $Q_{0}$ is a cut-off parameter. This leads to the recursive relation for the $n$-particle densities :

$$
\rho_{n(P)}\left(k_{1}, \ldots, k_{n}\right)=d_{n(P)}\left(k_{1}, \ldots, k_{n}\right)+\int_{\Gamma} d^{3} K \rho_{n(K)}\left(k_{1}, \ldots, k_{n}\right),
$$

where in the case of the gluonic cascade the Born emission probability is :

$$
M_{P}(K) d^{3} K=a^{2} \frac{d K}{K} \frac{d \Theta}{\Theta} \frac{d \phi}{2 \pi}
$$

and $\Gamma$ is integration region taking into account the angular ordering in the double logarithm approximation. The term $d_{n}\left(k_{1}, \ldots, k_{n}\right)$ describes the contribution from the lower order densities. In the leading order the partons are angularly ordered, i.e. a parton with momentum $K$ can emit further partons only inside the cone around $K$ limited by $P$. There is also an infrared cut-off regularizing the singularity of the emission probability for collinear partons.

The correlation functions take the simplest form in the angular variables $\Theta_{P K}, \phi_{i}$ with the momentum dependence integrated over. The integrals can be calculated in the pole dominance approximation, which uses the fact that the main contribution comes from the region where the momenta of two partons are almost parallel.

By angular integrations one can obtain the corresponding cumulant moment and scaled cumulant moments in a cone with position $\theta$ and opening angle $\delta$. This gives respectively :

$$
\mathcal{K}_{n}<N>^{n} \simeq a^{n / 2-1}(P \theta)\left(\frac{P \theta}{\Lambda}\right)^{2 n a(P \theta)}\left(\frac{\theta^{2}}{\delta^{2}}\right)^{-1-(1 / 2)(n-1 / n) a(P \theta)}
$$

for the cumulant moments and :

$$
\mathcal{K}_{n} \simeq \frac{1}{a(P \theta)}\left(\frac{\theta^{2}}{\delta^{2}}\right)^{n-1-(1 / 2)(n-1 / n) a(P \theta)}
$$

for the scaled cumulant moments. In the case of the constant coupling, for angles much larger than the cut-off $\theta>Q_{0} / P$, one obtains the power-law in two particle correlation :

$$
C_{2}(\delta) \simeq \frac{a}{2 \delta}\left(\frac{\delta}{Q_{0} / P}\right)^{2 a}\left(\frac{\theta}{\delta}\right)^{a / 2}
$$


The corresponding reduced correlations take the form :

$$
c_{2}(\theta) \simeq\left(\frac{\theta}{\delta}\right)^{a / 2}
$$

Clearly we can identify a scale-invariant regime in the two-particle correlations. This is true for high energy and angles much larger than the cut-off. The running coupling constant changes this result, since then the intermittency index becomes "scaledependent". Also as we approach the cut-off the correlations are changed. Generally, the correlation functions can be written as a function of one scaling variable $\epsilon=\ln (\Theta / \theta) / \ln (P \Theta / \Lambda)$. This situation is similar the discussion of the self-similar cascade with an infrared cut-off [97], where the presence on the cut-off required the use of universal multiscaling variables of the form :

$$
\frac{\ln (\Delta Y / \delta y)}{\ln (E / e)}
$$

where $E$ is the total energy and $e$ is the cut-off. In QCD the total available energy in the jet is $P \Theta$ and the QCD scale is the effective cut-off in the cascade. The running coupling constant can also be expressed by the universal scaling variable $\epsilon$ :

$$
a \simeq \sqrt{\alpha_{s}} \simeq \sqrt{\frac{1}{\epsilon}} .
$$

Thus in the variables $\sqrt{\ln (1 / \epsilon)} \ln \mathcal{K}_{i}$ and $\ln (\delta)$, one should observe a straight line, i.e. a scaling behaviour. If one wants to take into account the cut-off modifications of the correlations, one should use the multiscaling variables (eq. 3.43). Then, the universal multiscaling variables for QCD cascade taking into account both the running coupling constant and the cut-off read :

$$
\begin{aligned}
& \frac{\ln \left(\mathcal{K}_{n}\right)}{\sqrt{\ln (1 / \epsilon)}}, \\
& \frac{\ln (\Theta / \delta)}{\ln (1 / \epsilon)}
\end{aligned}
$$

In these variables one observes a scaling regime and a universal deviations from the scaling for any value of the reduced cut-off $\epsilon[107]$.

\subsubsection{Fragmentation-inactivation binary model}

This model of binary sequential fragmentation has been introduced recently [120] as a generalization of a population balance approach [144]. As in previous works [145], the physical breakup mechanisms are approximated by breakup rates, and this leads to the well-known linear rate equation for fragmentation $\{146]$. But there is no end for such a process, except by stopping it arbitrarily at a certain time. Since in most physical situations, fragmentation must stop gradually because of some dissipative phenomena, we simulate this additional hidden process by an inactivation rate. At this stage, the breakup and inactivation rates are given a priori and should be calculated 
by some microscopic theory in each realistic situation. In particular, this model is a purely kinetic one and is not connected to any geometric features like in percolation for example. A typical sketch of the fragmentation - inactivation binary (FIB) process is shown in Fig. 25.

Let us define the state of the system by a set of two vectors : $\vec{m}, \vec{n}$ where $\vec{m}=$ $\left(m_{1}, m_{2}, \ldots\right)$ is the list of the number of active monomers, dimers, etc., and $\vec{n}$ is the same but for inactive clusters. At the beginning of the process, we have : $\vec{m}=$ $\vec{u}_{N}$ where $\vec{u}_{N}$ has zero components, except 1 at the $n^{t h}$ place, and $\vec{n}=\overrightarrow{0}$. At the end of the same process, we have: $\vec{m}=\overrightarrow{0}$ and we wish to know what $\vec{n}$ is. The initial condition will be denoted by subscript $N$ when needed.

Let us now look at the first event of the sequence : it is either a fragmentation $(N) \longrightarrow(i)+(N-i)$ or an inactivation $(N) \longrightarrow(N)^{*}$. Suppose that this first event was a fragmentation at a time $t_{1}$. We may then consider one state $\{\vec{m}, \vec{n}\}$ at a given time $t$ for the initial condition $i$, and another state $\left\{\vec{m}^{\prime}, \vec{n}^{\prime}\right\}$ at a time $t-t_{1}$ for the initial condition $N-i$, with the constraints : $\vec{m}=\vec{m}^{\prime}+\vec{m}^{\prime \prime}$ and $\vec{n}=\vec{n}^{\prime}+\vec{n}^{\prime \prime}$. This leads to the following equation for the change in the probability $w_{N}(\vec{m}, \vec{n} ; t)$ to get one state $\vec{m}, \vec{n}$ at the time $t$ :

$$
\begin{aligned}
w_{N}(\{\vec{m}, \vec{n}\} ; t) & =\delta\left(\vec{m}-\vec{u}_{N}\right) \delta(\vec{n}) e^{-\Phi_{N} t} \\
& +I_{N} \delta(\vec{m}) \delta\left(\vec{n}-\vec{u}_{N}\right) \frac{1-e^{-\Phi_{N} t}}{\Phi_{N}} \\
+ & \sum_{i=1}^{N-1} \int_{0}^{t} B_{i, N-i}\left[\begin{array}{l}
\sum_{\vec{m}^{\prime}+\vec{m}^{\prime \prime}}=\vec{m} \\
\vec{n}^{\prime}+\vec{n}^{\prime \prime}=\vec{n}
\end{array}\right. \\
& \left.w_{i}\left(\left\{\vec{m}^{\prime}, \vec{n}^{\prime}\right\} ; t^{\prime}-t\right) w_{N-i}\left(\left\{\vec{m}^{\prime \prime}, \vec{n}^{\prime \prime}\right\} ; t^{\prime}-t\right) e^{-\Phi_{N} t^{\prime}}\right] d t^{\prime} .
\end{aligned}
$$

where we have denoted :

$$
\Phi_{k}=I_{k}+\sum_{i=1}^{k-1} B_{i, k-i} .
$$

In the above equations $I_{k}$ is the probability per unit of time for a given cluster of size $k$ to inactivate, and $B_{i, k-i}$ is the probability per unit of time for such a cluster to breakup into one fragment of size $\mathrm{i}$ and another of size $k-i$. It is interesting to notice that this fragmentation is a random multiplicative process, since the probability $w_{N}$ on right-hand side of eq. (4.33) equals to some linear functional of products like $w_{i} w_{N-i}$.

The equations giving the average value of any quantity related to the size distributions are then easy to write. For instance, if $m_{k}$ and $n_{k}$ means the numbers of $k$ - clusters, active and inactive respectively, one finds the two sets of coupled linear differential equations [120] :

$$
\begin{aligned}
& \frac{d<m_{k}>}{d t}=-\Phi_{k}<m_{k}>+2 \sum_{i \geq k+1} B_{k, i-k}<m_{i}> \\
& \frac{d<n_{k}>}{d t}=I_{k}<m_{k}>
\end{aligned}
$$


In these equations, the initial size of the system has been taken equal to $N$. The brackets $\langle>$ mean the average over many independent events.

In most cases, the two kernels $B_{i, k-i}$ and $I_{k}$ are not directly available from the relevant microscopic theories, but they are related simply to other more clear quantities : the probability $p_{u}(k)$ that a cluster of size $k$ becomes unstable and the probability $f_{i, k-i}$ that, when it breaks up, one fragment has size $i$ and the other one has size $k-i$. In effect, one has :

$$
\begin{aligned}
B_{i, k-i} & \equiv f_{i, k-i} \sigma_{k} \\
I_{k} & \equiv \frac{\sigma_{k}}{p_{u}(k)-1},
\end{aligned}
$$

where the function $\sigma_{k}$ corresponds to the inverse of a typical fragmentation time for a $k$-cluster. Note that the sequence of the fragmentation cascades is independent of this latter function $\sigma_{k}$ which governs only the times at which the cascades occur. For the particular case : $I_{k}=0$, we recover indeed the linear rate equation, but if this inactivation kernel is positive, there is a non trivial asymptotic (for $t \longrightarrow \infty$ ) size-distribution. Taking this limit is a great simplification in this sort of equations. An integration of the equation (4.46) in time, gives the following backward recurrent equation :

$$
\frac{\Phi_{k}}{I_{k}}<n_{k}>=2 \sum_{i=k+1}^{N} \frac{B_{k, i-k}}{I_{k}}<n_{i}>+\delta(N-k)
$$

from which many features of the size-distribution can be found either analytically or numerically $[120,147]$. Maybe the most spectacular result is the power-law behaviour of this size-distribution for a large group of homogeneous fragmentation and inactivation kernels[148]. If , for example, $B_{i, j}=(i j)^{\alpha}$ and $I_{k}=I_{1} k^{\beta}$, then :

$$
<n_{k}>=\frac{c(n)}{k^{\tau}}
$$

when $\beta \leq 2 \alpha+1$ and $\alpha>-2$. If $\beta>2 \alpha+1$, then almost the whole mass is concentrated in one large cluster but the size-distribution of the remaining fragments is still well approximated by a power-law. The case where $\alpha<-2$ has not yet been investigated completely and will not be discussed in this review. In the region $\beta<2 \alpha+1$, the coefficient $c(N)$ is proportional to $N$ and this means that a finite proportion of the total mass is in the form of monomers, i.e. clusters of size $k=1$. On the contrary, as soon as $\beta=2 \alpha+1$, the coefficient $c(N)$ behaves like $N^{\tau-1}$ with a value of $\tau$ smaller than 2 and the relative proportion of the total mass of the monomers is zero in the limit of an infinite system.

It has been shown $[120,147]$, that the parameter $\left\langle n_{1}\right\rangle / N$, in the limit of infinite $N$, is an order parameter which is 0 in the $\infty$-cluster phase : $\beta>2 \alpha+1$, and is positive in the other phase : $\beta<2 \alpha+1$. The line which separates these two phases is a critical line for this fragmentation process. This behaviour has been seen first in the standard rate-equation model and is called the "shattering" transition [149]. The size-distribution fluctuations of such a model are suspected to be very interesting due to both the cascade structure of the model and its critical behaviour. 
Numerically, the scaled factorial moments show no intermittency in the shattered phase $(\beta<2 \alpha+1)$ but they show intermittency on the critical line. A typical example is shown in Fig. 26.

An analytical calculation of the SFMs in FIB model can be carried out in some particular points of the phase diagram $\alpha, \beta$. One is the choice : $B_{i, j}=1$ and $I_{k}=k \quad(\alpha=0, \beta=1)$ corresponding to $\tau=1$. It is on the transition line and an elementary derivation of the second horizontal scaled factorial moment $F_{2}$ in the limit when the total mass is large leads to [148]:

$$
\ln \left(\frac{F_{2}\left(\delta_{s}\right)}{\ln N}\right) \sim-\frac{2}{3}\left(\frac{\ln \delta_{s}}{\ln N}\right),
$$

where $\delta_{s} \equiv N / M$ is the width of each bin. Analogously as in sects. 3.4 .4 and 4.2.1, one can introduce the universal multiscaling variables :

$$
\begin{aligned}
G_{2}(\Theta) & =\ln \left(\frac{F_{2}\left(\delta_{s}\right)}{F_{2}(1)}\right) / \ln N \\
\Theta & =\ln M / \ln N
\end{aligned}
$$

in which the eq. (4.50) takes the usual form :

$$
G(\Theta)=f_{2} \Theta
$$

with

$$
f_{2}=\frac{2}{\ln N}
$$

Hence, the spectrum of fluctuations as measured oy SFMs, corresponds to an intermittent behaviour of the fluctuations with the intermittency exponent $f_{2}$ which $\log$ arithmically depends on the system size $N$. In other terms, one may conclude that the natural scaled variable appear here to be $\ln M / \ln N$ instead of $\ln M$. Notice, that for the same value of the exponent $\tau$ of the power-law cluster size distribution, the Mekjian model $(x=1)$ does not exhibit intermittency in SFMs. Thus, similarly as for the shattered phase of the FIB model, the power-law form of the cluster-size distribution is not yet sufficient for the appearance of intermittent fluctuations.

It is tempting to compare this result to Parisi - Frisch multifractal model of fully developed turbulence[98] . In a fluid at high Reynolds numbers $R$, the scaled energy spectrum $\ln \varepsilon / \ln R$ is a universal function of the scaled wavelength $\ln \lambda / \ln R$ and the Reynolds number is proportional to the total size of the fluid. In the same way, the cut-off parameter $\varepsilon \equiv 1 / N$ is related to the total size $N$ of the system and the good scaled variable is $\ln M / \ln N$. This is also analogous to the low-density cut-off in the random cascading picture of the multiparticle production [97] as discussed in sect. 3.4.4.

Since the size-distribution is singular at the smallest masses (eqs. (4.50) - (4.52) correspond to the power-law fragment-size distribution with $\tau=1$ ), the horizontal factorial moment coincides with the corresponding vertical moment calculated for the first bin. 
Another choice of the kernels for which an analytical derivation can be done is : $B_{i, j}=1$ and $I_{k}=1 \quad(\alpha=0, \beta=0)$. This case lies inside the "shattering" phase, i.e. the quantity $\left\langle n_{1}\right\rangle / N$ tends to a finite value (here: $1 / 3$ ) as $N$ goes to infinity. The second horizontal SFM behaves like:

$$
\log F_{2} \sim \exp \left[2\left(-\ln \delta_{s}\right)\right] \quad,
$$

and does not show any scale-invariance. For $\log \delta_{s}>>1$ the value of $F_{2}$ is comparable with 1. Notice, that the two considered cases $\alpha=0, \beta=0$ and $\alpha=0, \beta=1$ in the shattering phase and on the transition line respectively, yield qualitatively similar behaviour of fluctuations as found in percolation below and at around the critical point (see sect. 4.1.3 and Fig. 23).

The Au - fragmentation data of ALADIN for the collision of $A u$ nuclei at $600 \mathrm{MeV} / u$ on different targets[150] have been successfully reproduced by choosing the kernels at the critical line of the FIB model[151]. Different choice of parameters $\alpha, \beta, I_{1}$, corresponding to different temporary evolutions, turned out to give the same results for the multiplicity spectra, providing the critical exponent $\tau(\tau=1.85)$ of the powerlaw fit of the fragment-mass distribution is kept fixed[151]. In this case the fluctuations in the fragment-size distribution, as measured by SFMs, exhibit an intermittent pattern for all those "equivalent" parameters $\alpha, \beta, I_{1}$. Notice in Fig. 26 , that intermittency exponents are almost identical for "equivalent" sets of the parameters.

\subsection{Bose-Einstein correlations}

The importance of the Bose-Einstein effect in the intermittency studies was recognized very early $[152,123]$ The experimental results in $3 \mathrm{D}$ show that the main effect is due to the correlation between like-charge particles. Fig. 27 shows the two-particle correlation function as observed in the $p-\bar{p}$ reaction by the UA1 Collaboration, clearly showing that the Bose-Einstein correlations are very strong at small scale and follow a power-law. This means that the Bose-Einstein interference is mainly responsible for the observed strong correlations at small scales. The phenomenological implications of the above fact are twofold [153]. First, because even at the highest presently achieved resolution we still observe a rise in the two-particle correlations, the size of the source must be quite large. Since the momentum scales presently studied are about $30 \mathrm{MeV}$, we expect a source of the extension of at least $6-7 f \mathrm{~m}$. Second, the power-law dependence of the two-particle correlation on the momentum difference at small momentum requires a power-law behaviour of the source density at large scales. It can be either an extended power-law tail in the density profile of the emitting source or an extended fractal source.

\subsubsection{Small-scale Bose-Einstein correlations}

The presence of the power-law Bose-Einstein correlations at small scales requires very specific shape fluctuations in the long-range region $[154,155]$. The $n$-particle 
interferometry reduced densities can be written as:

$$
D_{n}\left(k_{1}, \ldots, k_{n}\right)=\frac{\int d x_{1} \ldots \int d x_{n}\left(\sum_{p e r} \exp \left(i\left(x_{1} p_{a_{1}}+\ldots+x_{n} p_{a_{n}}\right)\right) d_{n}\left(x_{1}, \ldots, x_{n}\right)\right.}{n !\left(\int d x d_{1}(x)\right)^{n}}
$$

where $d_{n}$ is the $n$-point density of emitting sources and the sum runs over all the permutation of the indices $a_{i}$. The above formula supposes incoherent emission and neglects the final state interaction. In the case of uncorrelated emission in space-time:

$$
d_{n}\left(x_{1}, \ldots, x_{n}\right)=d_{1}\left(x_{1}\right) \ldots d_{n}\left(x_{n}\right) .
$$

In this case, the $n$-particle cumulant can be written using the Fourier transform of the source density $d_{1}(x)$ in the following way[28] :

$$
d_{n}\left(k_{1}, \ldots, k_{n}\right)=\sum d_{1}\left(k_{1}-k_{a_{1}}\right) \ldots d_{1}\left(k_{n}-k_{a_{n}}\right),
$$

where the sum is over all permutations of the indices $a_{i}$ with $a_{i} \neq i$. If the source density $d_{1}(x)$ has a power-law tail :

$$
d_{1}(x) \simeq x^{\gamma-D}
$$

its Fourier transform shows also a power-law in some range of small momenta [28] :

$$
d_{1}(k) \simeq|q|^{-\gamma} .
$$

Thus, we obtain a relation between the power-law tail in the source density and the power-law in the two-pion Bose-Einstein correlations which are given in terms of the Fourier transform of the source density :

$$
C_{2}(q) \simeq|q|^{-2 \gamma}
$$

The formula (4.57) shows that the higher order cumulants are then expected also to have a power-law dependence on the rescaling of momenta with an index :

$$
\nu_{i}=i \gamma
$$

Thus, the intermittency indices grow linearly with the increasing order of the correlations. There is not much experimental data concerning the higher order cumulants. Only the NA22 group measured the third order cumulant, with the preliminary result [28] :

$$
\nu_{3}=2 \nu_{2}
$$

This does not follow the relation (4.61), and would mean that there are some additional correlations between like-charge pions. This could be due to the correlated emission of pions from the source. An example of such a correlated source was discussed in sect. 4.1.1 for the case of ultrarelativistic nuclear collisions. In the next section we shall discuss some implications of this scenario of spatio-temporal intermittency for the Bose-Einstein correlations. 


\subsubsection{Large-scale Bose-Einstein correlations}

The hypothesis of the spatio-temporal intermittency rises the question if such a strong correlation between the emitting sources in space-time could be observed in Bose-Einstein correlations between the produced particles [106].

The pion interferometry can measure the possible underlying correlations of the emitting sources and its correlation length. For sources correlated in space-time, eq. (2.11) takes the form $[106]$ :

$$
D_{2}(k)=\frac{\int d^{4} x_{1} \int d^{4} x_{2} d_{2}\left(x_{1}, x_{2}\right)\left(1+\cos \left(\left(k_{1}-k_{2}\right)^{\mu}\left(x_{1}-x_{2}\right)_{\mu}\right)\right)}{\left(\int d^{4} x d_{1}(x)\right)^{2}} .
$$

If the number of sources is large, then :

$$
\int d^{4} x_{1} \int d^{4} x_{2} d_{2}\left(x_{1}, x_{2}\right) \sim\left(\int d^{4} x d_{1}(x)\right)^{2} .
$$

We calculate the two-pion correlation function assuming a spherical static source in three-dimensions. The source distribution function is in this case :

$$
N \frac{d_{1}\left(\overrightarrow{R_{1}}\right) d_{1}\left(\vec{R}_{2}\right)}{\left|\overrightarrow{R_{1}}-\overrightarrow{R_{2}}\right|^{\nu_{2}}}
$$

with the source density $d_{1}(\vec{R})=\exp \left(-\lambda \vec{R}^{2}\right)$. The parameter $\lambda=2 /\left(\pi<R>^{2}\right)$ is given by the mean radius $\langle R\rangle$ and can be related to the radius of a sphere $R_{s p h}$ with the same mean radius :

$$
\lambda \sim \frac{1.13}{<R_{s p h}>^{2}} .
$$

We take $R_{s p h}=3.5 \mathrm{fm}$ as in the central S-Em collisions. Then the equation for $D_{2}(\vec{k})$ simplifies to :

$$
D_{2}(\vec{k})=\frac{\int d^{3} \vec{R} \exp \left(-\lambda \vec{R}^{2} / 2\right) \cos (\vec{R} \vec{k}) /|\vec{R}|^{\nu_{2}}}{2 \pi \Gamma\left(\frac{3-\nu_{2}}{2}\right)(2 / \lambda)^{\left(3-\nu_{2}\right) / 2}}+1
$$

The results for $\nu_{2}=1.0,0.5$ and $\nu_{2}=0$ (uncorrelated sources) are plotted in Fig. 28. The different distribution are indistinguishable from the case of the randomly distributed sources according to the density $d_{1}(\vec{R})$. The emission from correlated but not necessarily fractal sources, gives distribution function corresponding to an effectively smaller emitting region. In other words, if the sources are correlated then the radius of the interaction region is actually larger than the measured one, but still the effect is very small. The effect of correlated sources can be compensated by a change of about $15 \%$ of the radius of the interaction region (Fig. 28), and hence it cannot serve as a signal of the spatio-temporal intermittency. The difference between correlated and uncorrelated sources becomes substantial (Fig. 28) only in the region of large momentum difference of the pair of pions. This behaviour for large momenta reflects the presence of a singularity for small separation of sources (eq. 4.64). The signal of the singularity in this region of momenta would be probably difficult to disentangle from the background which is present in experimental studies of the correlation function. 
Its magnitude should be larger if the pion emission would take place on a surface. In this case, the effect of a correlated (fractal) distribution of sources could be better seen even in the Fourier transformed quantity such as the distribution function $D_{2}$ (Fig. $29)$.

\subsubsection{Bose-Einstein correlations and intermittency}

Although, the experimental results of several groups $[56,58,63,69]$, seems to confirm that the Bose-Finstein correlations are mainly responsible for the small scale correlations, the problem is not yet fully understood. The main questions are, why is the source emitting pions so large and why has it a power-law behaviour. As noticed by Fiatkowski, the intermittency indices for the second order cumulant are the same for different reactions and energies. This would mean that there exist some preferred source shape and possible correlation structure which is universal for different reactions. The mechanism of this possible self-organization is however not clear.

It seems, if the relation (eq. 4.62) found by the NA22 group is confirmed, that in order to reproduce the data the source should not only have a power-law density profile but also some space-time correlations between pion emitting points. The correlations should be present on large scale in space, so that after Fourier transforming they would give a power-law at small momenta. The presence of such strong power-law correlations at large scales is difficult to understand, unless the time scales involved in their creation during the reaction are also large. Indeed, simply the causality constraint requires a time scale at least of the order of the space scale involved and probably in order to build up such strong correlations a longer time is needed.

On the other hand, the correlations between emission points at small scales in space should be observable on large scales in momenta. However, the numerical illustration of this effect presented in the previous section shows that it would be very difficult to disentangle those large-momenta Bose-Einstein correlations from the background and other long-range correlations. If this could be observed, it would be an important confirmation of the correlated structure of the emitting source. In ultrarelativistic collisions, some part of those spatio-temporal correlations could be seen as fluctuations in rapidity distributions (sect. 4.1.1). If such strong rapidity fluctuations will be observed at RHIC energies and, at the same time, one will observe extended powerlaw tails in the Bose-Einstein correlations (sect. 4.3.2), then this will be a strong indication for a second-order phase transition in ultrarelativistic collisions. However, if the systems follows a hydrodynamical evolution in the stable regime, the fluctuations at small scales in space-time are rapidly damped (sect. 4.1.1).

It was noticed very long time ago by Grassberger, that the resonance decays could modify the Bose-Einstein correlations [156]. Indeed, the pions coming from decay of the resonances would show a different interference picture as the pions directly emitted from the source. As the resonance can propagate outside the source before its decay, the pions coming from its decay will indicate a larger source. This phenomenon could explain the first feature of the observed Bose-Einstein correlations at small scales , i.e. the presence of a large source even in the collisions of the elementary particles. It is however not clear whether the distribution of the resonance decay times, or more precisely decay points, follows at least approximately a power-law. 


\section{Closing discussion}

Several experimental groups analyzed the fluctuations in the particle distributions at small distances in the rapidity or, more generally, in the phase-space. The experimental results of the $2 \mathrm{D}$ and $3 \mathrm{D}$ analysis show a growth of the fluctuations with the resolution. This kind of behaviour is similar to the one observed, for example, in the classical turbulence or in the chaotic attractors and motivates the very attractive hypothesis that similar multifractal structures may be present in the multiparticle dynamics. The presence of such fractal multiparticle distributions should be manifested by a power-law increase of the SFMs with the resolution for small phase-space cells. This concept was at the origin of a big activity in this field, resulting in a good phenomenological understanding of the features of such self-similar structures. Similar behaviour was observed in a very different regime of excitation energies, in nuclear reactions leading to the fragmentation of a composite system into several intermediatemass fragments. There, the distributions of fragments produced in a disassembly of hot residual nucleus were studied. For some classes of events in nuclear multifragmentation, self-similar fluctuations in fragment distributions are also observed.

In the approach proposed by Bialas and Peschanski, the particle production is viewed as a multiplicative random cascade. The mechanism of the self-similar cascade could reproduce the basic features of the multiparticle final state $[1,2]$. The most important prediction of the cascade model (the $\alpha$-model) is the scaling of the SFM with the resolution. This simple cascading mechanism explains how the multiparticle distributions of a very special, self-similar type are build up.

As we already mentioned, the analysis presented in this work which was motivated by the analogy with classical multifractals, was based mainly on the assumption of the existence of the scale-invariant multiparticle correlations. The dimensional projection removes the singularities from the full-dimensional multiparticle distribution, leading to the experimentally observed flattening of the dependence of the SFMs on the bin width in rapidity. This is the reason for which the scaling behaviour should be observed in 3D. To deal with the difficult problem of calculating the correlations in very small cells in $3 \mathrm{D}$, the method of correlation integral was proposed [4]. In this method, several ways of estimating the integral of the correlation functions from the experimental multiparticle distributions were elaborated and several experimental groups presented already results of this analysis. From these results the following salient features can be listed :

- All experiments show an increase of the fluctuations at small scales in the distribution of particles produced in the collision. The same is true for some classes of nuclear multifragmentation events.

- The fluctuations are strongest in the $3 \mathrm{D}$ data, and smaller in the $1 \mathrm{D}$, and $2 \mathrm{D}$ distributions. This is consistent with the dimensional projection reduction of the fluctuations.

- In the 3D data, a scaling correlation was identified in the two-particle distributions, with a similar intermittency exponent for several reactions. 
- The 3D correlations at small scales are dominated by the Bose-Einstein correlations, with a power-law dependence on the resolution.

- It is not yet clear whether some nontrivial, possibly scale-invariant correlations exist between unlike-charge particles.

- The fluctuations observed in the reactions between heavy nuclei are more important than expected from a superposition of independent sources corresponding to elementary collisions. This implies that the observed correlations are due to the collective effect.

Several standard models were confronted with this new kind of observables. The conclusion is that the basic effect can be reproduced if the Bose-Einstein correlations and the known processes and experimental misindentifications are included in the LUND and FRITIOF models. The models cannot however reproduce the detailed dependence on the cuts in transverse momentum and multiplicity. Thus, the first hope that the intermittency phenomenon would learn us about the multiparticle production mechanisms, and help us to disentangle between different models is not so simple to realize. It is true that we do not understand why such strong and scale-invariant BoseEinstein correlations are observed, but this kind of correlations can be included in the present models only in a phenomenological way. Besides the Bose-Einstein type of correlations two other sources of small scale fluctuations are discussed: the self-similar cascade and the second-order phase-transition.

The cascade structure of the perturbative QCD was recently analyzed, showing that scaling correlations can be expected for partonic distributions[5]. These very interesting works show that one can relate the strong coupling constant to the intermittency indices of the partonic distributions. In the experiment, it is however difficult to separate the correlations due to the cascade production mechanism from other type of correlations and Bose-Einstein interference. From the theoretical point of view, there is also a question how to relate the partonic distribution to the hadron distribution at very small scales. The problem of the infrared behaviour is already present at the level of the partonic cascade, where the presence of the infrared cut-off changes the correlations functions. These changes can be calculated, at least as far as one believes in predictions of the perturbative QCD near the cut-off. However, the influence of the hadronization process in the present models is in fact not known. Actually, it depends on parameters of the models and specific implementation. Some arguments can be given, which show that the hadronization process can change dramatically the fluctuations and, more precisely, it increases the fluctuations at small scales $[97,99]$. The studies of the very interesting question of the limits of validity of the local parton - hadron duality, got new ideas and possibilities of experimental studies from the intermittency analysis.

A second order phase-transition in the ultrarelativistic collisions is expected to create scale-invariant fluctuations in the particle distributions. At present energies there is however no evidence for this kind of self-similar correlations. The theoretical approaches used the Ginzburg - Landau theories to describe the critical properties of the system and to calculate the intermittency indices. However, the relation must be found between the correlations in phase transition region and the experimentally 
observed correlations between the produced particles. Due to several nonideal effects. these correlations are strongly deformed.

The most intriguing open question appearing from the experimental observations is the unexpected nature of the Bose-Einstein correlations. In order to explain the data, a large coherent source should be present. Moreover, its density profile should be of a power-law type. It is not understood whether the resonance decay can form a source of this shape, resulting in strong power-law, like-sign particle correlations at small scales, or whether one should refer to some unknown mechanism which creates such structures in the emitting region. In the latter case, this mechanism should be universal, since it is observed in different reactions.

In the nuclear multifragmentation the observation of self-similar fluctuations was believed to be a strong clue in the search of the mechanism of the disassembly of hot nuclei. The relation was made with the percolation model where self-similar fluctuations are present around the percolation point, and are due to the geometrical features of the fragment formation. However, analogous fluctuation patterns were found in the statistical multifragmentation model and in the classical molecular dynamics simulations of heavy-ion collision at around the initial temperature, where the fragment-size distribution exhibits a power-law. Also in a quite general model of binary fragmentation and inactivation scaling fluctuations are found at the shattering transition line. This shows that the origin of the scale-invariant fluctuations in the fragment distributions is not yet known. Should it be of a geometrical origin as in percolation at the percolation threshold or should it be due to the specific competition of the scale-invariant fragmentation and inactivation kernels at the shattering transition line. Obviously, more experimental data are needed in order to pin down the nature of the nuclear multifragmentation process and its possible relation to a critical behaviour. New generation of $4 \pi$ - detectors will hopefully help in resolving this intriguing question.

One should mention, that there is no tradition in studying fluctuations in the cluster-size distribution and hence in the fragmentation process, even though these processes are related to different physical phenomena and some of them to critical phenomena, and scale-invariant inclusive cluster-size distributions are found in several of them.

The study of structures in the multiparticle production at small momentum scale is very interesting, and using the method of the SFMs or correlation integrals this study is now possible. These methods are a powerful tool of analyzing the multiparticle correlations at small scales. Using the these methods it was demonstrated for the first time that interesting phenomena occur at small distances in the momentum. The study of those small distance correlations could give insight into the hadronization mechanism, the structure of the interaction region, the mechanism of the nuclear multifragmentation and last but not least could be a signal of a phase transition. 


\section{Acknowledgements}

During our activity on this subject we have profited from many discussions and collaborations with Z. Burda, B.A. Li and in particular with A. Tucholski and we thank them very warmly. It is impossible to acknowledge the many influences on our present understanding and the ideas we have exposed from other theorists who contributed to our work via encouragement, discussion, opposition or alternative points of view. Among those from which we have gained mostly are: J. Alberty, A. Białas, P. Carruthers, X. Campi, D.H.E. Gross, H. Kirivine, R. Peschanski, J. Richert.

We thank B. Buschbeck and W. Kittel for providing the experimental results and A. Bonasera for allowing the mention of his results before publication.

This work was possible due to the financial support of the Commissariat à l'Énergie Atomique and Centre National de Recherches Scientifiques, Gesellschaft fur Schwerionenforschung in Darmstadt and Polish Atomic Energy Agency. The support of the Polish Government grant KBN 2.0204.91.01 is also acknowledged. P. Bozek much appreciates the hospitality extended by GANIL, where much of this work was carried out. 


\section{Bibliography}

[1] A.Białas and R.Peschanski, Nucl. Phys. B273 (1986) 703.

[2] A.Bialas and R.Peschanski, Nucl. Phys. B308 (1988) 857.

[3] D. Schertzer and S. Lovejoy, Selected papers from $4^{-{ }^{t h}}$ International Symposium on Turbulent Shear Flows, Karlsruhe University (1983), eds. L.J.S. Bradbury et al., Springer, 1984.

[4] P. Lipa, P. Carruthers, H.C. Eggers and B. Buschbeck, Phys. Lett. B285 (1992) 300.

[5] W. Ochs and J. Wosiek, Phys. Lett. B289 (1992) 159;

Ph. Brax, J-L. Meunier and R. Peschanski, Saclay Preprint SPhT/93-011;

Yu.L. Dokshitzer and I.M. Dremin, Nucl. Phys. B402 (1993) 139.

[6] B. Mandelbrot, J. Fluid. Mech. 62 (1974) 719;

U. Frisch, P. Sulem and M. Nelkin, J. Fluid. Mech. 87 (1978) 719;

Ya.B. Zeldovich et al., Zh. Eksp. Teor. Fiz. 89 (1985) 2061;

ibid. Usp. Fiz. Nauk 152 (1987) 3;

G. Parisi and U. Frisch, in Turbulence and Predictability in Geophysical Fluid Dynamics and Climate Dynamics, Proc. Int. School of Physics E. Fermi, Varenna, Italy, eds. M. Ghil, R. Benzi and G. Parisi, Course LXXXVIII (North-Holland, Amsterdam) 1985, p.84.

[7] W.A. Zajc, Nucl. Phys. A525 (1991) 315c.

[8] R. Hanbury-Brown and R.Q. Twiss, Nature 177 (1957) 27.

[9] G. Goldhaber et al., Phys. Rev. 120 (1960) 300;

G. Coccini, Phys. Lett. B49 (1974) 459;

G.I. Kopylov and M.I. Podgoretsky, Sov. J. Nucl. Phys. 15 (1972) 219; ibid. 19 (1974) 215;

G.I. Kopylov, Phys. Lett. B50 (1974) 572.

[10] For a recent review see D.H Boal, C-K. Gelbke and B.K. Jennings, Rev. Mod. Phys., 62 (1990) 553.

[11] K. Kolehmainen and M. Gyulassy, Phys. Lett. B180 (1986) 203.

[12] M.G. Bowler, Phys. Lett. B185 (1987) 205.

[13] H.C. Eggers, P. Lipa, P. Carruthers and B. Buschbeck, Phys. Lett. B301 (1993) 298.

[14] P.Carruthers and I.Sarcevic, Phys. Rev. Lett. 63 (1989) 1562;

P. Carruthers, H.C. Eggers and I. Sarcevic, Phys. Rev. C44 (1991) 1629.

[15] E.A. De Wolf, Acta Phys. Pol. B21 (1990) 611.

[16] L.Van Hove, Phys. Lett. B242 (1990) 485; Physica 147A (1987) 19.

[17] P. Bożek and M.Płoszajczak, Phys. Lett. B254 (1991) 502.

[18] K. Fiałkowski, W. Ochs and I. Sarcevic, Z. Phys. C54 (1992) 621. 
[19] P. Carruthers and Shih. Int. J. Mod. Phys.. A2 (1987) 14.t7.

[20] A.H. Mueller. Phys. Rev. D4 (1971) 150.

[21] T. Takagi, Phys. Rev. Lett. 53 (1984) 427.

[22] (UA5 Collab.), R.E. Ansorge et al., Z. Phys. C 37 (1988) 191;

(UA5 Collab. ), (i.J. Alner et al., Z. Phys. C 33 (1986) 33;

Phys. Rep. 154 (1987) 251.

[23] K. Fialkowski, Phys. Lett. B272 (1991) 139;

Z. Phys C61 (1994) 313.

[24] K. Fiałkowski, B. Wosiek and J. Wosiek, Acta Phys. Pol. B20 (1989) 639.

[25] A. Białas and M. Gazdzicki, Phys. Lett. B252 (1990) 483;

W. Ochs, Z. Phys. C50 (1991) 379.

[26] R. Peschanski, Proc. Santa Fe Workshop on Intermittency in High Energy Collisions, eds. F. Cooper, R.C. Hwa and I. Sarcevic, World Scientific, Singapore, 1991.

[27] (EMU01 Collab.) M.I. Adamovich et al. , Phys. Rev. Lett. 65 (1990) 412.

[28] A. Białas and K. Zalewski, Phys. Lett. B228 (1989) 155.

[29] P. Bożek and M. Ploszajczak, Phys. Lett. B271 (1991) 243.

[30] D. Seibert and S. Voloshin, Phys. Rev. D43 (1991) 119.

[31] (NA22 Collab.), V.V. Aivazyan et al., Phys. Lett. B258 (1991) 487.

[32] K. Haglin and D. Seibert, Phys. Lett. B 273 (1991) 211.

[33] R. Peschanski and J. Seixas, CERN Preprint CERN-TH-5903/90.

[34] H.C. Eggers, P. Lipa, P. Carruthers and B. Buschbeck, Phys. Rev. D48 (1993) 2040.

[35] M. Ploszajczak and A. Tucholski, Phys. Rev. Lett. 65 (1990) 1539.

[36] M. Ploszajczak and A. Tucholski, Nucl. Phys. A523 (1991) 651.

[37] D. Stauffer, Phys. Rep. 54 (1979) 1;

J.W. Essam, Rep. Prog. Phys. 43 (1980) 835.

[38] M.E. Fisher, Physics(N.Y.) 3 (1967) 255.

[39] J.P. Bondorf et al. , Nucl. Phys. A443 (1985) 321; ibid. A448 (1986) 753;

D.H.E. Gross et al., Z. Phys. A309 (1982) 41;

J. Randrup and S.E. Koonin, Nucl. Phys. A356 (1981) 223; ibid. A474 (1987) 173.

[40] X. Campi, J. Phys. A Math. Gen. 19 (1986) 917; Phys. Lett. B208 (1988) 351.

[41] Y.-T. Cheng, M.-A. Nicolet and W.L. Johnson, Phys. Rev. Lett. 58 (1987) 20;

E. Louis, A.G. Marti and F. Guinea, Nucl. Inst. and Meth. B18 (1987) 4;

K.B. Winterbon, H.M. Urbassek, P. Sigmund and A. G. Marti, Phys. Scripta, 36 (1987) 689.

[42] G. Bardos and F. Kun, Phys. Lett. A165 (1992) 401.

[43] P. Freier and J. Waddington, Phys. Rev. C31 (1985) 888.

[44] M. Ploszajczak, A. Tucholski and P. Bożek, Phys. Lett. B262 (1991) 383.

[45] P.L. Jain, G. Singh and M.S. El-Nagdy, Phys. Rev. Lett. 68 (1992) 1656.

[46] G. Singh, A. Mukhopadhyay and P.L. Jain, Z. Phys. A345 (1993) 305.

[47] D. Ghosh, P. Ghosh, J. Roychowdhury, A. Deb and J. Roy, Phys. Rev. C47 (1993) 1120.

[48] E.A. De Wolf, I.M. Dremin and W. Kittel, Antwerp Univ. Preprint HEN-363 (1993).

[49] B.A. Li, Phys. Lett. B292 (1992) 246; ibid. B300 (1993) 14;

B.A. Li, Phys. Rev. C.47 (1993) 693. 
[50] B.A. Li and M. Ploszajczak, Phys. Lett. B317 (1993) 300.

[51] (TASSO Collab.), W.Braunschweig et al., Phys. Lett. B231 (1989) 548.

[52] (DELPHI (ollab.). P. Abreu et al., Phys. Lett. B247 (1990) 137.

[53] (ALEPH Collab.), D. Abbaneo et al. , CERN Preprint (1991) CERN-PPE/91-121.

[54] (OPAL Collab.), M.Z. Akrawy et al., Phys. Lett. B262 (1991) 351.

[55] (CELLO Collab.), H.-J. Behrend et al. , Phys. Lett. B256 (1991) 97.

[56] (DELPHI Collab.). A. De Angelis, Mod. Phys. Lett. A5 (1990) 2395;

A. De Angelis and N. Demaria, Proc. Ringberg Workshop on Multiparticle Production.

Ringberg Castle, Germany, (1991), eds. R.C. Hwa, W. Ochs and N. Schmitz, World

Scientific, Singapore, 1992;

P. Abreu et al., Nucl. Phys. B386 (1992) 471.

[57] I.Derado, G. Jancso. N. Schmitz and P. Stopa, Z. Phys. C47 (1990) 23.

[58] I. Derado, G. Jancso and N.Schmitz, Z. Phys. C56 (1992) 553.

[59] L. Verluyten et al., Phys. Lett. B260 (1991) 456.

[60] (NA22 Collab.), I.V.Ajinenko et al., Phys. Lett. B222 (1990) 306; ibid. B235 (1990) 373.

[61] (NA22 Collab.), N.M. Agababyan et al., Phys. Lett. B261 (1991) 165.

[62] (UA1 Collab.), C.Albajar et al., Nucl. Phys. B345 (1990) 1.

[63] (UA1 Collab.), N. Neumeister et al., CERN Preprint CERN-PPE/93-152.

[64] J.B. Singh and J.M. Kohli, Phys. Lett. B261 (1991) 160.

[65] (NA22 Collab.), F. Botterweck et al., Z. Phys. C51 (1991) 37.

[66] (KLM Collab.), R. Hołyński et al., Phys. Rev. Lett. 62 (1989) 733.

[67] (KLM Collab.), R. Hołyński et al., Phys. Rev. C40 (1989) 2449.

[68] (EMU01 Collab.), M.I. Adamovich et al. , Z. Phus. C49 (1991) 395.

[69] (NA35 Collab.), J. Bächler et al., MPI Preprint MPI-PhE/93-27.

[70] P.L. Jain and G. Singh, Phys. Rev. C44 (1991) 854.

[71] E. Gładysz-Dziaduś, Mod. Phys. Lett. A4 (1989) 2553.

[72] K. Kadija and P.Seyboth, Phys. Lett. B287 (1992) 363.

[73] R. Peschanski, Nucl. Phys. B327 (1989) 144;

A. Białas and K. Zalewski, Phys. Lett. B238 (1990) 413;

Ph. Brax and R. Peschanski, Nucl. Phys. B346 (1990) 65;

ibid. B353 (1991) 165.

[74] P. Brax and R. Peschanski, Phys. Lett. B253 (1991) 225.

[75] C. Meneveau and K.R. Srennivasan, Phys. Rev. Lett. 59 (1987) 1424;

P. Lipa and B. Buschbeck, Phys. Lett. B223 (1989) 465.

[76] J.Wosiek, Acta Phys. Pol. B19 (1988) 863.

[77] B. Bambach, J. Fingberg and H. Satz, Nucl. Phys. B332 (1990) 629;

S. Gupta, P. Lacock and H. Satz, Nucl. Phys. B362 (1991) 583.

[78] H. Satz, Nucl. Phys. B326 (1989) 613.

[79] I. Novak, Bratislava Preprint B-3837 (1989).

[80] Z. Burda, J. Wosiek and K. Zalewski, Phys. Lett. B266 (1991) 439;

Z. Burda, K. Zalewski, R. Peschanski and J. Wosiek, Phys. Lett. B314 (1993)74. 
[81] P. Bożek, PhD Dissertation. (iANIL Preprint, (iANIL T 92-02.

[82] A. Ludwig. Nucl. Phys. B330 (1990) 639.

[83] A. Bialas and R.C. Hwa, Phys. Lett. B253 (1991) 436.

[84] P. Bożek and M. Płosza jczak, Phys. Lett. B264 (1991) 204.

[85] W. Klein and W. Kinzel, J. Phys. A 14 (1981) L405;

W. Kinzel, Annals of Israel Physical Society 5 (1983) 425.

[86] K. Fialkowski, Acta Phys. Pol. B23 (1992) 1255.

[87] W.Ochs and J.Wosiek, Phys. Lett. B214 (1988) 617.

[88] W. Ochs, Phys. Lett. B247 (1990) 101.

[89] A. Bialas and J. Seixas, Phys. Lett. B250 (1990) 161.

[90] P. Bożek and M. Płoszajczak, Phys. Lett. B251 (1990) 623.

[91] P. Bożek and M. Ploszajczak, Z. Phys. C56 (1992) 473.

[92] L.M. Barbier et al., Phys. Rev. D37 (1988) 1113.

[93] Ph. Brax and R. Peschanski, Int. Jour. Mod. Phys. A7 (1992) 709.

[94] (NA22 Collab.), M. Adamus et al., Phys. Lett. B185 (1987) 200.

[95] P. Lipa, H.C. Eggers, F. Botterweck and M. Charlet, Z. Phys. C54 (1992) 115

[96] A. Bialas and R. Peschanski, Phys. Lett. B207 (1988) 59.

[97] P.Bożek and M. Ploszajczak, Nucl. Phys. A545 (1992) 297c; ibid. Z. Phys. C59 (1993) 585.

[98] U. Frisch and M. Vergassola, Europhys. Lett. 14 (1991) 439.

[99] P. Bożek, Z. Burda , J. Jurkiewicz and M. Ploszajczak, Phys. Lett B265 (1991) 133.

[100] L. Kadanoff, S. Nagel, L. Wu and S. Zhou, Phys. Rev. A39 (1989) 6524.

[101] X. Wu, L. Kadanoff, A. Libchaber and M. Sano, Phys. Rev. Lett. 64 (1990) 2140.

[102] P. Dixon, L. Wu, S. Nagel, B. Williams and J. Carini, Phys. Rev. Lett. 65 (1990) 1108.

[103] M. Jensen, G. Paladin and A. Vulpiani, Phys. Rev Lett. 67 (1991) 208.

[104] G. Paladin and A. Vulpiani, Phys. Rep. 156 (1987) 147.

[105] C. Fong and B. Webber, Nucl. Phys. B355 (1991) 101.

[106] P. Bożek and M. Ploszajczak, Phys. Rev. C44 (1991) 1620.

[107] W. Ochs and J. Wosiek, Phys. Lett. B305 (1993) 144.

[108] G.S. Hawkins, Annu. Rev. Astron. Astrophys. 2 (1964) 149.

[109] E.W. Montroll and R. Simha, J. Chem. Phys. 8 (1940) 721.

[110] R. Shinnar, J. Fluid Mech. 10 (1961) 259.

[111] K. Kang, S. Redner, P. Meakin and F. Leyvraz, Phys. Rev. A33 (1986) 1171.

[112] A.Z. Mekjian, Phys. Rev. Lett. 64 (1990) 2125; Phys. Rev. C41 (1990) 2103.

[113] P. Bożek, M. Ploszajczak and A. Tucholski, Nucl. Phys. A539 (1992) 693.

[114] S.J. Lee and A.Z. Mekjian, Phys. Rev. C47 (1993) 2266.

[115] D.H.E. Gross, A.R. DeAngelis, H.R. Jaqaman, Pan Jicai and R. Heck, Phys. Rev. Lett. 68 (1992) 146;

A.R. DeAngelis, D.H.E. Gross and R. Heck, Nucl. Phys. A537 (1992) 606.

[116] D.H.E. Gross, A. Ecker and A.R. De Angelis, Nucl. Phys. A545 (1992) 187c. 
[117] J. Bjorken, Acta Phys. Pol. B23 (1992) 561;

J.-P. Blaizot and A. Krzywicki, Phys. Rev. D46 (1992) 246;

K. Rajagopal and F. Wilczek, Nucl. Phys. B399 (1993) 395.

[118] H. Heiselberg, C.J. Pethich and D.G. Ravenhall, Phys. Rev. Lett. 61 (1988) 818;

C.J. Pethick and D.G. Ravenhall, Nucl. Phys. A471 (1987) 19c.

[119] L.G. Moretto et al., Phys. Rev. Lett. 69 (1992) 1884;

W. Bauer, G.F. Bertsch and H. Schulz, Phys. Rev. Lett. 69 (1992) 1888;

L.G. Moretto and G.J. Wozniak, LBL Preprint LBL-34176 (1993).

[120] R. Botet and M. Płoszajczak, Phys. Rev. Lett. 69 (1992) 3696;

R. Botet and M. Płoszajczak, Intermittency Patterns of Fluctuations in Disaggregating

Systems, in Complex Fluids, p. 303, ed. L. Garrido, Springer Verlag (Berlin), 1993.

[121] A. Capella, K. Fialkowski and A. Krzywicki, Phys. Lett. B230 (1989) 149;

A. Capella, K. Fiałkowski and A. Krzywicki, in Festschrift L. Van Hove, eds. A. Giovannini and W. Kittel, World Scientific, Singapore, 1990.

[122] A. Bialas, in Festschrift L. Van Hove, eds. A. Giovannini and W. Kittel, World Scientific, Singapore, 1990.

[123] A. Capella, A. Krzywicki and M. Levin, Phys. Rev. D44 (1991) 704.

[124] A. Patel, Phys. Lett. B139 (1984) 394;

L. Van Hove, Z. Phys. C27 (1985) 135.

[125] S.S. Padula and M. Gyulassy, Nucl. Phys. B339 (1990) 378.

[126] M. Lahanas, PhD Dissertation, Frankfurt University (1991), GSI-Report GSI-91-05.

[127] H.T. Elze and I. Sarcevic, Phys. Rev. Lett. 68 (1992) 1988;

R.C. Hwa and M.T. Nazirov, Phys. Rev. Lett. 69 (1992) 741.

[128] H. von Gersdorff, M. Kataja, L. McLerran and P.V. Ruuskanen, Phys. Rev. D34 (1986) 794.

[129] K. Kajantie, R. Raitio and P.V. Ruuskanen, Nucl. Phys. B222 (1983) 152.

[130] G. Baym, B.L. Friman, J.-P. Blaizot, M. Soyeur and W. Czyż, Nucl. Phys. A407 (1983) 541.

[131] H. Kouno, M. Marauyama, K. Saito and F. Takagi, Phys. Rev. D41 (1990) 2903.

[132] A. Hosoya and K. Kajantie, Nucl. Phys. B250 (1985) 666.

[133] G. Baym H. Monien, C.J. Pethick and D.G. Ravenhall, Phys. Rev. Lett. 64 (1990) 1867.

[134] P.J.E. Peebles, The Large Scale Structure of the Universe, Princeton University Press, Princeton (New Jersey), 1980.

[135] W. Bauer, U. Post, D.R. Dean and U. Mosel, Nucl. Phys. A452 (1986) 699;

W. Bauer, G.F. Bertsch and S. Das Gupta, Phys. Rev. Lett. 58 (1987) 863;

J. Desbois, Nucl. Phys. A466 (1987) 724.

[136] D. Stauffer, Introduction to Percolation Theory (Taylor and Francis, London and Philadelphia, Penn., 1985);

R.M. Ziff, Phys. Rev. Lett. 56 (1986) 545.

[137] M. Baldo, A. Causa and A. Rapisarda, Phys. Rev. C48 (1993) 2520.

[138] V. Latora, A. Bonasera and M. Belkacem, LNS Preprint (1993)

[139] R.C. Hwa, Nucl. Phys. B328 (1989) 57.

[140] C.B. Chiu and R.C. Hwa, Phys. Lett. B236 466. 
[141] M. Jędrzejczak, Phys. Lett. B228 (198x) 259.

[142] R.C. Hwa and J.C. Pan, Phys. Rev. D45 (1992) 106.

[143] G. Gustafsson and A. Nilsson, Z. Phys. C52 (1991) 5333.

[144] A. F. Fillipov, Theory Probability : Its Applications 4 (1961) 275;

P.N. Singh and D. Ramkrishna, Comp. and Chem. Eng. 1 (1977) 23;

T.W. Peterson, Aero. Sci. Tech. 5 (1986) 93;

E.D. McGrady and R.M. Ziff, AIChE J. 34 (1988) 2073.

[145] H.H.G. Jellinek and G. White, J. Polymer Sci. 6 (1951) 745;

O. Saito, J. Phys. Soc. Japan 13 (1958) 198;

T.W. Peterson, M.V. Scotto and A.F. Sarofim, Powder Technol., 45 (1985) 87;

R.M. Ziff and E.D. McGrady, J. Phys. A 18 (1985) 3027.

[146] E.D. McGrady and R.M. Ziff, Phys. Rev. Lett. 58 (1987) 892;

Z. Cheng and S. Redner, Phys. Rev. Lett. 60 (1988) 2450.

[147] R. Botet and M. Płoszajczak, Proc. Workshop on Fragmentation Phenomena, Ecole de Physique Theorique, Centre de Physique des Houches, 12 - 17 April 1993, GANIL Preprint P 93/14.

[148] R. Botet and M. Płoszajczak, Proc. XXIII Mazurian Lakes Summer School on Nuclear Physics, Piaski, Poland, 18 - 28 August 1993, GANIL Preprint P 93/31.

[149] E.D. McGrady and R.M. Ziff, Phys. Rev. Lett. 58 (1987) 892.

[150] (ALADIN Collab.), P. Kreutz et al., Nucl. Phys. A556 (1993) 672;

J. Hubele et al., Phys. Rev. C46 (1992) R1577; Z. Physik A340 (1991) 263.

[151] R. Botet and M. Płoszajczak, Phys. Lett. B312 (1993) 30.

[152] M. Gyulassy, in Festschrift L. Van Hove, eds. A. Giovannini and W. Kittel, World Scientific, Singapore, 1990;

P. Carruthers, E.M. Friedlander, C.C. Shih and R.M. Weiner, Phys. Lett. B222 (1989) 487.

[153] A. Bialas, Nucl. Phys. A545 (1992) 285c.

[154] A. Białas, Acta Phys. Pol. B23 (1992) 561.

[155] A. Białas and B. Ziaja, Acta Phys. Pol. B24 (1993) 1509.

[156] P. Grassberger, Nucl. Phys. B120 (1977) 231. 


\section{Figure Captions}

Fig. 1

(a) The integration domain for the bin-averaged second factorial moment in 1D. The point denotes a configuration of two close particles $\left(y_{1} \simeq y_{2}\right)$, which not taken into account in the integration.

(b) The integration domain for the correlation integral. The same two-particle configuration as in 1a is now taken into account in the integration.

\section{Fig. 2}

Examples of the cluster topologies used in the definition of the correlation integral of order 4: (a) the GHP topology, (b) the snake topology and (c) the star topology.

Fig. 3

The experimental results on the dependence of the SFMs of the fragment charge distribution on the bin size for $\mathrm{Au}$ - emulsion interaction at $E / A \simeq 1 \mathrm{GeV}$ (from ref. [35]). The events are selected according to the total fragment multiplicity (left-hand side) or to the number of heavy fragments with the charge $Z \geq 3$ (right-hand side). Solid lines represent linear fits to the experimental data.

\section{Fig. 4}

The dependence of the second SFM on the total number of cells in the 1D, 2D and 3D analysis of the $e^{+} e^{-}$annihilation by the DELPHI Collaboration [56].

Fig. 5

The dependence of the second SFM on the total number of cells in the $1 \mathrm{D}, 2 \mathrm{D}$ and $3 \mathrm{D}$ analysis of the $\pi / \mathrm{K}-\mathrm{p}$ collisions by the NA22 Collaboration (from [48]).

Fig. 6

The dependence of the second correlation integral on $Q^{2}$ in the NA22 data (full circles) and in the FRITIOF2.0 (open circles), FRITIOF2.0 with Dalitz decays (triangles) and FRITIOF2.0 with Dalitz decay and $\gamma$-conversion (squares), for all charged particles (left-hand side), negative charge particles (middle) and unlike-charge particles (righthand side) (from [48]).

\section{Fig. 7}

Density strip integrals $C_{q}(q=2-5)$ in 3D analysis for all-charged (open circles) and same charged (black circles) combinations in $p-\bar{p}$ collisions at $630 \mathrm{GeV}$ by the UA1 Collaboration (from [63]).

\section{Fig. 8}

The dependence of the second correlation integral on the number of cells in the $3 \mathrm{D}$ analysis of the NA35 Collaboration for : (a) $p-A u$, (b) $O-A u$ and (c) $S-S$ data (from [69]). 


\section{Fig. 9}

An example of the random cascading in the $\alpha$-model [3] for the branching number $\lambda=2$.

\section{Fig. 10}

The dependence of the second SFM $F_{2}$ on the size of the rapidity bin $\delta y$, as obtained from eq. (3.16) with $n=2$ for three different values of the correlation range $R_{\text {corr }}$. The dashed-dotted line represents the results obtained using the non-singular, short range correlation ansatz for the reduced density (2.47) with the parameters $\gamma=0.25$ and $\xi=1.1$. All the curves are normalized at $\delta y=1$. (From ref. [90] .)

\section{Fig. 11}

(a) The SFM $F_{2}$ as a function of $\ln M$ for the modified $\alpha$-model with the branching number $\lambda=2$ and the probability distribution of random factors $P(w)=0.5 \delta(w-$ $1.2)+0.5 \delta(w-0.8)$ (the weak intermittency limit). The solid triangles, the open triangles, the solid boxes and the open boxes correspond to the cut-off values $\epsilon=3 \star 10^{-3}$, $1 \star 10^{-3}, 5 \star 10^{-4}$ and $2 \star 10^{-4}$ respectively. The solid line denotes the results of the standard $\alpha$-model $(\epsilon=0)$.

(b) The rescaled SFM $G_{2}$ as a function of $\Theta=\ln M / \ln (1 / \epsilon)$, for the same parameters as in (a). The results for different values of the cut-off $c$ are denoted by the same symbols as in (a). (From ref. [97] .)

\section{Fig.12}

The dependence of scaled factorial moments $F_{i} \quad(i=2,3)$, on the bin size for different values of the tuning parameter $x$ in the Mekjian model [112] with $A=216$ constituents. $x<<1$ correspond to fusion or evaporation modes. For $x=1$ one obtains the scale-invariant power-law for fragment distribution with the critical exponent $\tau=1$ whereas $x>>1$ corresponds to the dissociation of the system. (From ref. [113].)

\section{Fig.13}

The dependence of scaled factorial moment $F_{i} \quad(i=2,3)$ calculated for the ensemble of events in the range of the tuning parameters $x$ from $x_{\min }=0.5$ to $x_{\max }=3.5$ is plotted as a function of the size of the bin in the Mekjian model with $A=216$ constituents. The solid line denotes $F_{2}$ whereas $F_{3}$ is depicted with the dashed line. (From ref. [113] .)

\section{Fig.14}

The space-time evolution of the system in the nuclear collision. The maximal range $\tau_{\text {corr }}$ of the correlations, which are build between the two proper times $\tau_{1}$ and $\tau_{2}$ is shown. The variable $|x|$ corresponds to $|\vec{r}|$ in $(3+1)$-dimensions.

\section{Fig.15}

The dependence of the second SFM $F_{2}$ on the size of the rapidity bin $\delta y$, as obtained from eq. (4.9) for three different freeze out times $\tau_{2}=4 \mathrm{fm}$ (the solid line), $6 \mathrm{fm}$ (the dotted line) and $10 \mathrm{fm}$ (the dashed line). The parameters are set to $\tau_{1}=3 \mathrm{fm} / \mathrm{c}$, 
$q=0.25$ and $\sigma=0.3$. All the curves are normalized at $\delta y=1$. (From ref. [90] .)

\section{Fig.16}

The dependence of the second SFM $F_{2}$ on the size of the rapidity bin $\delta y$, as obtained from eq. (4.9) for three different freeze out times $\tau_{2}=4 \mathrm{fm}$ (the solid line), $6 \mathrm{fm}$ (the dotted line), $10 \mathrm{fm}$ (the dashed line) and $20 \mathrm{fm}$ (the dash-dotted line). The parameters are set to $\tau_{1}=3 \mathrm{fm} / \mathrm{c}, q=0.5$ and $\sigma=0.5$. All the curves are normalized at $\delta y=1$. (From ref. [90] .)

\section{Fig. 17}

The dependence of the second SFM $F_{2}$ on the size of the rapidity bin $\delta y$, as obtained from eq. (4.9) for $O-A u$ collisions using $\tau_{2}=24 \mathrm{fm} / \mathrm{c}, R_{t u}=7.2 \mathrm{fm} / \mathrm{c}$ (the dashed line) and for $S-S$ collisions using $\tau_{2}=5.5 \mathrm{fm} / \mathrm{c}, R_{t u}=5.5 \mathrm{fm}$ (the solid line). The parameters are set to $\tau_{1}=2 \mathrm{fm} / \mathrm{c}, q=0.5$ and $\sigma=0.5$. All the curves are normalized at $\delta y=1$.

\section{Fig.18}

The same as in Fig. 17 but for $q=1$ and $\sigma=0.8$.

\section{Fig.19}

(a) Energy density at three different evolution times for the gaussian random hadronization sources with the width $s=0.2$.

(b) Energy density at three different evolution times for the fractal random hadronization sources. (From ref. [106] .)

\section{Fig. 20}

(a) The dependence on the size of the rapidity bin $\delta y$ for the moments $F_{2}$ (the solid line), $F_{3}$ (the dotted line) and $F_{4}$ (the dashed line) of the distributions shown in Fig. 19 a .

(b) The dependence on the size of the rapidity bin $\delta y$ for the moments $F_{2}$ (the solid line), $F_{3}$ (the dotted line) and $F_{4}$ (the dashed line) of the distributions shown in Fig. 19b. (From ref. [106] .)

\section{Fig. 21}

The dependence of scaled factorial moments $F_{2}$ (the dashed line), $F_{3}$ (the solid line ) on the bin size in the $1 \mathrm{D}$ bond percolation model for different values of the bond activation probabilities $q[113]$. The value of the scale $\ln \delta_{j u m p} s$ at which the attractive correlations prevail is estimated according to eq. (4.25) and is denoted by the arrow.

\section{Fig. 22}

The dependence of scaled factorial moments $F_{q}$ for $q=2-5$ on the bin size in the $3 \mathrm{D}$ bond percolation model with $6^{3}$ sites, for different values of the bond activation probabilities $q[35]$.

\section{Fig. 23}

The dependence of scaled factorial moments $F_{q}(q=3,4,5)$ on the value of $F_{2}$ in 
the experimental data of ref. [4:3] and in the 31) bond percolation model with $6^{3}$ sites and random bond activation probabilities. The events are selected according to the number of intermediate mass fragments of size $s_{f_{r}} \geq 5$ in the calculation and of charge $Z \geq 3$ in the data. (From ref. [44].)

\section{Fig. 24}

The inclusive mass distribution and the corresponding SFMs for $q=2-5$ plotted as a function of the bin size for events with initial temperatures $T=4,5$ and $10 \mathrm{MeV}$. The calculations are performed using the classical molecular dynamics [138] .

Fig. 25

Schematic representation of a topological sequence of the binary sequential fragmentation of the cluster labelled $N$ in the fragmentation-inactivation binary model [148] .

Fig. 26

The scaled factorial moments $F_{2}(M)$ of the fragment size distribution are shown for selected exponents $\alpha$ and $\beta$ at the transition line of the fragmentation inactivation binary model [120] $(N=256): \alpha=-1 / 2, \beta=0, I_{1}=2 \sqrt{\pi} \Gamma(27 / 20) / \Gamma(37 / 20)$ (open points) , $\alpha=0, \beta=1, I_{1}=3 / 37$ (open squares), $\alpha=1, \beta=3, I_{1}=137 / 8778$ (black points) and $\alpha=2, \beta=5, I_{1}=28709 / 8738730$ (black squares). For all these cases $\tau=1.85$. The case $\alpha=0, \beta=0, I_{1}=1$ in the shattered phase is shown for comparison. (From ref. [148].)

Fig. 27

The two-particle correlation function for like-sign particles from the $p$ - $\bar{p}$ collisions at $630 \mathrm{GeV}[63]$.

Fig. 28

The two-particle distribution function $D_{2}(k)$, as obtained from eq. (4.65), for different strength of the intermittency correlations $\nu_{2}=1.0$ (the solid line), $\nu_{2}=0.5$ (the dotted line) and for the uncorrelated sources (the dashed line). (From ref. [106] .)

Fig. 29

The two-particle distribution function $D_{2}(k)$ (eq. (4.65)) for the emission from a surface is shown for different strength of the intermittency correlations $\nu_{2}=1.0$ (the solid line), $\nu_{2}=0.5$ (the dotted line) and for the uncorrelated sources (the dashed line). (From ref. [106] .) 
Table 1.

\begin{tabular}{|c|c|c|c|c|}
\hline Moment & Reaction & Data [67] & Data (rescaled multiplicity) & Model \\
\hline \hline $\mathrm{F}_{2}$ & $\mathrm{p}-\mathrm{Em}$ & 0.019 & 0.019 & 0.016 \\
$\mathrm{~F}_{2}$ & O-Em & 0.016 & 0.003 & 0.006 \\
$\mathrm{~F}_{3}$ & O-Em & 0.042 & 0.010 & $\ldots$ \\
$\mathrm{F}_{2}$ & $\mathrm{~S}-\mathrm{Em}$ & 0.012 & 0.0015 & 0.003 \\
$\mathrm{~F}_{3}$ & $\mathrm{~S}-\mathrm{Em}$ & 0.028 & 0.006 & $\ldots$ \\
\hline
\end{tabular}

Slopes of the SFMs $F_{2}$ and $F_{3}$ for different reactions are compared to the rescaled multiplicity data for $p-E m$ reaction (eq. 3.1). The estimate of the slope from the theoretical model presented in this chapter is given for $F_{2}$. 


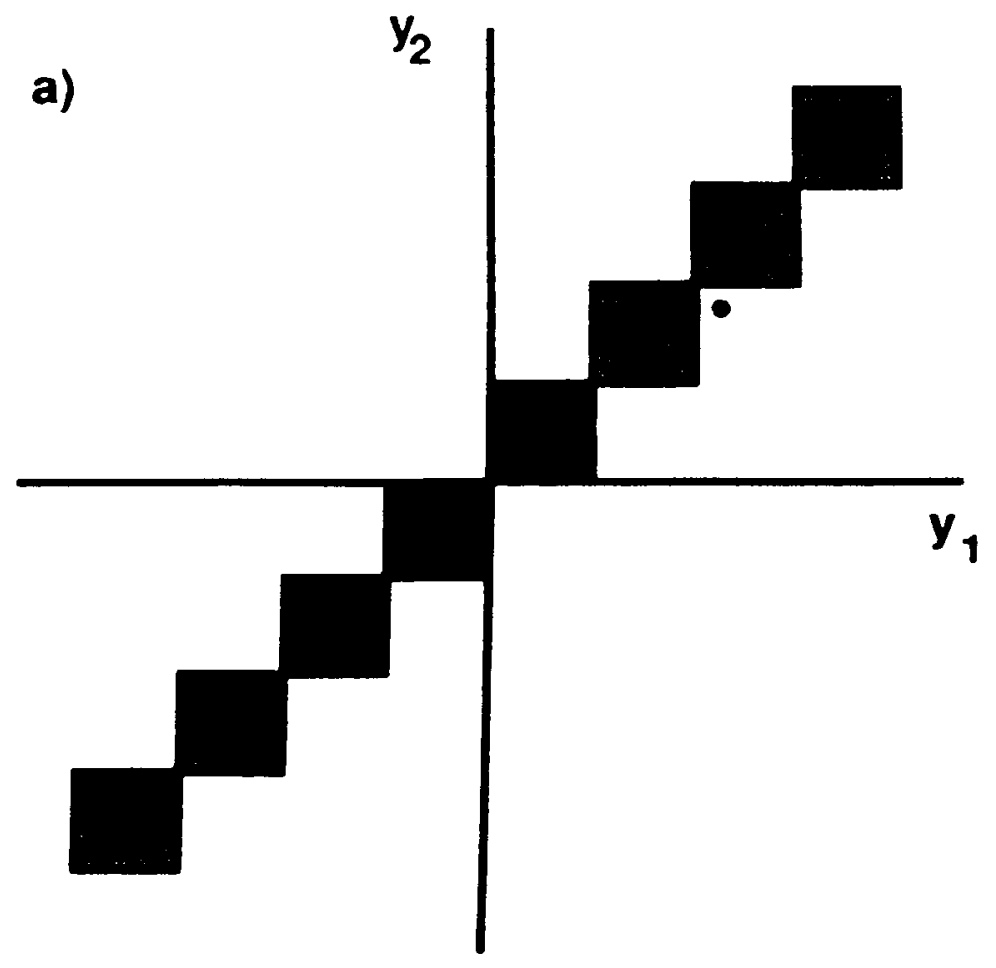

Fig. 1a 


$$
y
$$




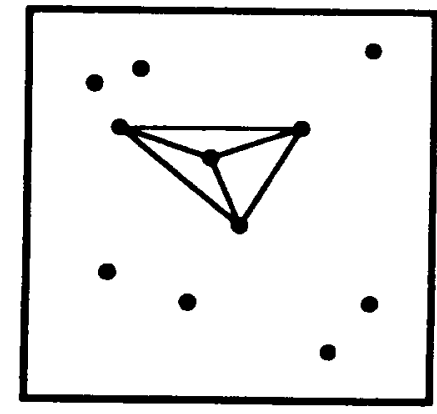

a)

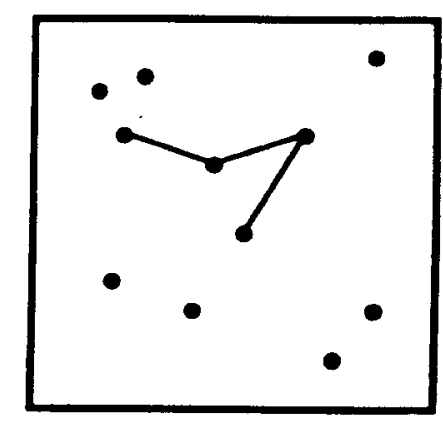

b)

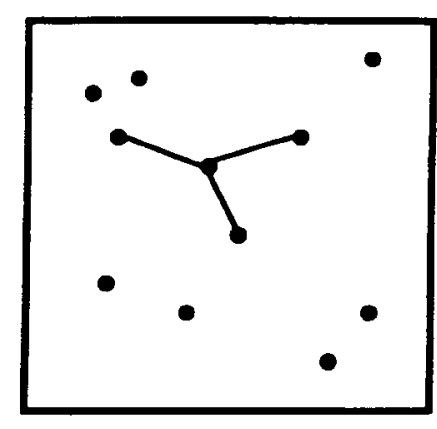

c)

Fig. 2 


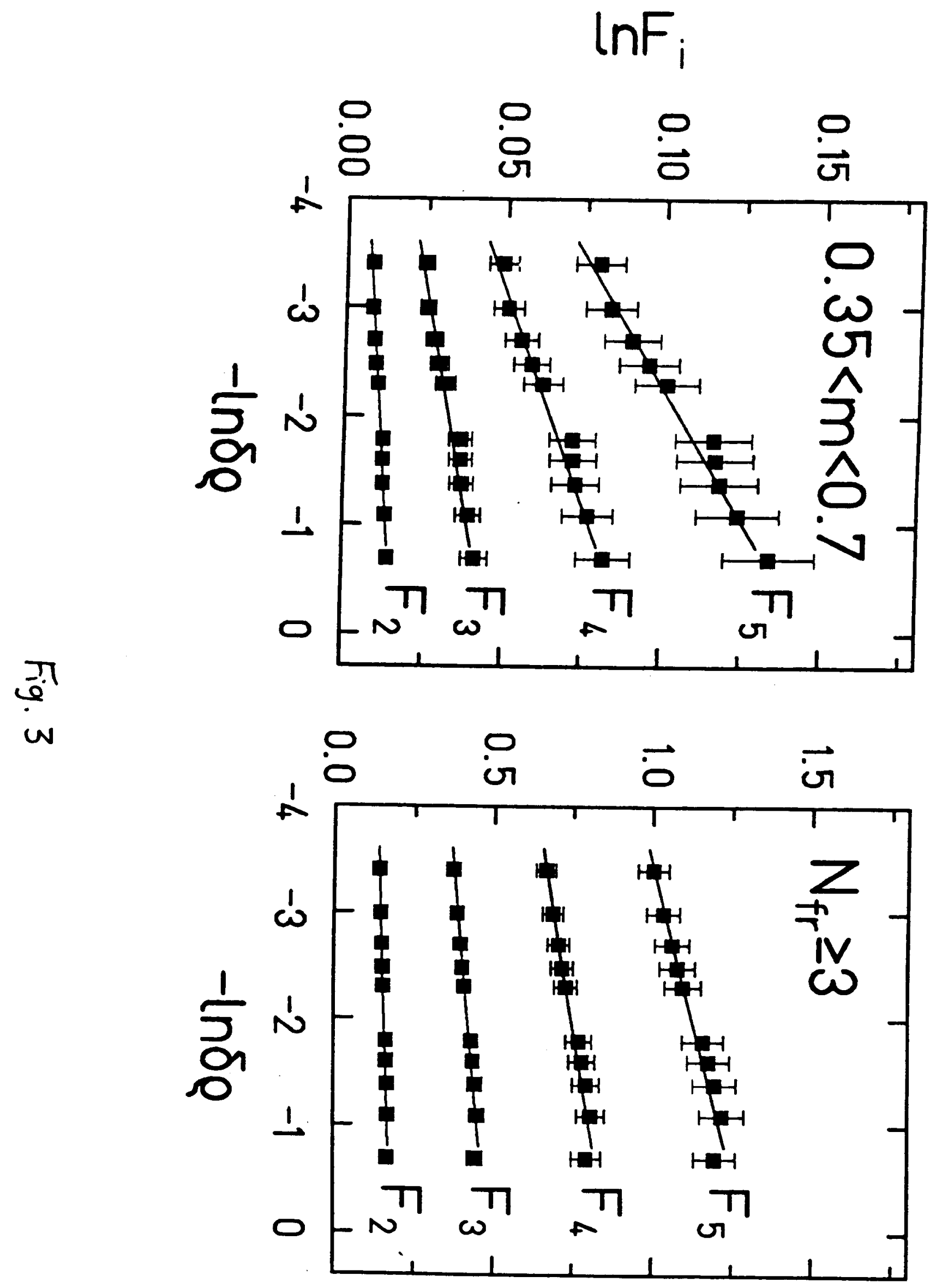




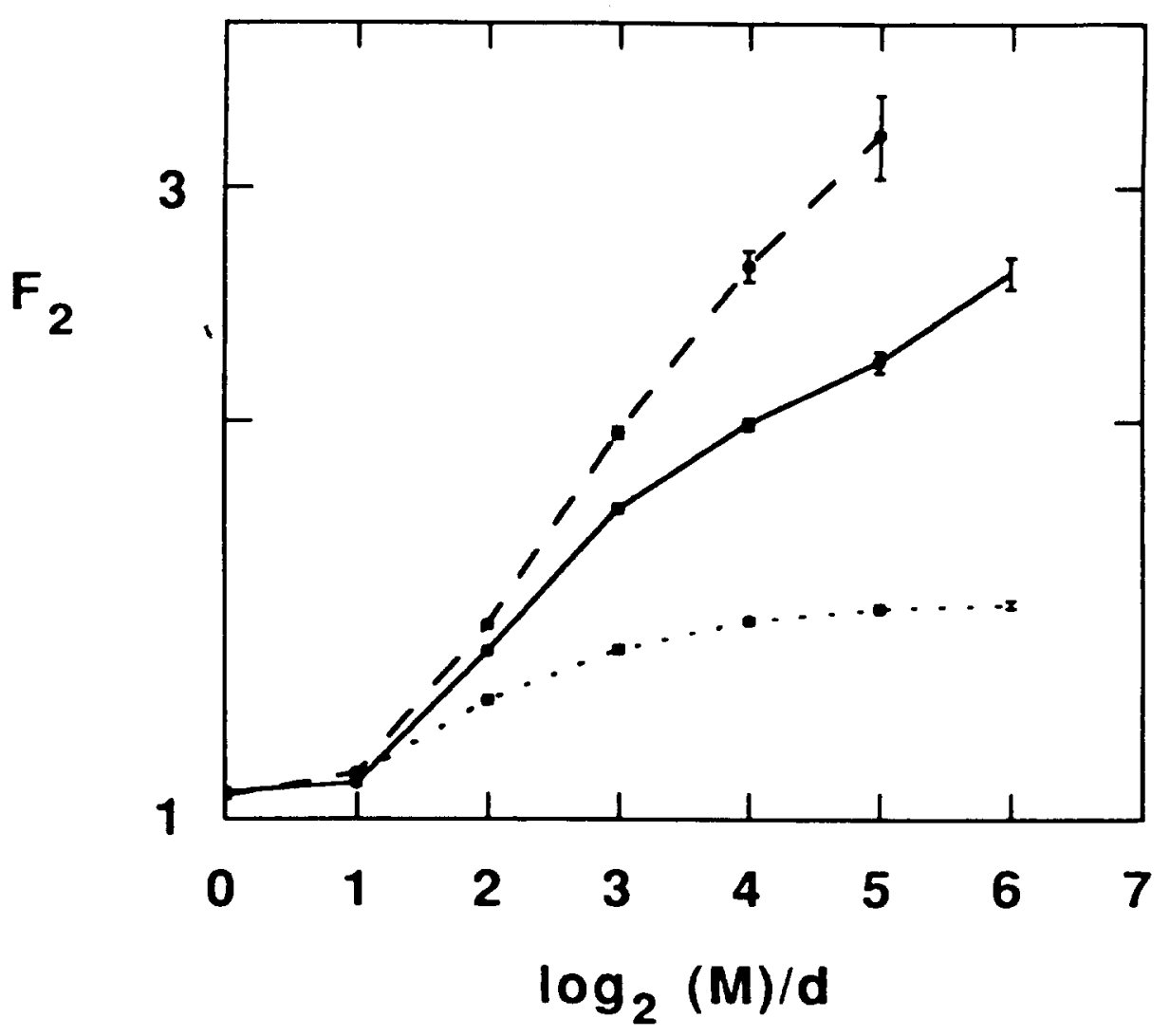

Fig. 4 


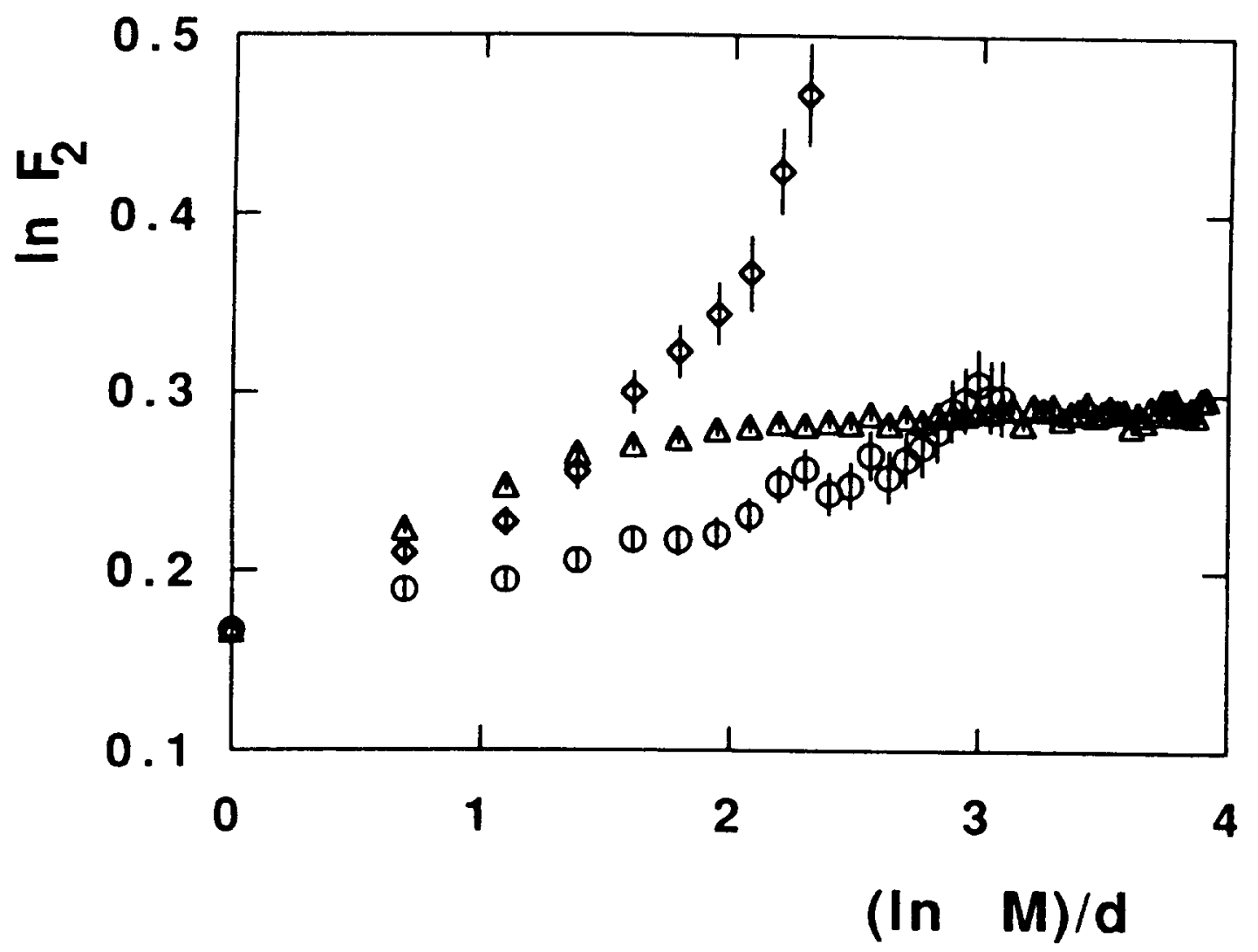

Fig. 5 


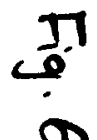

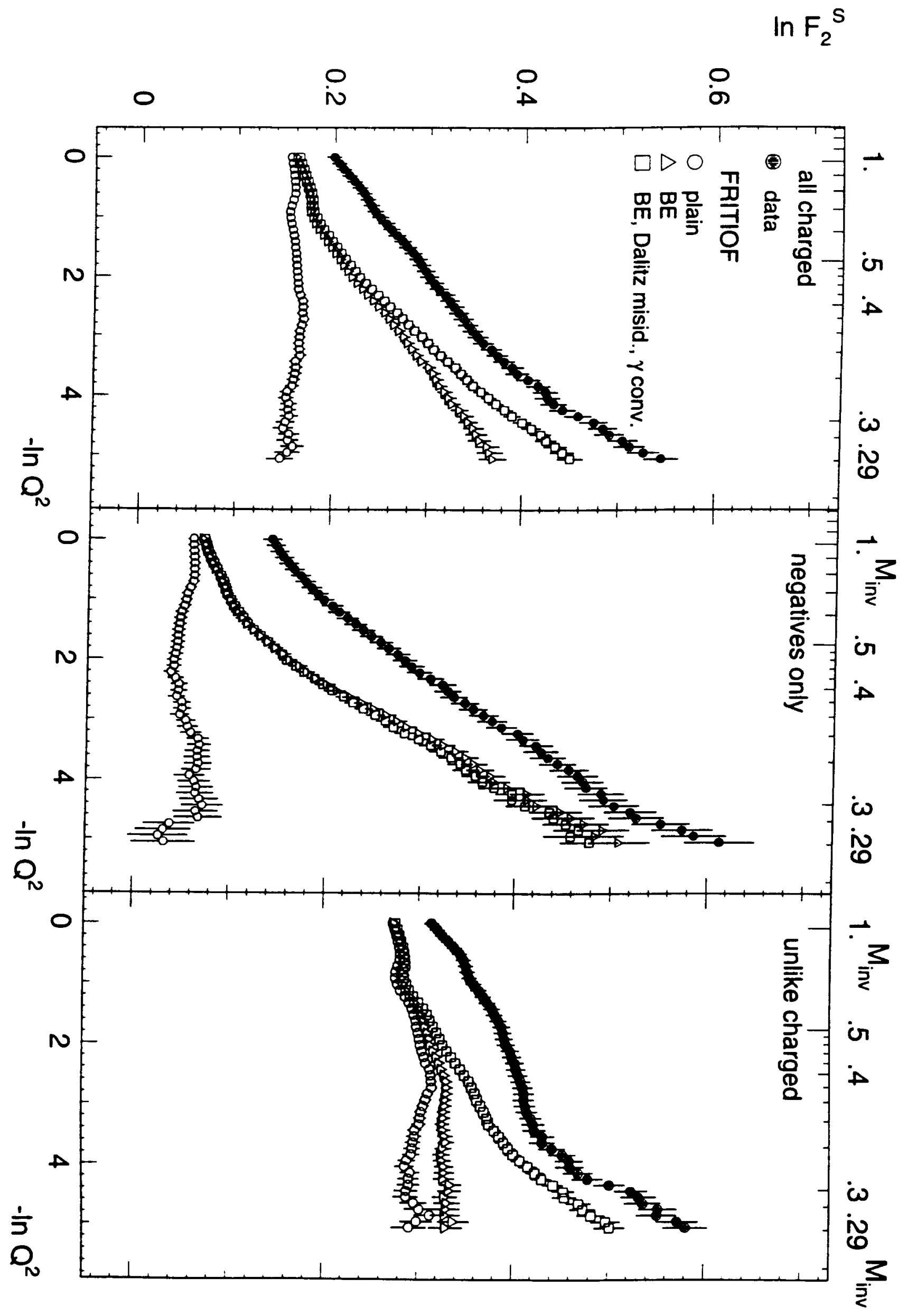




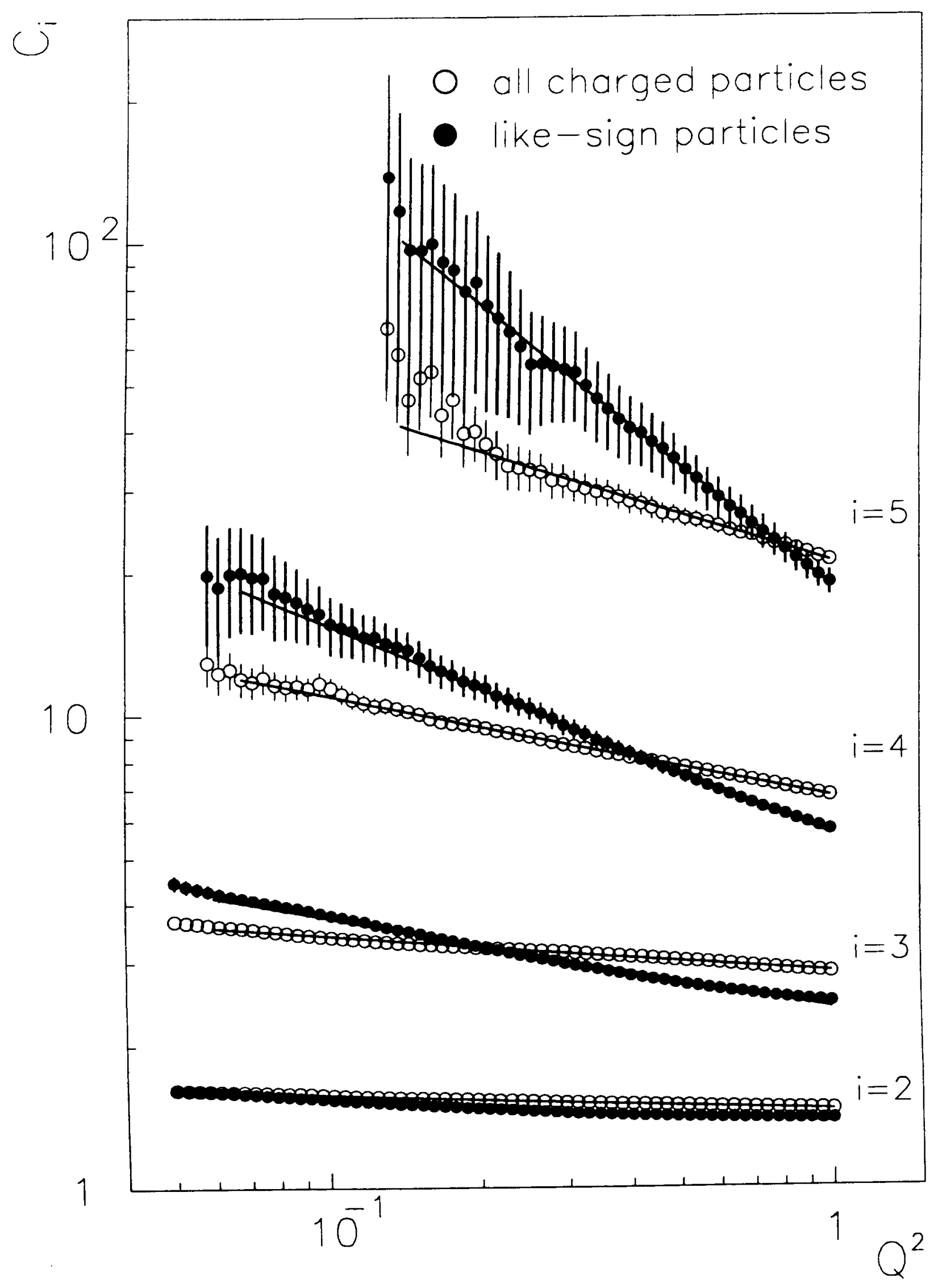

Fia. 7 


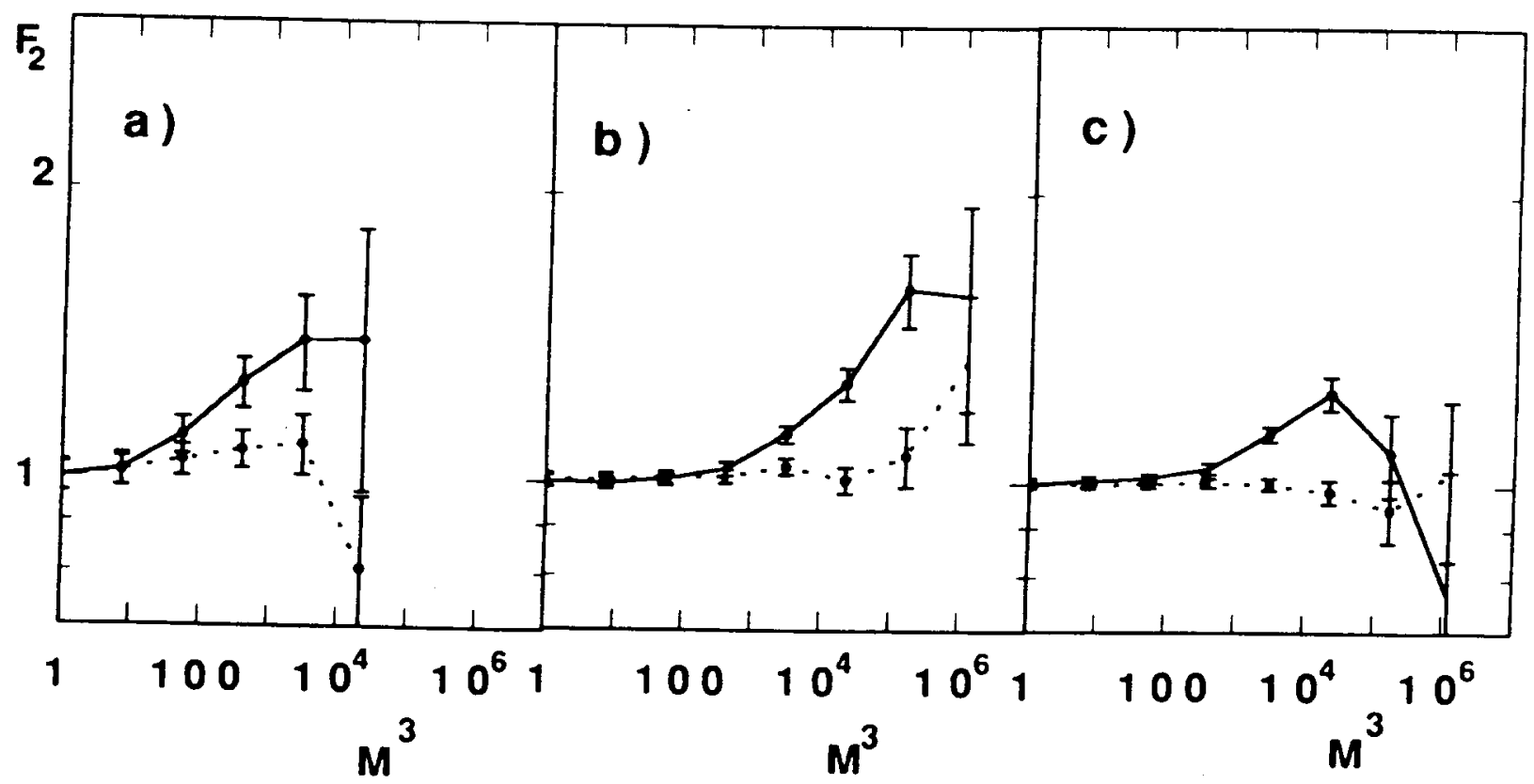

Fig. 8 


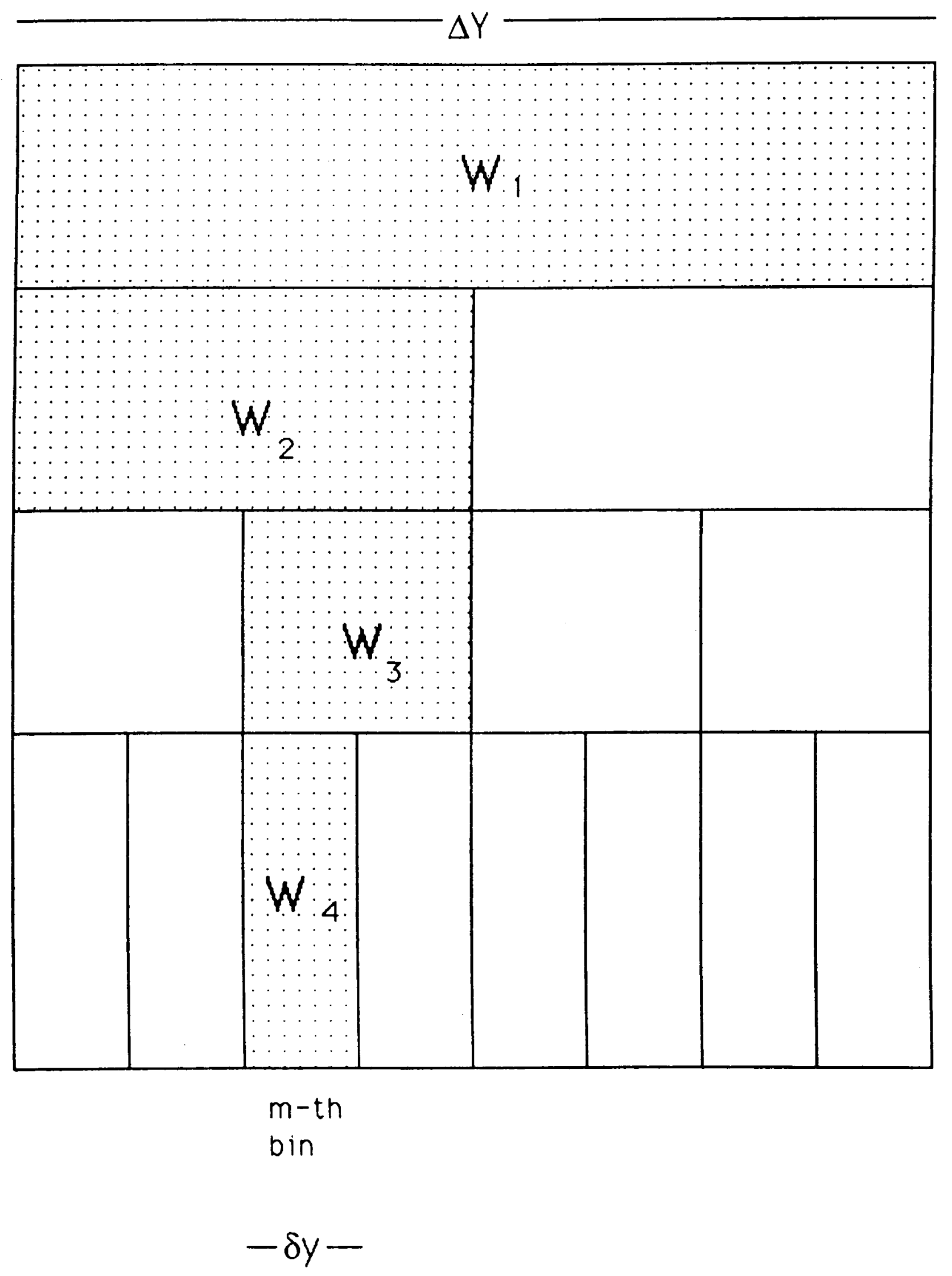

Fig. 9 


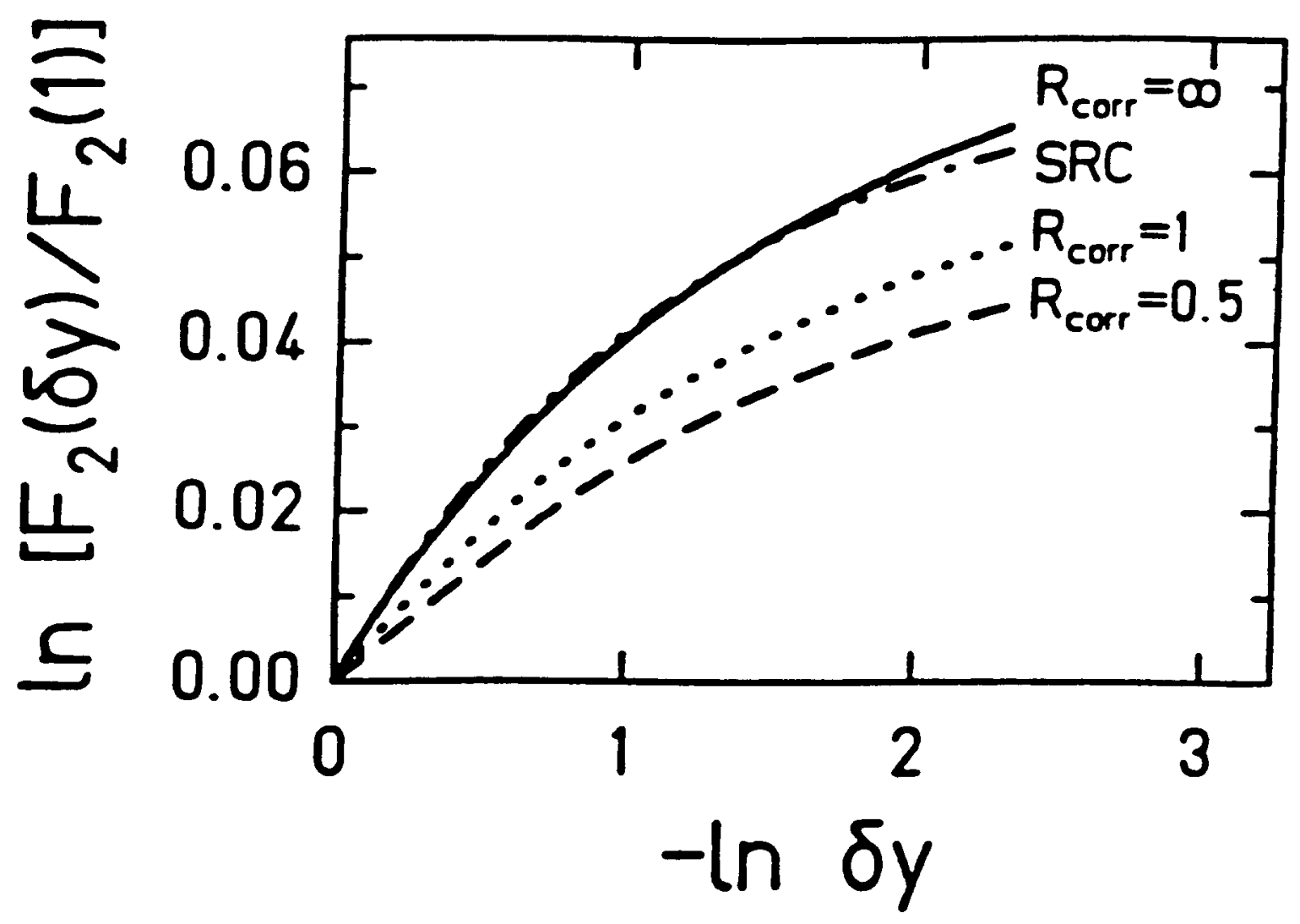

Fig. 10 
$\overrightarrow{0}$
$\overrightarrow{0}$
$\overrightarrow{0}$

$\ln \mathrm{F}_{2}(\mathrm{M})$

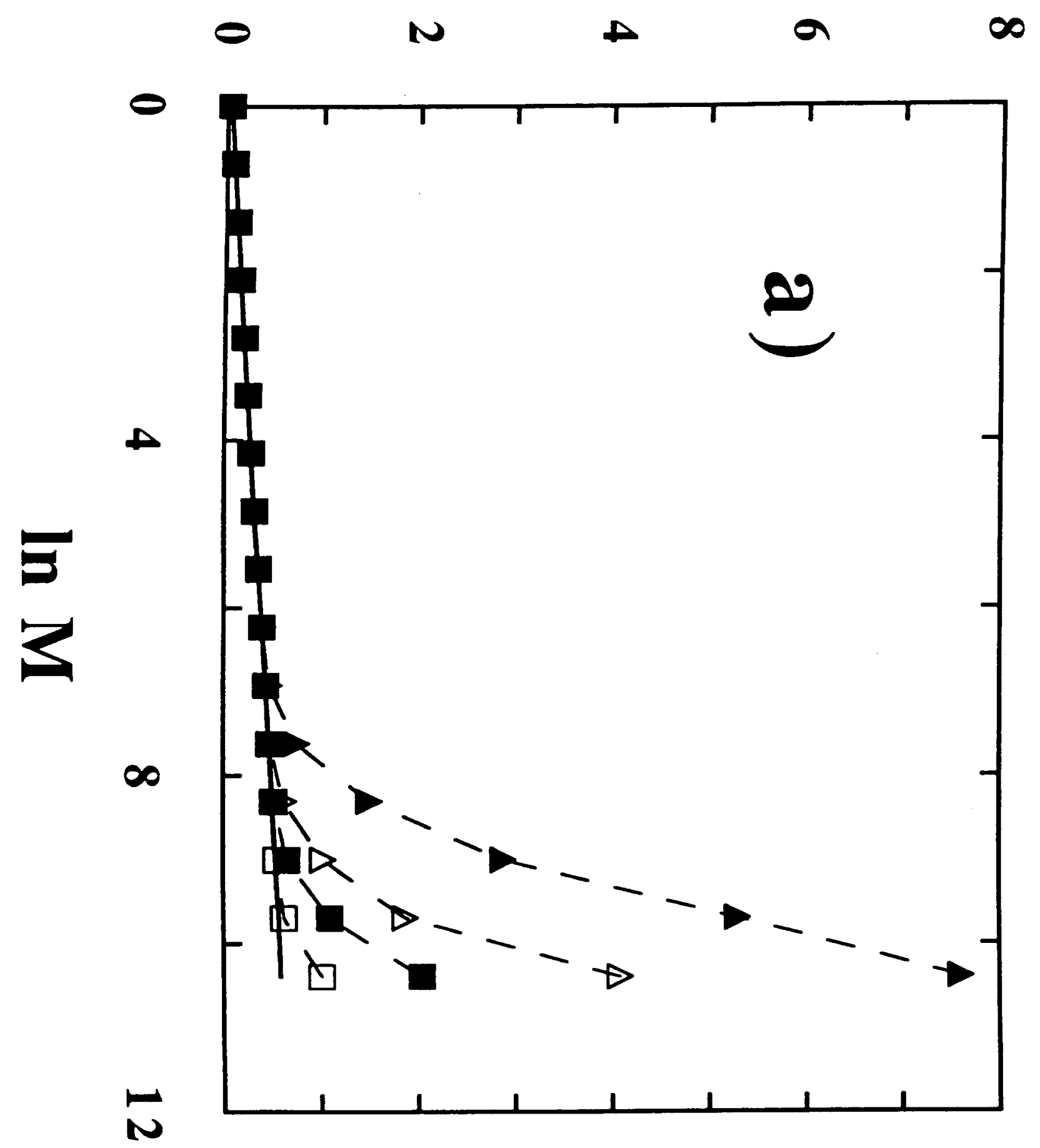


章

$\mathbf{G}_{2}(\Theta)$
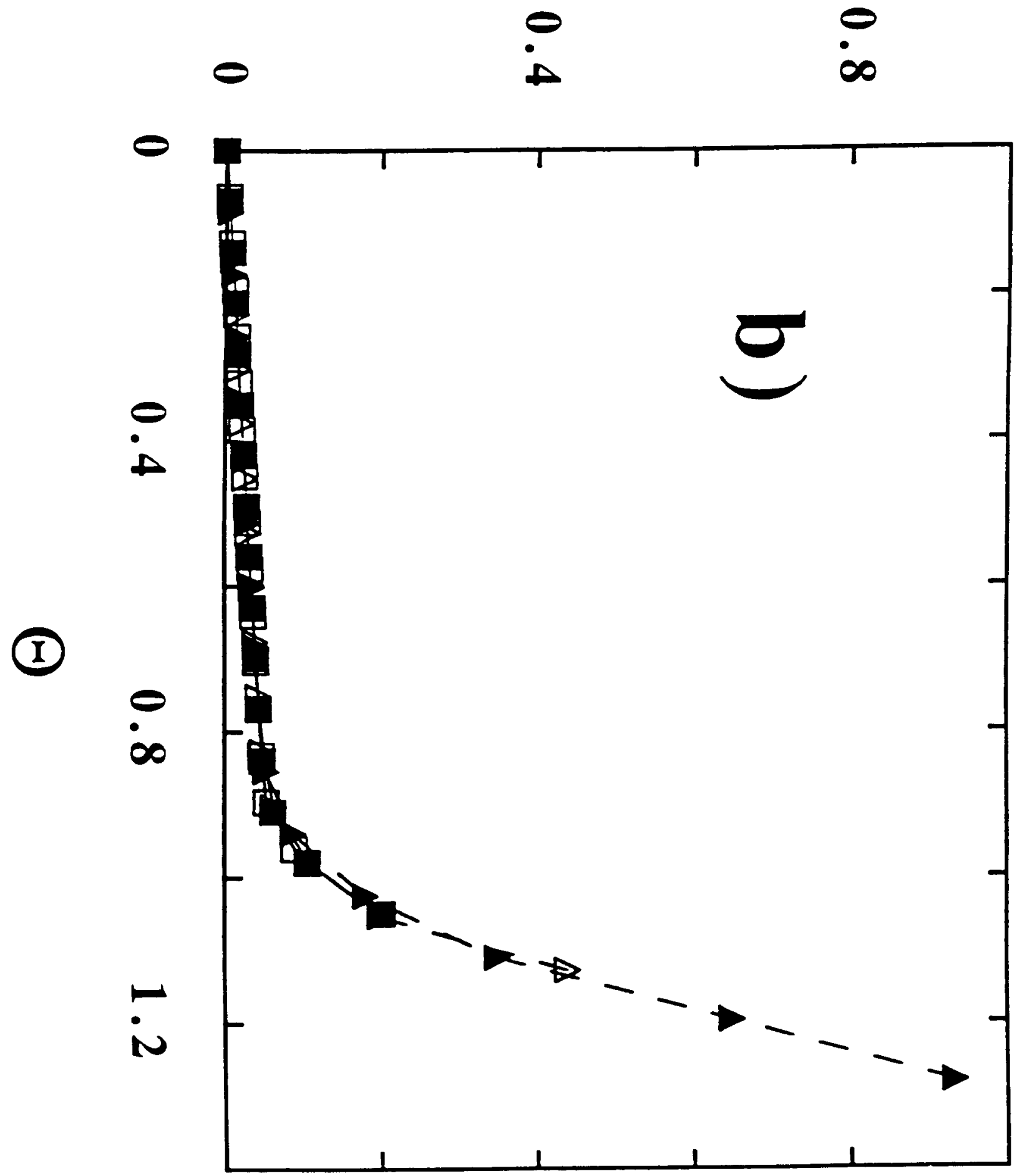

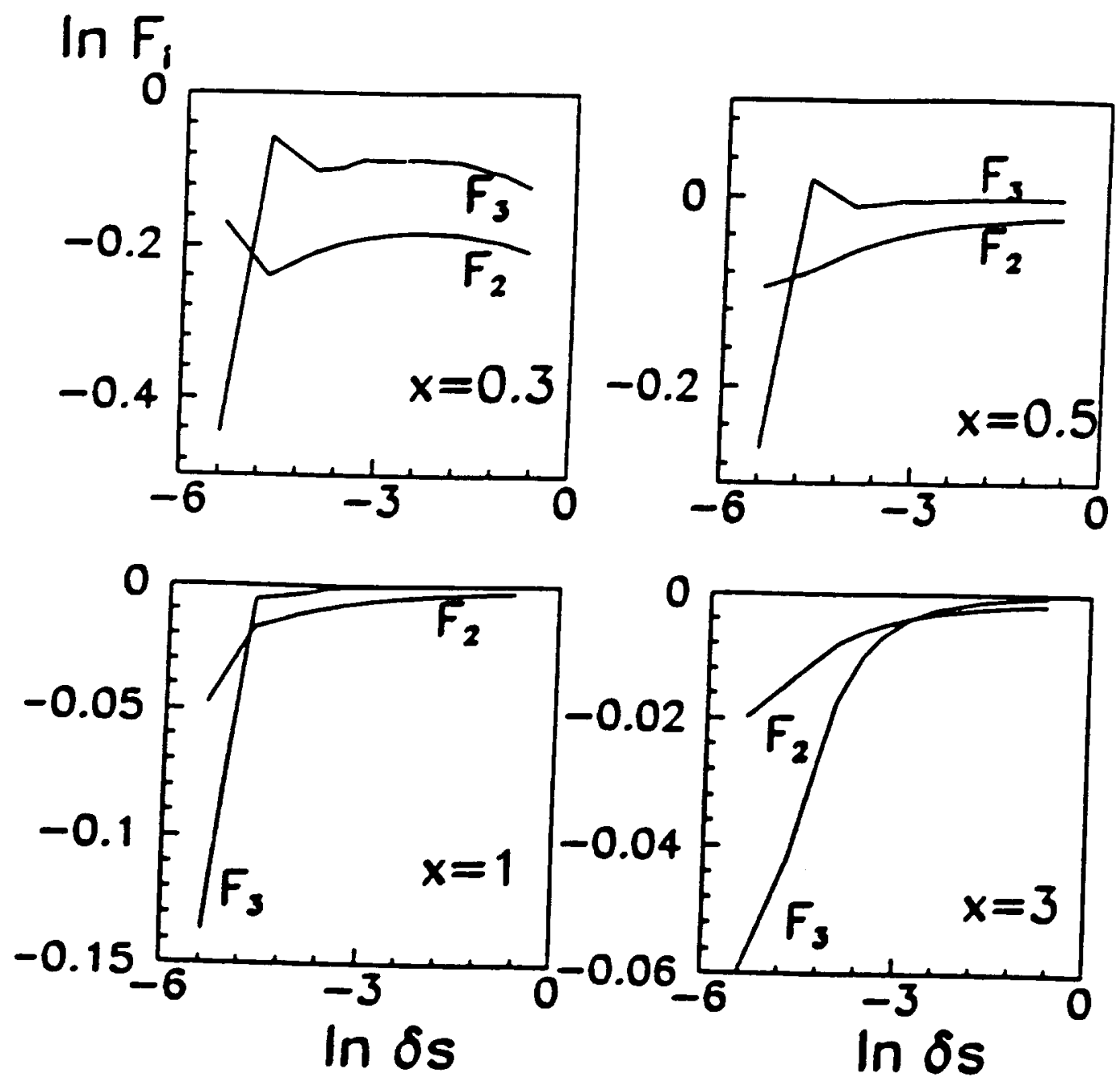

Fig. 12 


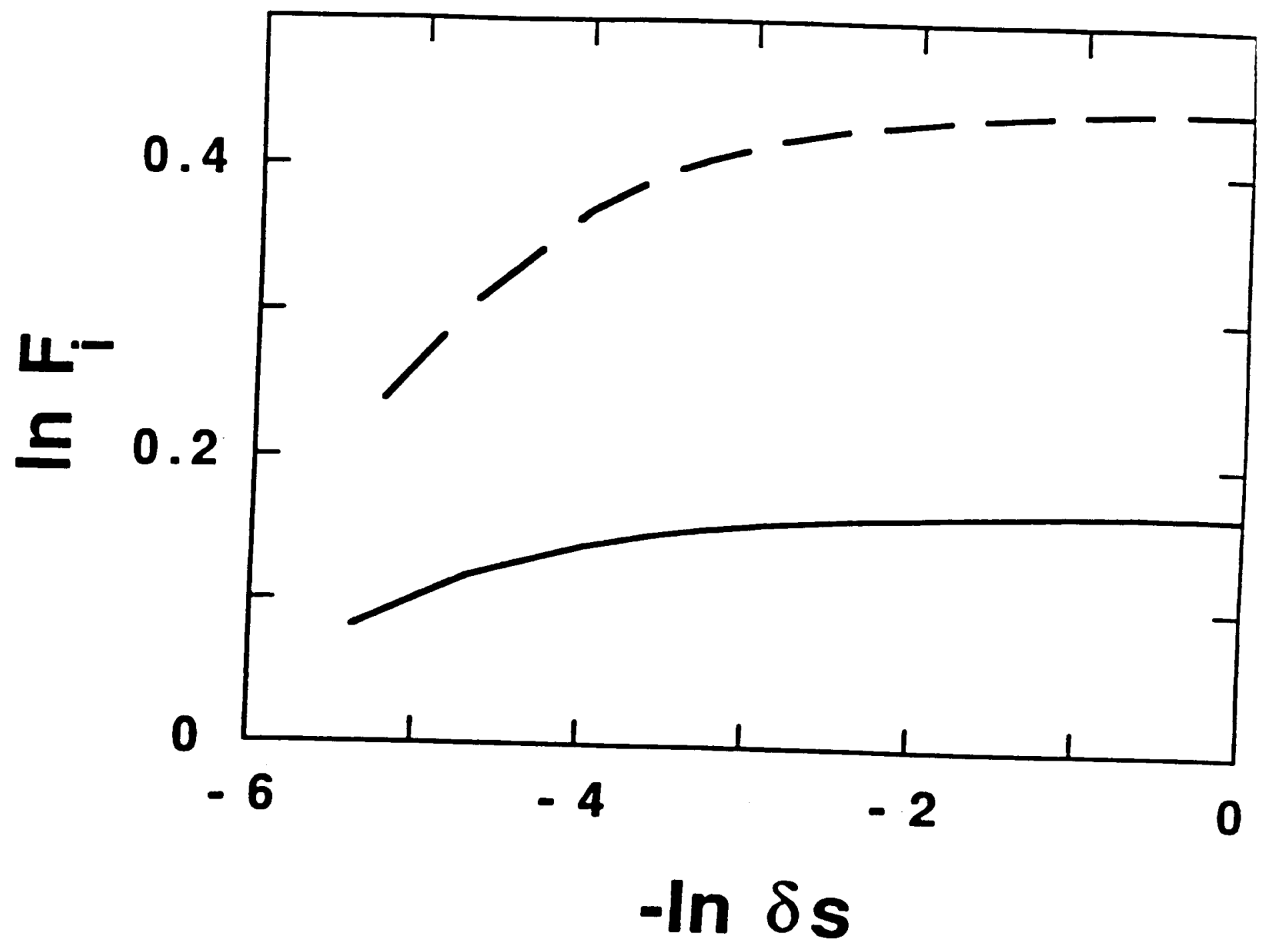

Fig. 13 


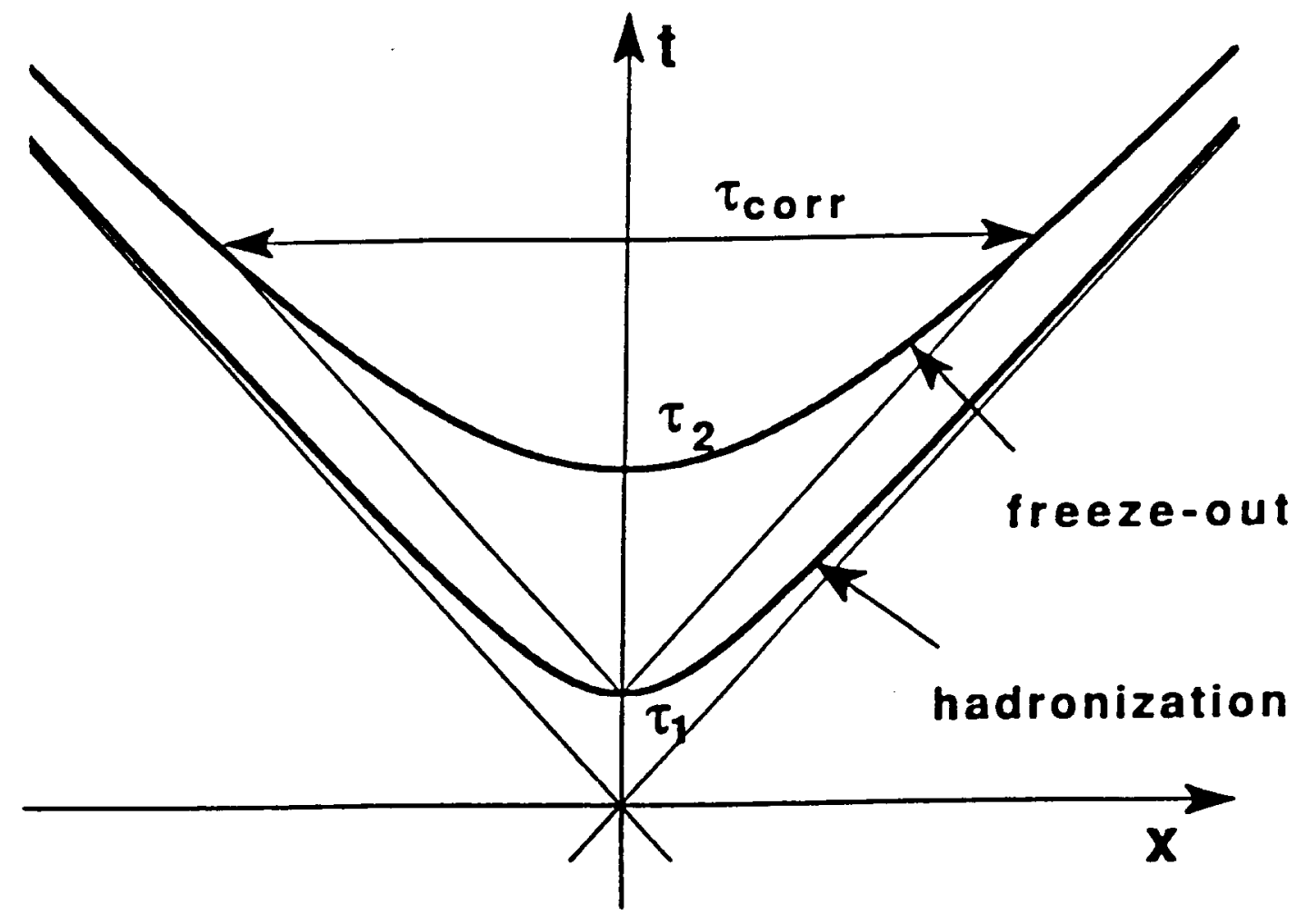

Fig. 14 


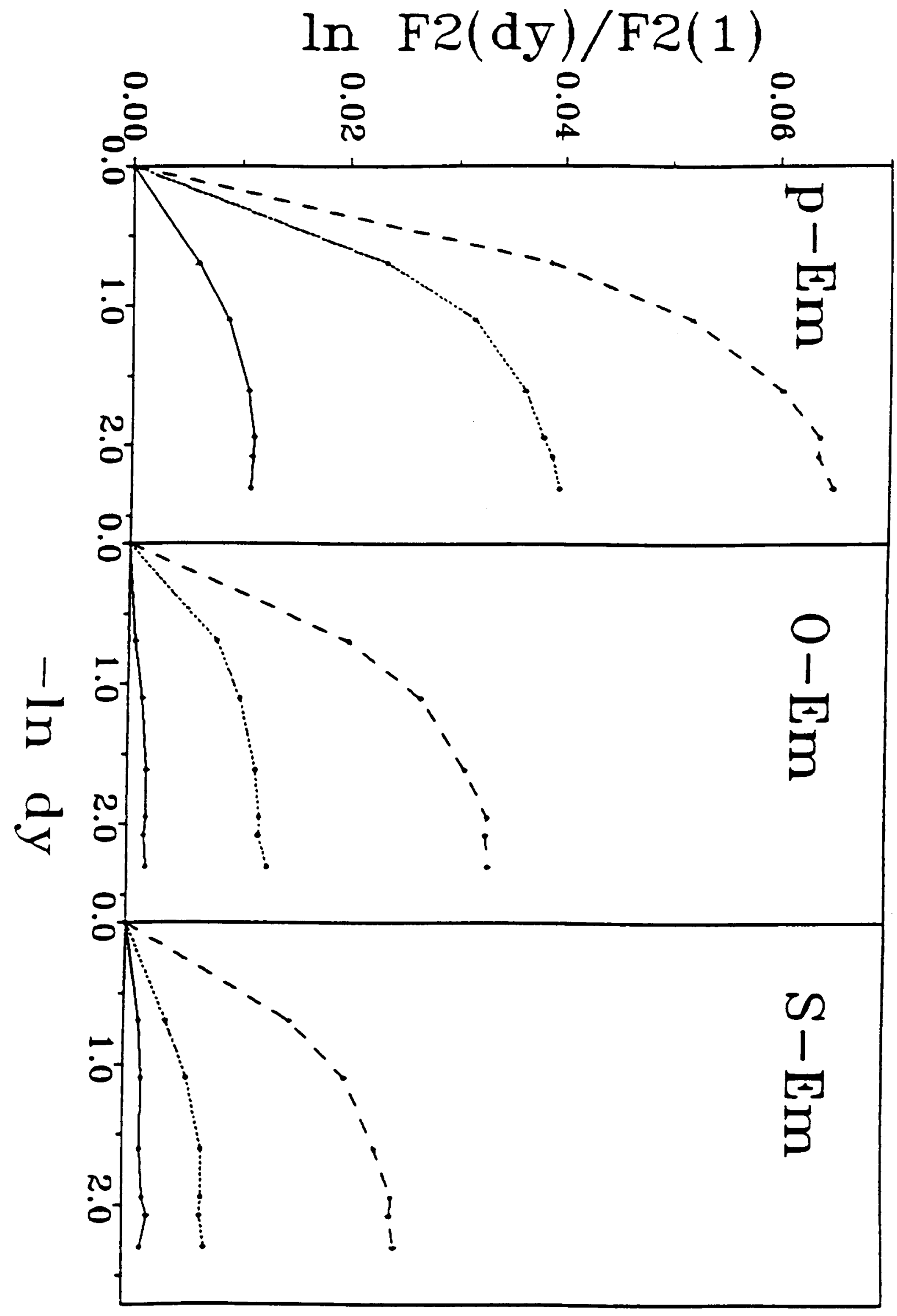

요

जे 
T.
$\stackrel{n}{n}$

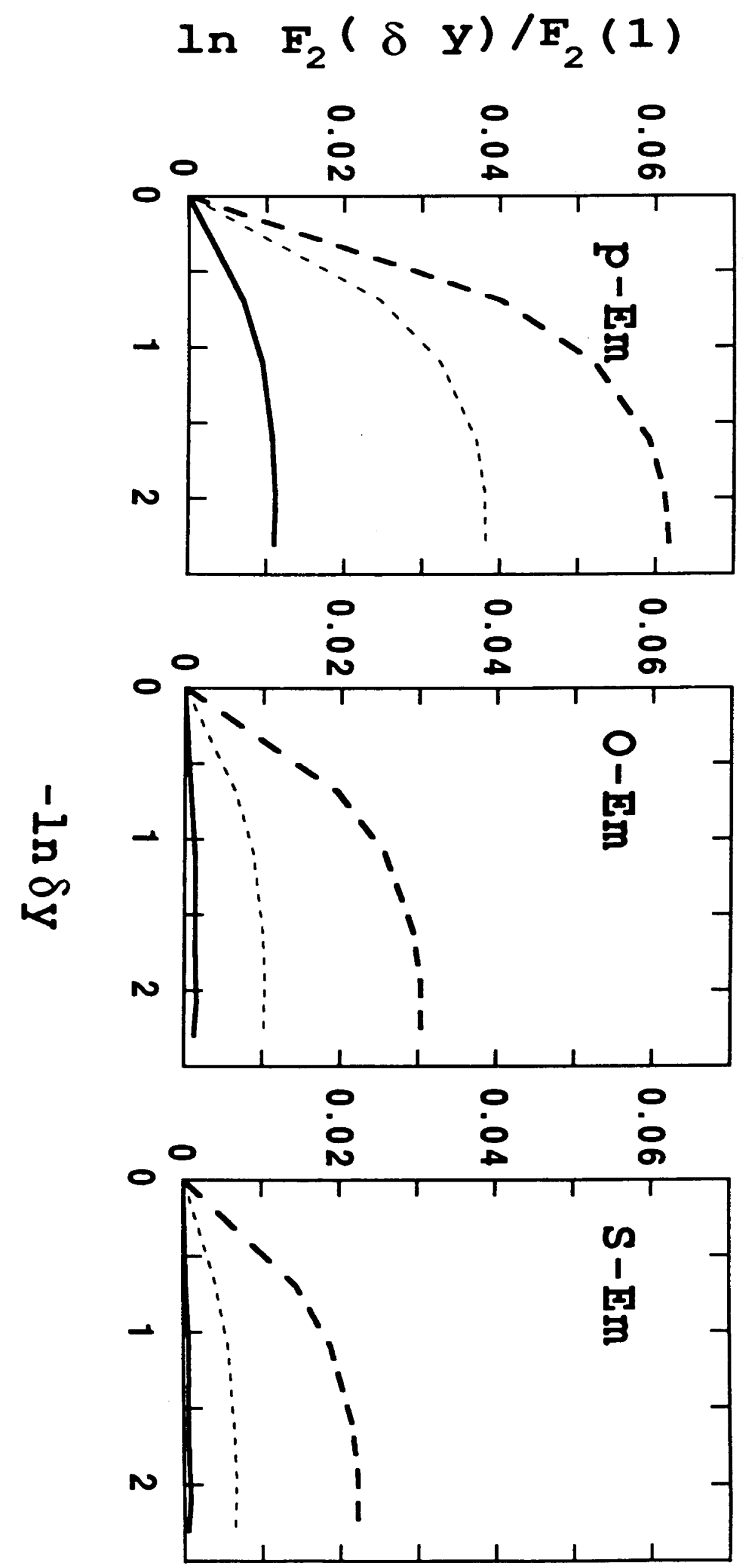




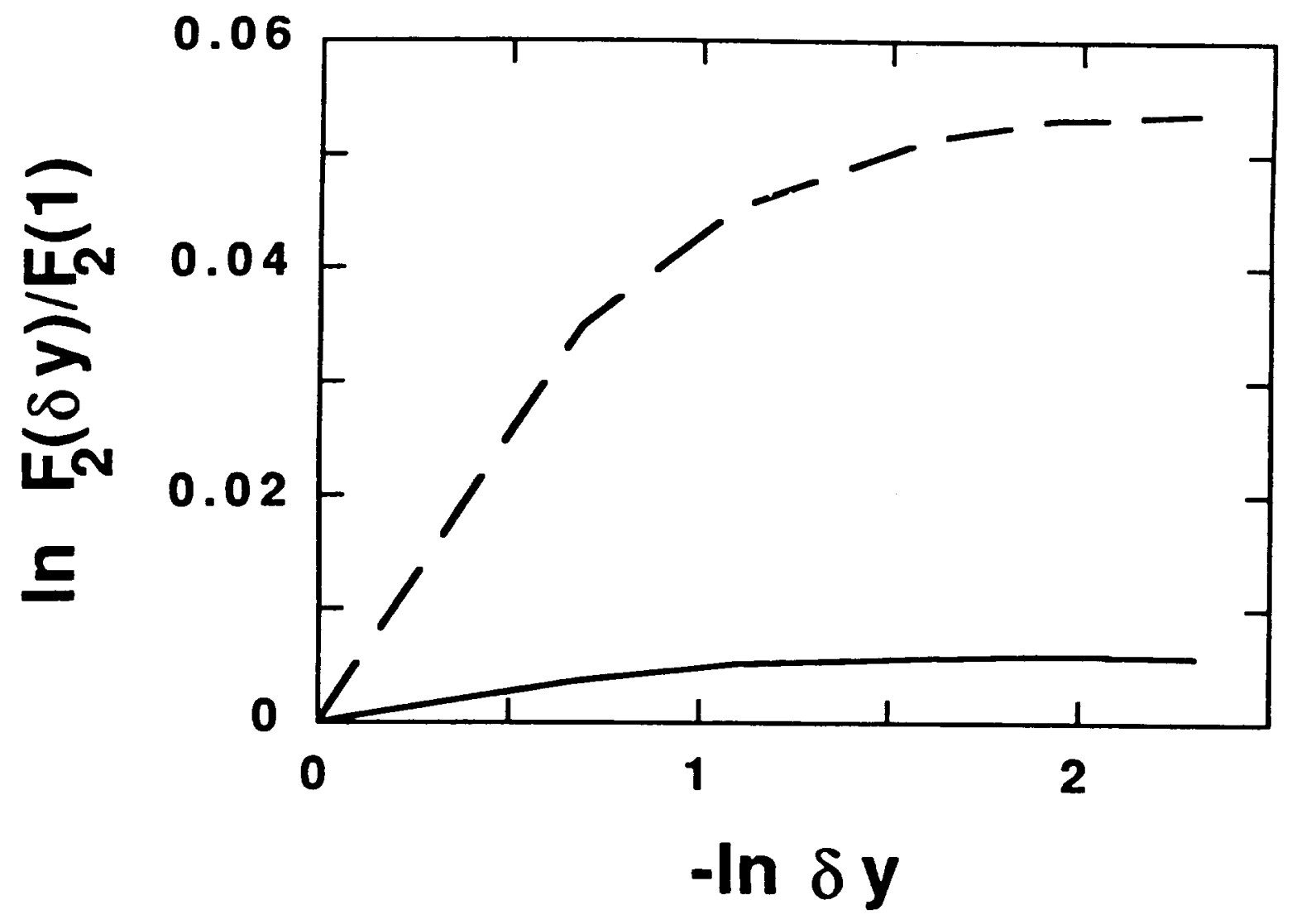

Fig. 17 


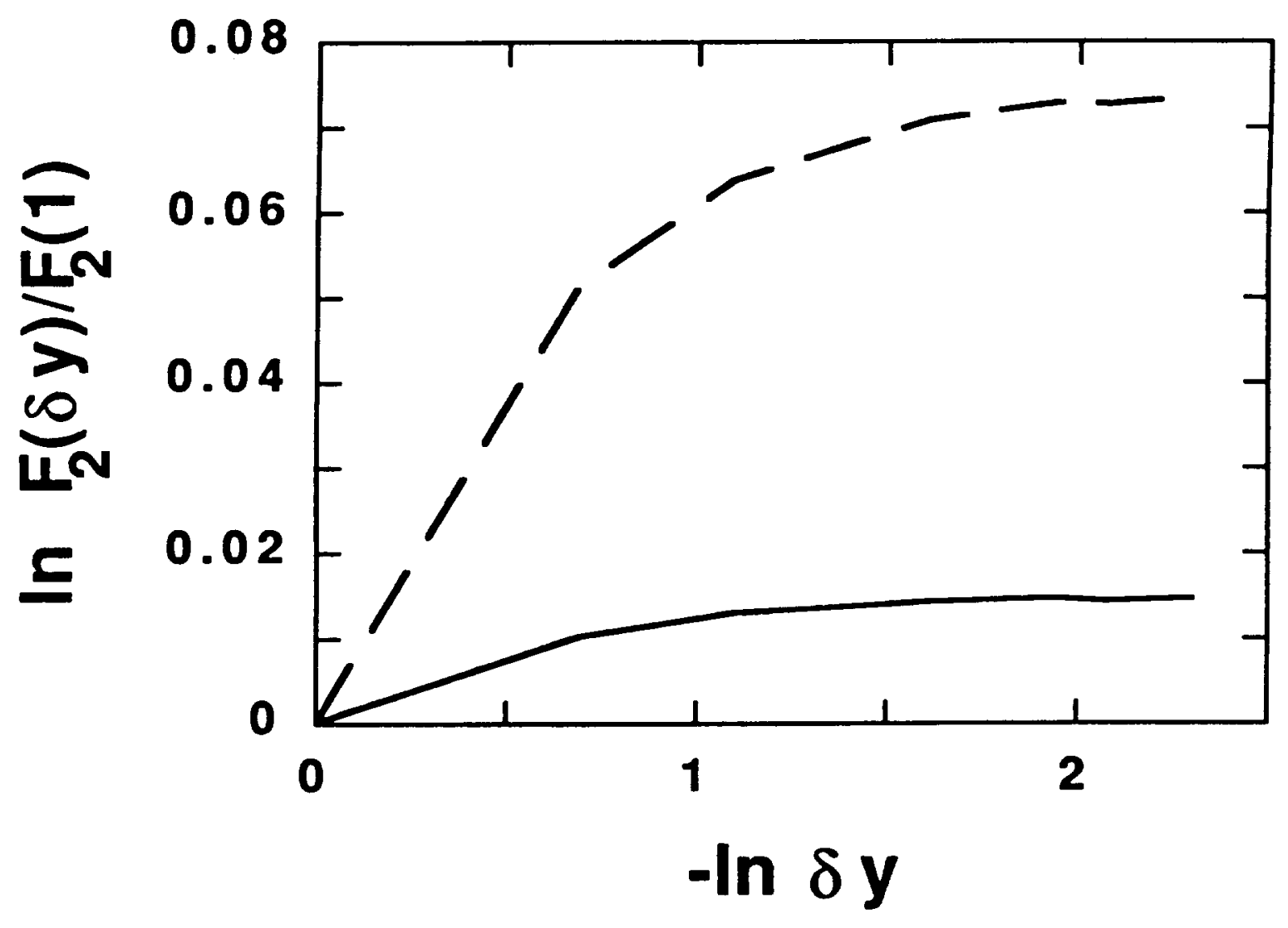

Fig. 18 


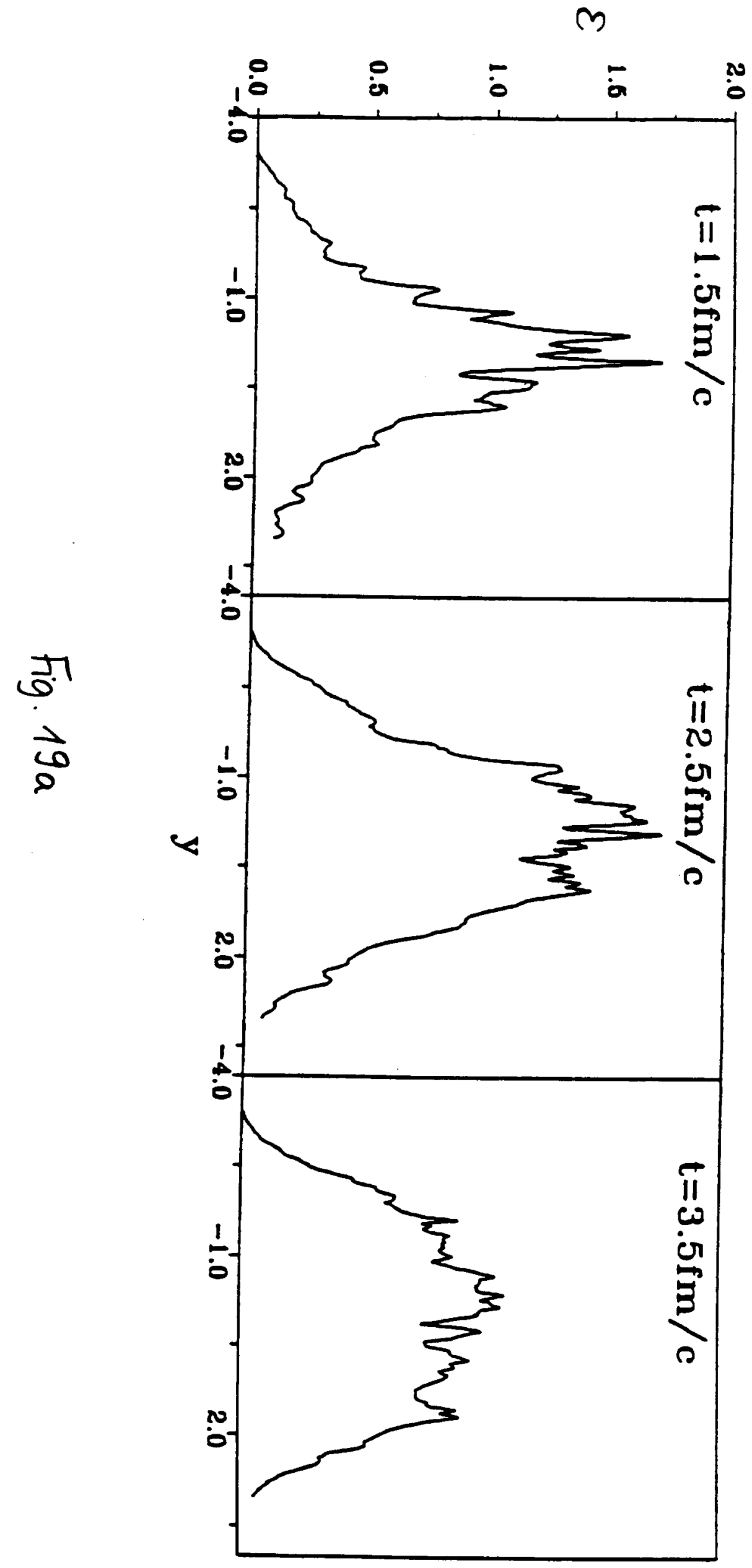




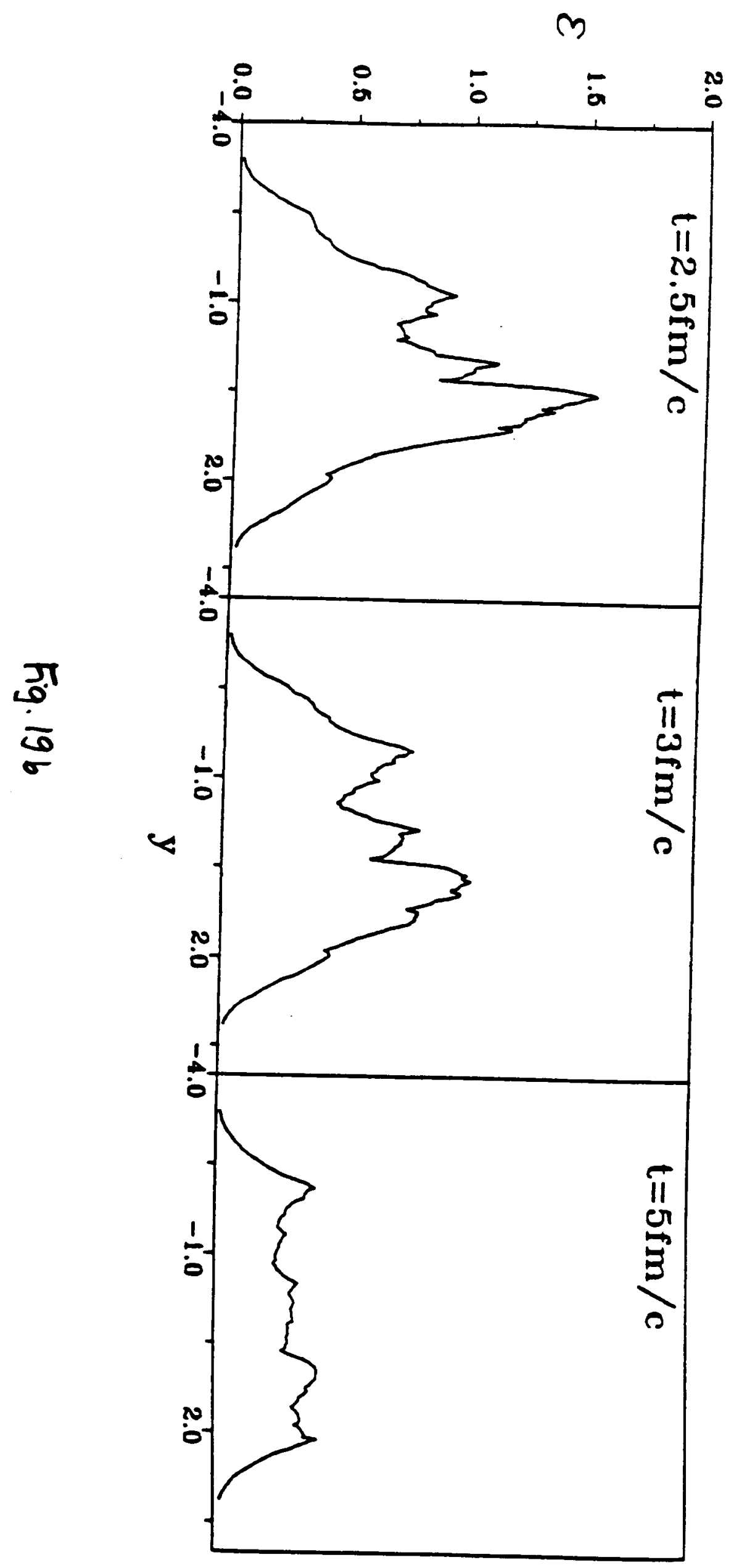




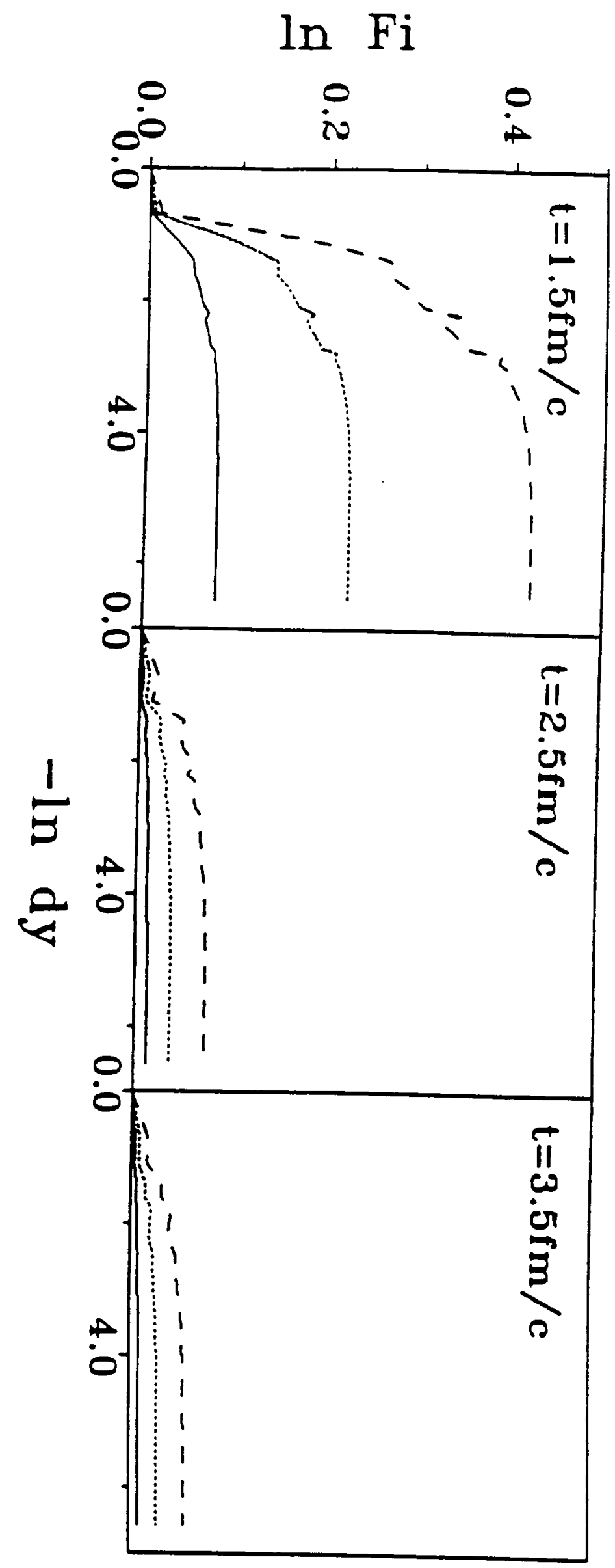

10

0 


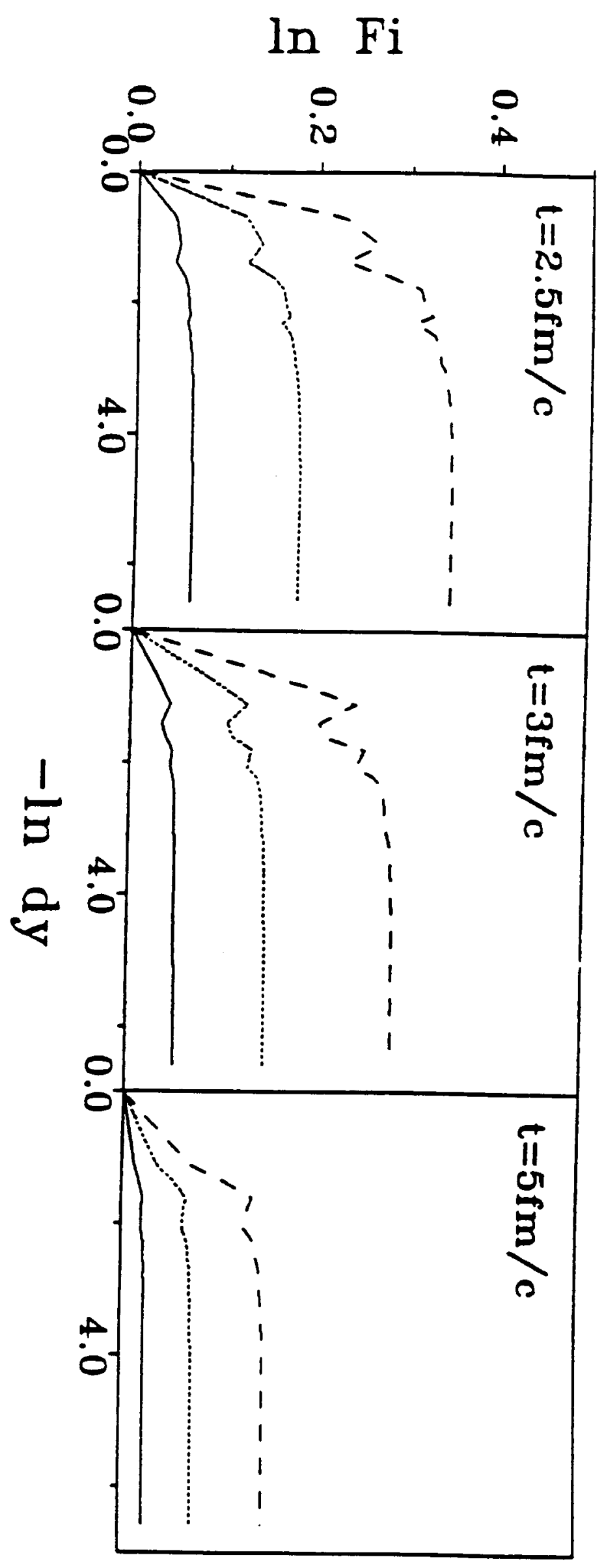

$T$
$\dot{0}$
0
0 
$\frac{\pi}{2}$

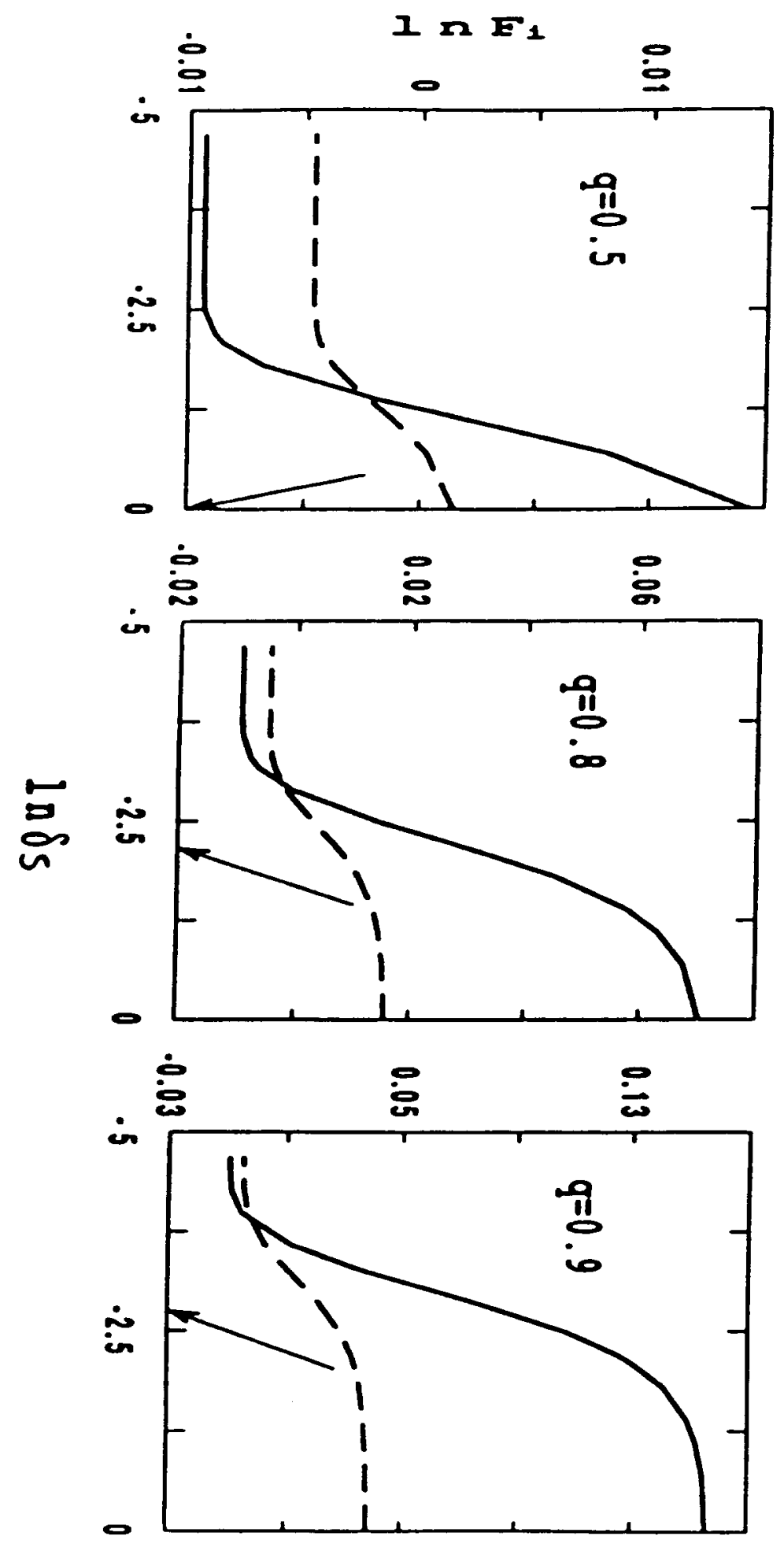




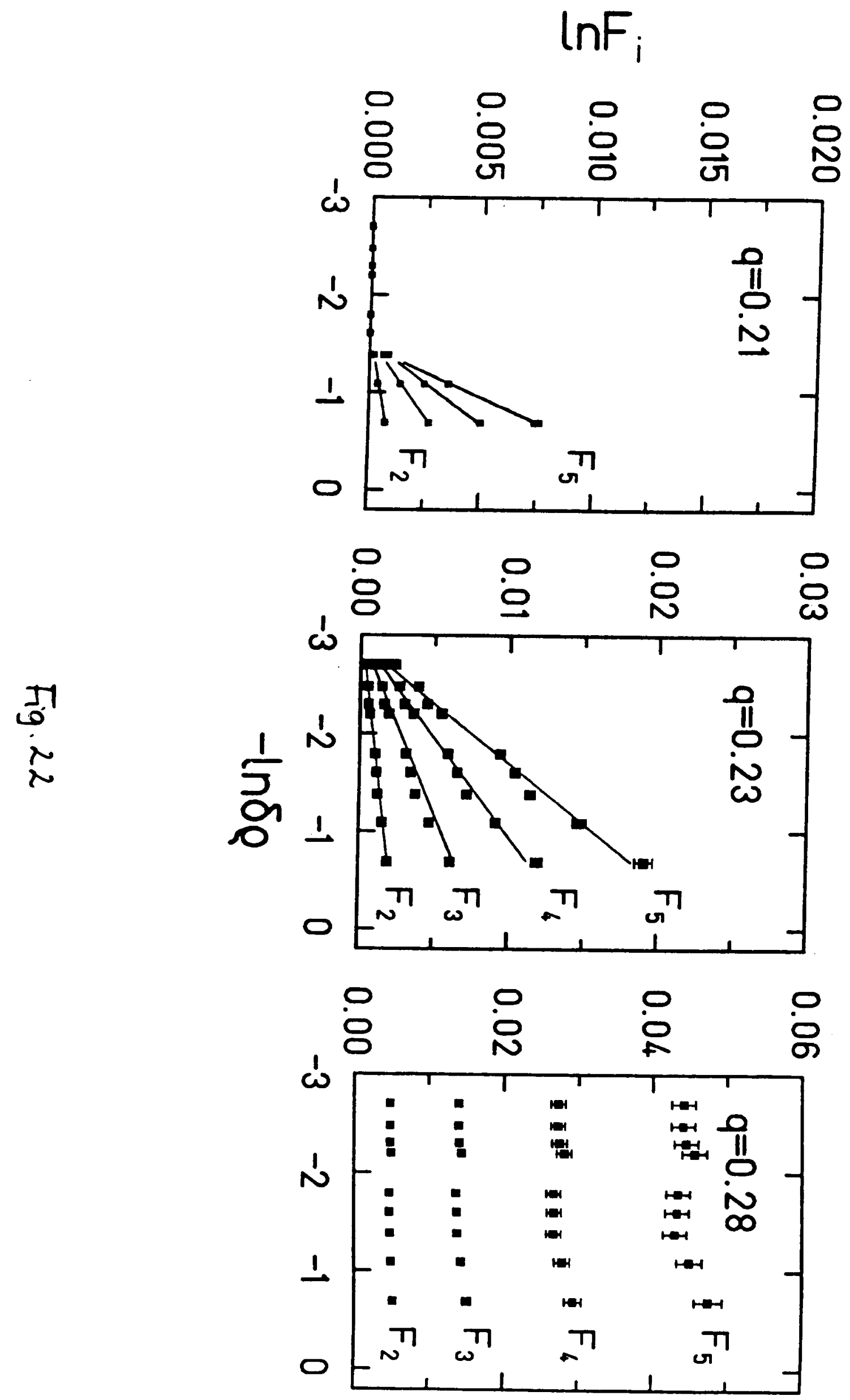


Experiment

Percolation Model
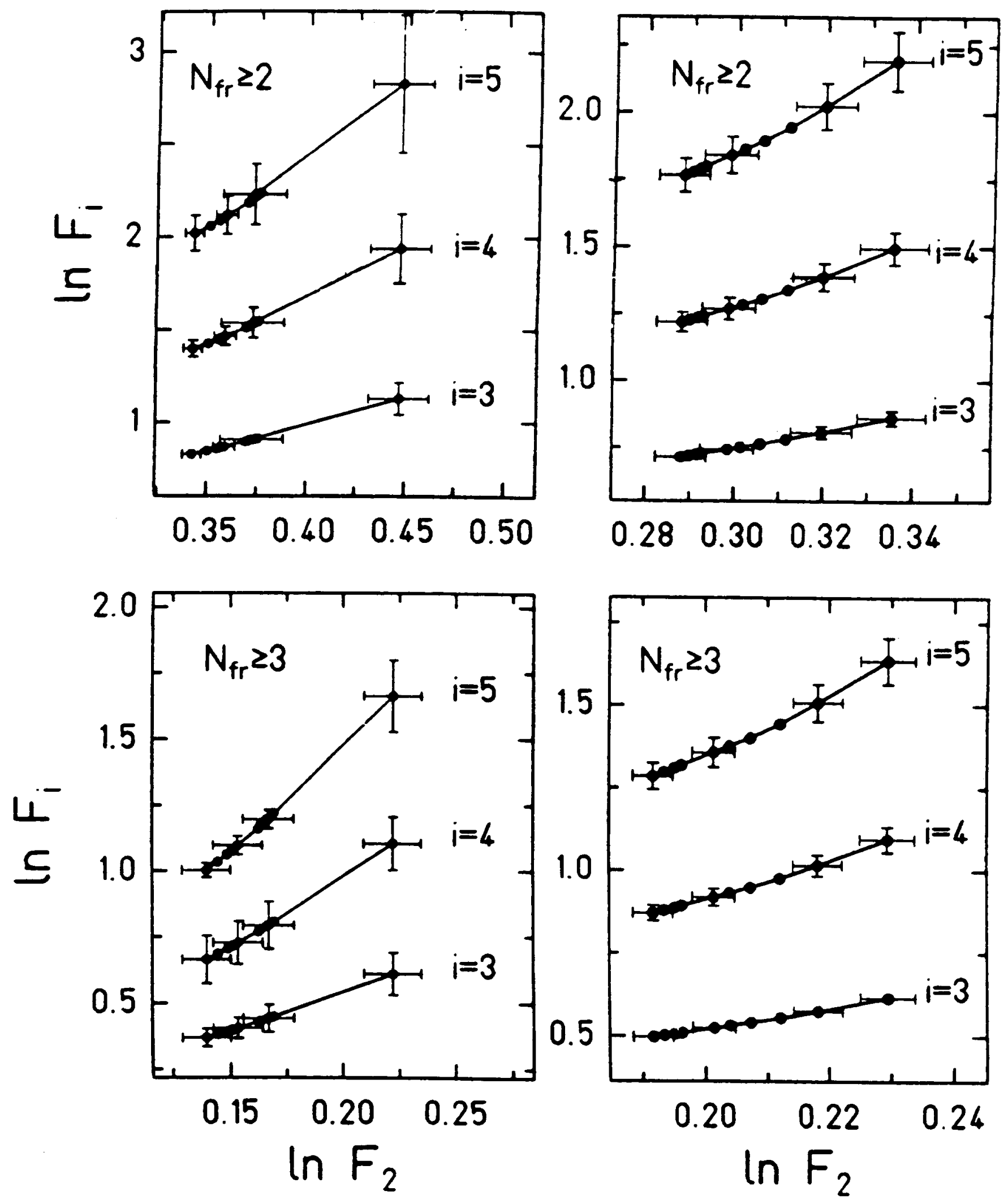


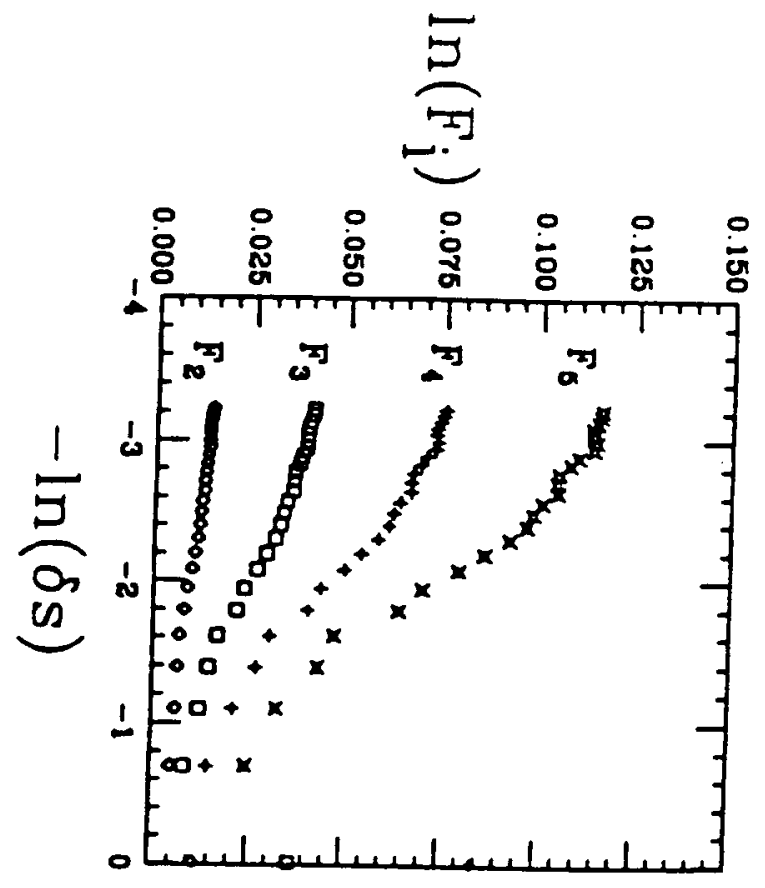

\section{z}
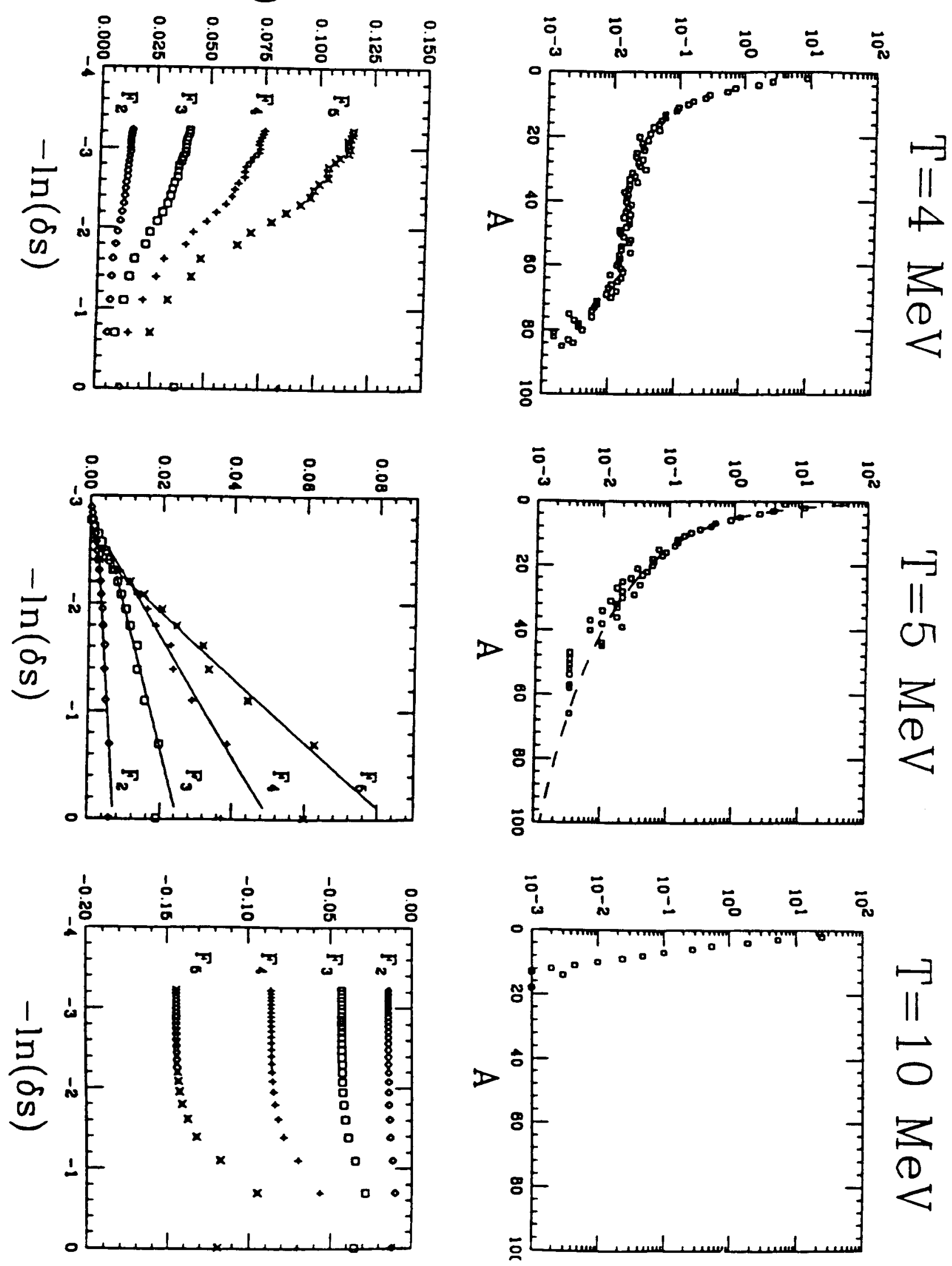


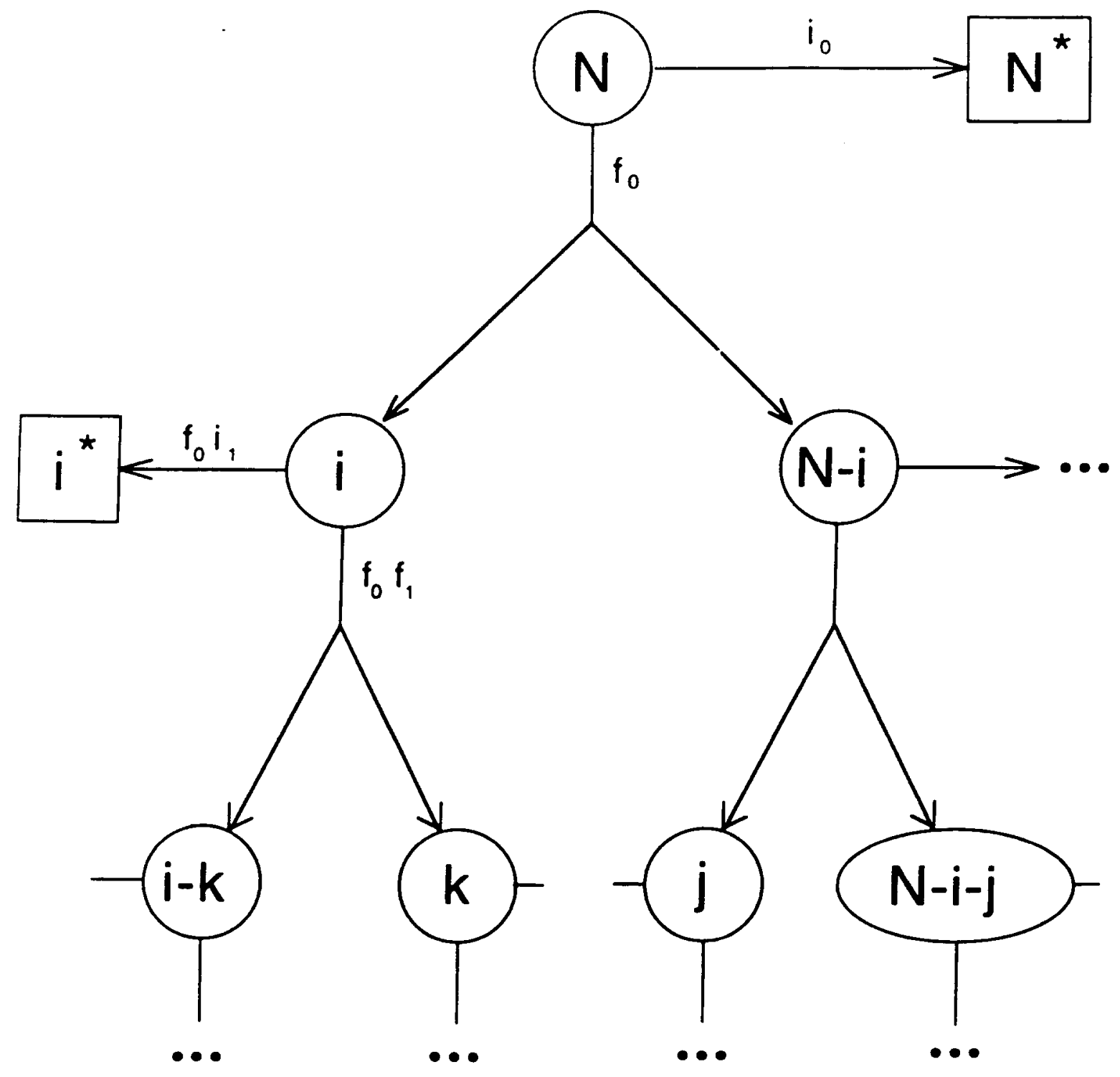

Fig. 25 


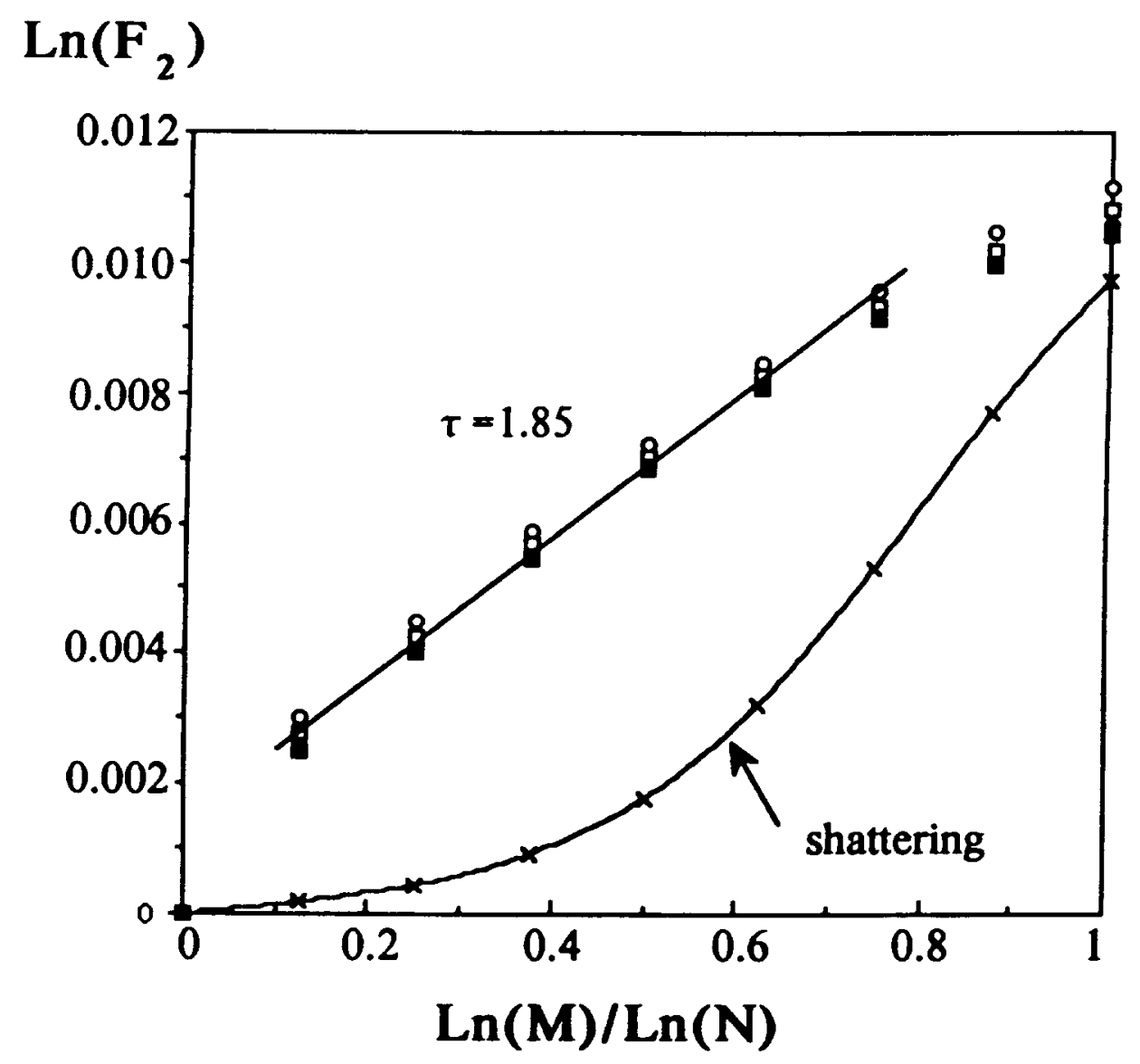

Fig. 26 


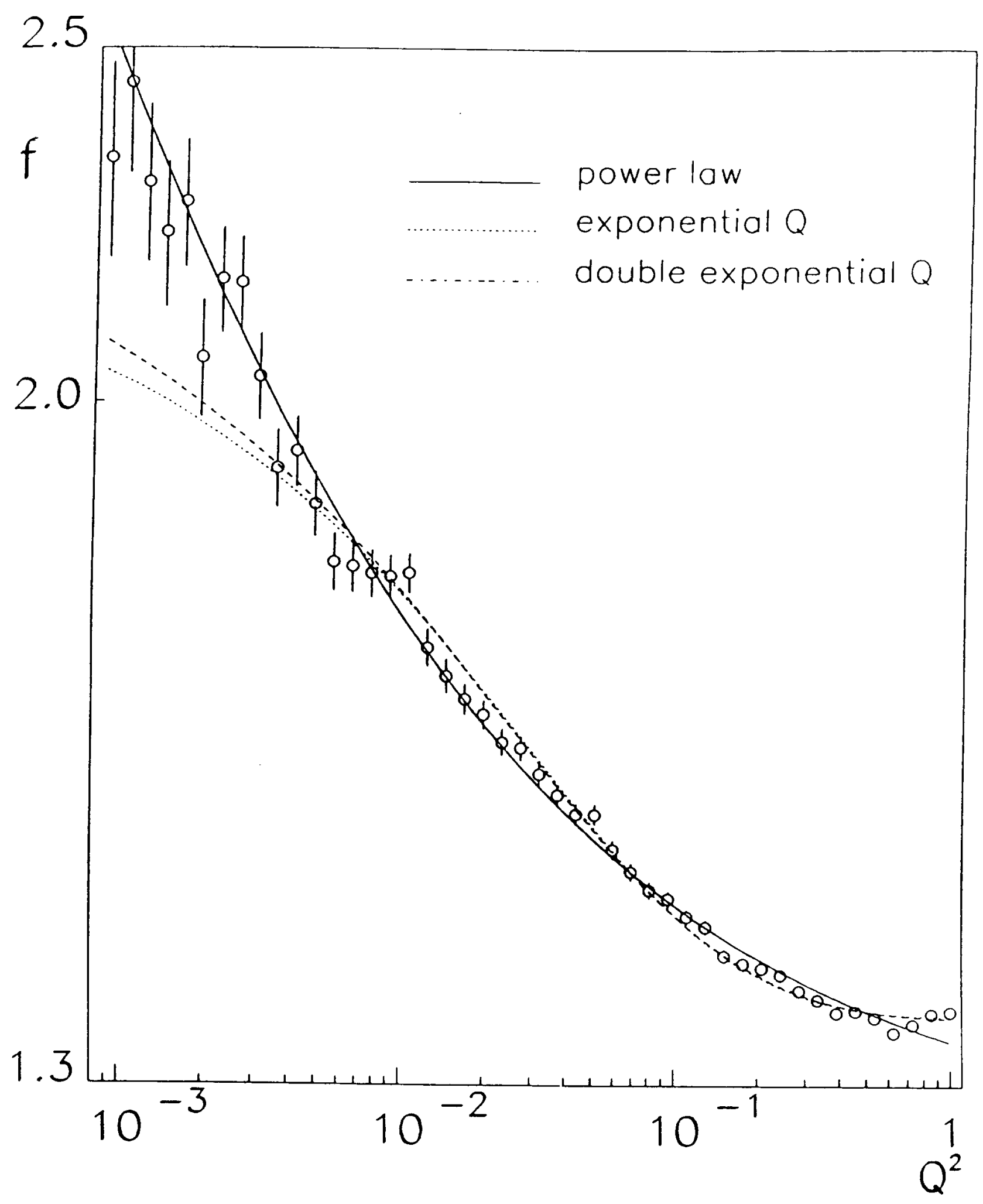

Fig. 27 


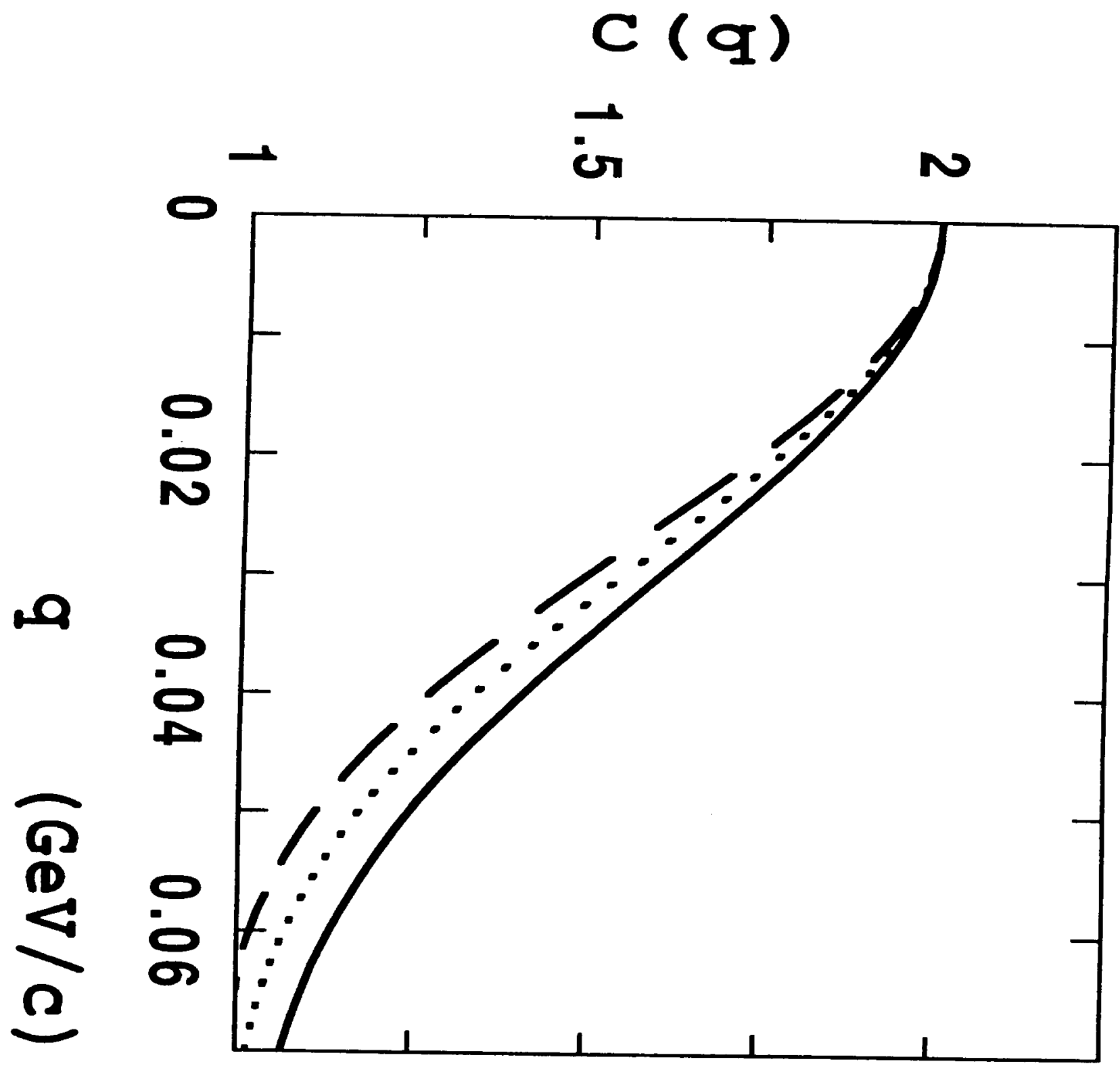

$\frac{\pi}{0}$
in
$\infty$ 


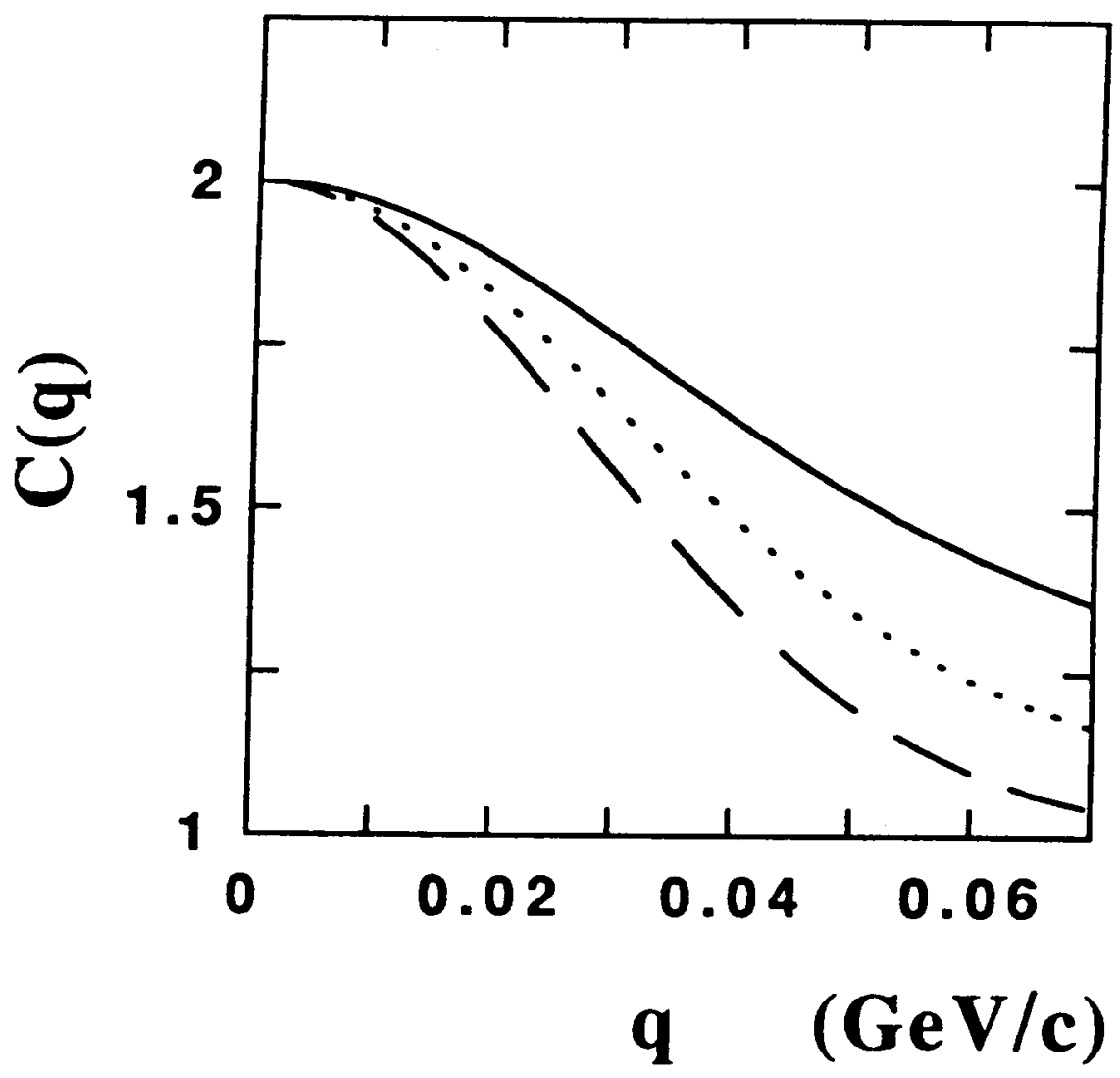

r. $a$ 